\title{
SYNERESIS AND RHEOLOGY MECHANISMS OF A LATEX-HEUR ASSOCIATIVE THICKENER SYSTEM
}

\author{
A Project Report \\ presented to \\ the Faculty of California Polytechnic State University, \\ San Luis Obispo
}

\author{
In Partial Fulfillment \\ of the Requirements for the Degree \\ Master of Polymers and Coatings Science
}

by

Travis Bruno Smith

December 2015 
(C) 2015

Travis Bruno Smith

ALL RIGHTS RESERVED 


\section{COMMITTEE MEMBERSHIP}

TITLE:

Syneresis and Rheology Mechanisms of a Latex-HEUR

Associative Thickener System
AUTHOR:
Travis Bruno Smith

DATE SUBMITTED: December 2015

COMMITTEE CHAIR: Raymond Fernando, Ph.D.

Professor of Polymers and Coatings

COMMITTEE MEMBER: Philip Costanzo, Ph.D.

Associate Professor of Polymers and Coatings

COMMITTEE MEMBER: Hasan Palandoken, Ph.D.

Associate Professor of Chemistry and Biochemistry 


\begin{abstract}
Syneresis and Rheology Mechanisms of a Latex-HEUR Associative Thickener System Travis Bruno Smith
\end{abstract}

Rheology modifiers are used in paints and coatings to ease their application to a surface, prevent sagging once applied, and allow the leveling of brushstrokes, among other benefits. The early rheology modifiers were hydroxyethyl celluloses (HECs), a type of non-associative thickener that is relatively inexpensive and synthesized from cellulose, which is abundant. However, coatings that are modified with HECs tend to suffer from poor leveling and syneresis (phase separation). HECs have since been replaced with associative thickeners (ATs). These thickeners, when properly formulated, produce stable dispersions that have improved rheological properties, yet, unlike HECs, are sensitive to changes to the coating formulation. This drawback has encouraged research that attempts to predict the phase behavior and rheology of systems that are modified with ATs.

This work is concerned with the phase behavior and rheology of waterborne latex / hydrophobically-modified ethoxylated urethane (HEUR) AT systems. When latex volume fraction is held constant, the amount of HEUR (and surfactant) in the mixture determines whether the system experiences syneresis. Dispersion phase diagrams (DPDs) of such systems have been previously studied, but the rheology of the mixtures used to prepare the DPDs have not been studied in any detail. A study on the rheology of phase separated latex / HEUR mixtures that were prepared with commercial materials was done at Cal Poly and showed a correlation between syneresis and complex rheology. However, 
a proper analysis was limited because the compositions and chemical structures of the commercial materials were not well known.

To better understand the relationships between phase behavior and rheology, waterborne latex / HEUR mixtures were prepared from latex and HEURs that were made at Cal Poly. Three series of mixtures were studied: commercial latex / commercial HEUR (I), commercial latex / Cal Poly HEUR (II), and Cal Poly latex / Cal Poly HEUR (III). The latex volume fraction was held constant at 0.25 and the concentration of HEUR was varied from 0-2.0 wt\%. Mixtures were allowed to equilibrate for 7 days, syneresis was measured on day 7, and steady-state viscosities over a shear rate range of $0.01-1000 \mathrm{~s}^{-1}$ were determined on days 7-9 with a DHR-2 rheometer (TA Instruments) that was outfitted with $40 \mathrm{~mm}, 2^{\circ}$ cone. The mixtures were also studied by microscopy and dynamic oscillatory testing. The chemical structures of the Cal Poly HEURs were determined by proton nuclear magnetic resonance spectroscopy $\left({ }^{1} \mathrm{H}\right.$ NMR $)$ and the molecular weight by gel permeation chromatography (GPC).

From this study, a correlation between syneresis and complex rheology was observed in I. Similar trends were observed in phase-separated II and dispersed (not phase-separated) III, though II with over $0.4 \mathrm{wt} \%$ HEUR were ejected from the cone / plate geometry at $1-100 \mathrm{~s}^{-1}$ and III did not demonstrate syneresis. Further investigation of dispersed II and phase-separated III is recommended to confirm the presence of the syneresis-rheology correlation of I in both II and III. In addition to these trends, only 2.1-4.2 wt\% II were able to be analyzed by the single-mode Maxwell model. Also the transition from phase-separated to stable dispersion was observed with a polarized microscopy at $5 x$ magnification. In conclusion this study represents progress in the 
ongoing study at Cal Poly to better understanding the mechanisms behind the syneresis and rheology of these latex / HEUR AT dispersions. 


\section{ACKNOWLEDGMENTS}

This project would not have happened without the support of the Arthur C. Edward Endowment, Cal Poly Bill More Fellowship Fund, and the facilities of Cal Poly's Kenneth N. Edwards Western Coatings Technology Center. There are several individuals who deserve special recognition for their valuable advice and support. My advisor, Dr. Raymond Fernando, got me started on this journey, imparted knowledge and expertise, and sparked my interest in the paints and coatings field. Dr. Philip Costanzo gave me tips on how to improve reaction setups and showed me how to use the GPC. Dr. Max Wills, though not directly involved in the project, showed me how to be creative with the tools and glassware that were at my disposal. Daisy Jauregui supplied the Cal Poly latex that was used in this work and Richard Morrison helped me better understand the mathematics behind the rheometer. 


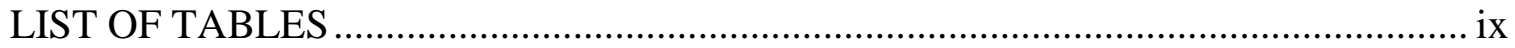

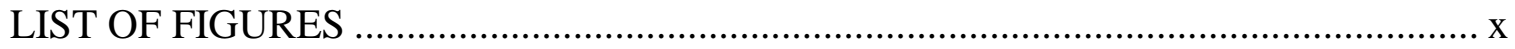

CHAPTER

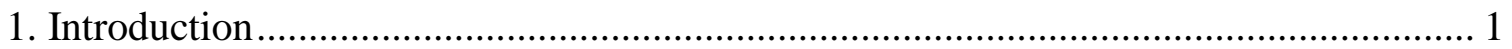

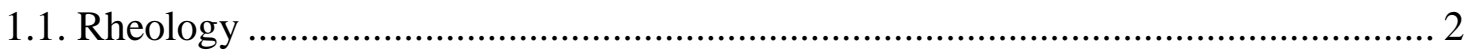

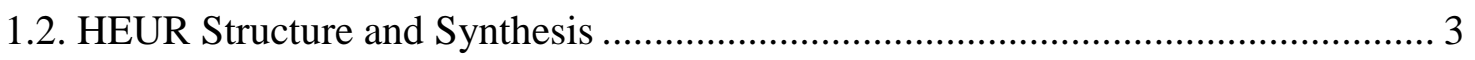

1.3. Phase Behavior and Rheology of Aqueous HEUR Mixtures.................................... 5

1.4. Rheology and Phase Behavior of Waterborne Latex / HEUR Mixtures................... 8

1.5. Impact of Surfactants and Cosolvents ............................................................. 10

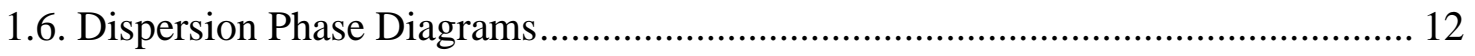

1.7. Modeling HEUR-based Systems with the Maxwell Model ................................... 13

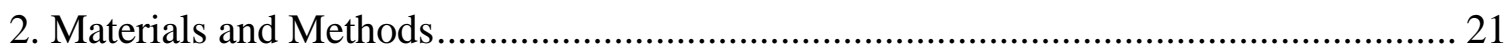

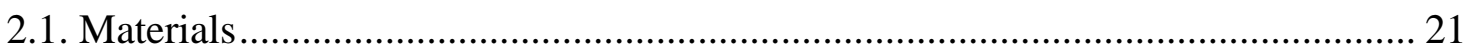



2.3. Preparation and Analysis of Waterborne Latex / HEUR Mixtures ........................ 26

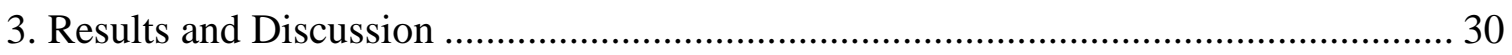

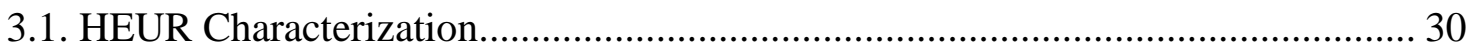

3.2. Comparison of Powder and Stock Sample Preparation Methods .......................... 34

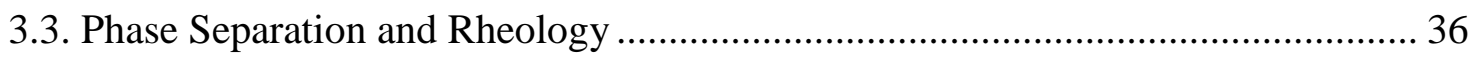

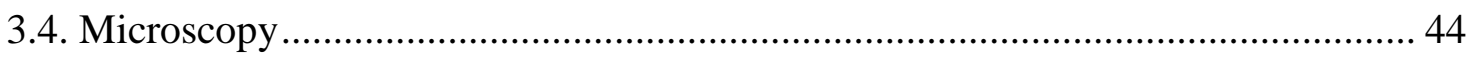

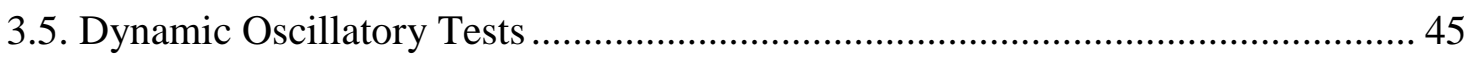

4. Conclusions and Future Work …………………................................................... 48

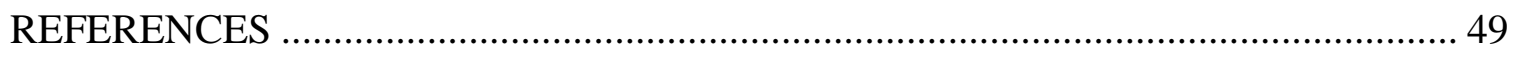

APPENDICES

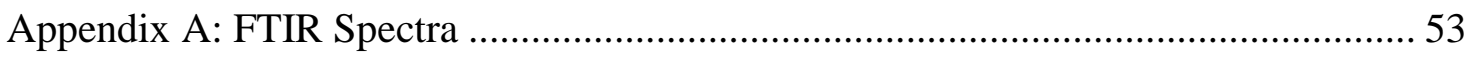

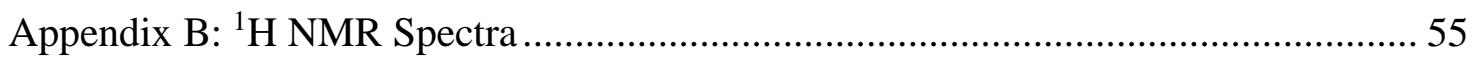

Appendix C: Waterborne Latex / HEUR Mixture Compositions ................................... 60

Appendix D: Determination of Percent Syneresis Using ImageJ ................................. 65 


\section{LIST OF TABLES}

Table

Page

Table 1. Solids Content, Particle Diameter, and Density of Commercial Latex, ${ }^{36}$ Commercial HEUR, ${ }^{37}$ and Cal Poly Latex, and Molecular Weight and Polydispersity Index of Commercial HEUR. The Molecular Weight of Commercial HEUR Was Determined by Gel Permeation Chromatography.

Table 2. General Compositions of Waterborne Latex / HEUR Mixtures.... 27

Table 3. Parameters for the Rheological Testing of Waterborne Latex / HEUR Mixtures. Mixtures Were Subject to a Flow Peak Hold and then a Flow Peak Hold....... 28

Table 4. Parameters for the Dynamic Oscillatory Testing of Waterborne Latex / HEUR Mixtures. Mixtures Were Subject to a Strain Sweep and Then a Frequency Sweep.

Table 5. Molecular Weight, Polydispersity Index, and Composition of Cal Poly HEUR. The Molecular Weight and PDI Were Obtained by GPC and the Composition and Extent of Reaction by ${ }^{1} \mathrm{H}$ NMR.

Table 6. Commercial Latex / Commercial HEUR (I), Commercial Latex / Cal Poly HEUR (II), and Cal Poly Latex / Cal Poly HEUR Mixtures (III) That Showed Syneresis. The Concentrations of Mixtures that Showed Syneresis Are Listed in The Table.

Table 7. Variables That Are Used in Eqs 16-35 to Calculate the Compositions of Waterborne Latex / HEUR Mixtures.

Table 8. Compositions of the Commercial Latex / Commercial HEUR Mixtures.

Table 9. Compositions of the Commercial Latex / Cal Poly HEUR Mixtures. 63

Table 10. Compositions of the Cal Poly Latex / Cal Poly HEUR Mixtures. 64

Table 11. Error of the Calculated Percent Syneresis Values (as a Percent) of the Waterborne Latex / HEUR Mixtures as the Size of the Top and Bottom Layers Varies. 


\section{LIST OF FIGURES}

Figure

Page

Figure 1. Synthesis of uni-HEUR from Poly(ethylene glycol) and Monoisocyanate......... 4

Figure 2. Synthesis of SG-HEUR from Poly(ethylene glycol) and Diisocyanate,

Followed By End-Capping with Alkyl Alcohol. 4

Figure 3. Typical Structure of uni-HEUR $(m=0)$ and SG-HEUR $(m>1) \ldots \ldots \ldots \ldots \ldots \ldots . . .5$

Figure 4. HEUR Micellar Structures as a Function of HEUR Concentration. ................... 6

Figure 5. Effect of Shear Rate on the Structure of the HEUR Associative Network. ....... 7

Figure 6. Schematic of Latex-HEUR Interactions. .............................................. 9

Figure 7. Effect of Surfactant Concentration on HEUR Solution Viscosity. .................. 11

Figure 8. Dispersion Phase Diagrams of Latex / HEUR / Surfactant Mixtures. .............. 13

Figure 9. Maxwell Model at Rest (a) and Responding Elastically (b) or Viscously (c)... 14

Figure 10. Zero-shear Viscosity of 2.1-4.2 wt\% Commercial Latex / Cal Poly HEUR (Samples 96-99) as a Function of HEUR Concentration. ............................................ 15

Figure 11. Sinusoidal Stress of System at 0, 90, and 45 Degree Phase Angles............... 17

Figure 12. Strain Sweep, Whereby Strain Varied and Frequency is Held Constant. ....... 17

Figure 13. Frequency Sweep, Whereby Frequency Varied and Strain is Held Constant.

Figure 14. Strain Sweep (with Linear Viscoelastic Region) of $0.51 \mathrm{wt} \%$ Commercial Latex / Commercial HEUR Mixture......................................................................... 18

Figure 15. Cole-Cole Plot of 4.2 wt\% Commercial Latex / Commercial HEUR Mixture, Which Does Fit the Single-Mode Maxwell Model.......................................... 19

Figure 16. Synthesis of Cal Poly HEUR from PEG20 and Octadecyl Isocyanate .......... 23

Figure 17. Synthesis of Urea from Isocyanate and Water (Top), Biuret from Urea Above $100{ }^{\circ} \mathrm{C}$ (Middle), and Allophanate from the Carbamate Group of HEUR Above $100{ }^{\circ} \mathrm{C}$ (Bottom). 23 
Figure 18. (A) Chemical Structures of uni-HEUR (Top) and TFAA End-capped PEG20 (Bottom); (B) ${ }^{1} \mathrm{H}$ NMR of TS-I-036; (C) ${ }^{1} \mathrm{H}$ NMR of TS-I-036 That Was Treated With TFAA.

Figure 19. GPC Chromatographs of Commercial HEUR (Top) and Cal Poly HEUR (Bottom).

Figure 20. Viscous Top Layer in Commercial Latex / Cal Poly HEUR (Left), and Cal Poly Latex / Cal Poly HEUR Mixtures (Right) on Day 7.

Figure 21. Clear Top Layer in Commercial Latex / Cal Poly HEUR Mixtures on Day 7

Figure 22. Percent Syneresis in Commercial Latex / Commercial HEUR Mixtures on Day 7. After Rheological Testing Had Ended, Samples Were Allowed to Remain Undisturbed and Percent Syneresis Was Measured After 9 and 21 Days at Rest.

Figure 23. Percent Syneresis in Commercial Latex / Cal Poly HEUR Mixtures on Day 7. After Rheological Testing Had Ended, Samples Were Allowed to Remain Undisturbed and Percent Syneresis Was Measured After 7 and 14 Days at Rest. Samples Were Subject to a Second Round of Rheological Testing, Were Allowed to Remain Undisturbed for 21 Days, and Percent Syneresis Was Measured on that $21^{\text {st }}$ Day.

Figure 24. Viscosity Profiles of Commercial Latex / Commercial HEUR (Day 11). The Volume Fraction of Latex Was 0.25 and the Concentrations of HEUR Are Provided Inside the Figure.

Figure 25. Viscosity Profiles of Commercial Latex / Cal Poly HEUR (Day 7). The Volume Fraction of Latex Was 0.25 and the Concentrations of HEUR Are Provided Inside the Figure.

Figure 26. Ejection of Commercial Latex / Cal Poly HEUR Mixture Samples from the Cone / Plate Geometry At Within 1-100 s ${ }^{-1}$.

Figure 27. Viscosity Profiles of Cal Poly Latex / Cal Poly HEUR (Day 54). The Volume Fraction of Latex Was 0.30 and the Concentrations of HEUR Are Provided Inside the Figure.

Figure 28. Transition from Irregular to Featureless Appearance in 0.10, 1.83, 2.12, and $3.19 \mathrm{wt} \%$ Commercial Latex / Cal Poly HEUR Mixtures at 5x Magnification. The Black Circular Shapes in the Photographs of 1.83 and $2.12 \mathrm{wt} \%$ HEUR Are Air Bubbles That Were Introduced During Sample Mixing. 
Figure 29. (A) Cole-Cole Plot of 4.2 wt\% Commercial Latex / Cal Poly HEUR, Which Fits the Single-Mode Maxwell Model; (B) Cole-Cole Plot of 1.83 wt $\%$ Commercial Latex / Cal Poly HEUR, Which Does Not Fit the Single-Mode Maxwell Model.

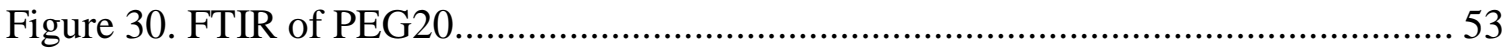

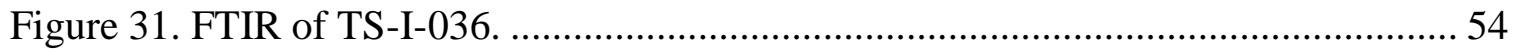

Figure 32. Chemical Structures of TS-I-036 (Top) and PEG20 That Was Treated With TFAA (Bottom). The Labelled Protons Correspond to the Assigned Peaks in Figures 33-36. 55

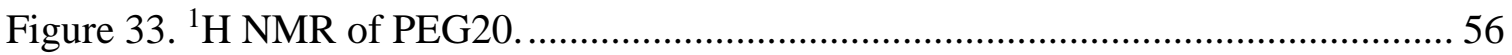

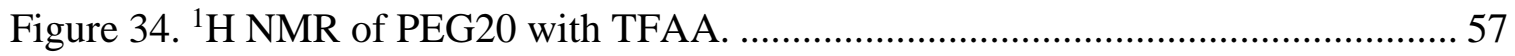

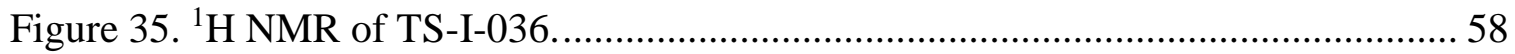

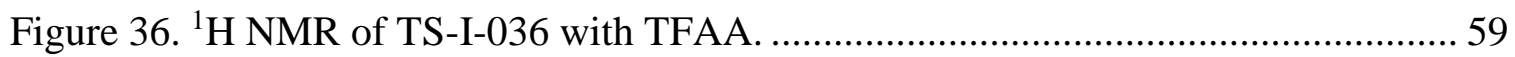

Figure 37. Yellow-line Drawn Across the Sample 73 (Day 32) using ImageJ................ 67

Figure 38. Plot Profile of Sample 73 (day 32), Obtained with ImageJ. The Arrows Correspond to the Pixel Values that Mark the Boundaries of the Top and Bottom Layers of the Waterborne Latex / HEUR Mixture. 68 


\section{Introduction}

Rheology modifiers are used in paints and coatings to ease their application to a surface, prevent sagging once applied, and allow the leveling of brushstrokes, among other benefits. ${ }^{1,2}$ Hydroxyethyl celluloses (HECs), a non-associative thickener, are a rheology modifier that was traditionally used in paints and coatings. ${ }^{2}$ However, coatings modified by HECs tend to suffer from poor leveling and syneresis (phase separation). ${ }^{3}$ In high quality paints (e.g. semi-gloss paints), they have since been replaced with associative thickeners (ATs), which produce stable dispersions that have improved rheological properties. ${ }^{1,4}$ These benefits are possible because ATs interact with the components of the coating, including other AT molecules. ${ }^{1}$ This advantageous property also makes ATs highly sensitive to changes to the coating formulation and prolongs the development of novel coatings that are modified with ATs. ${ }^{1}$

The need to predict how ATs behave in a formulation has encouraged significant research over the past decades. This work is concerned with the phase behavior and rheology of waterborne latex / hydrophobically-modified ethoxylated urethane (HEUR) AT mixtures. While the phase behavior and rheology of HEURs have been extensively studied, not many studies have attempted to correlate the phase behavior to rheology. At Cal Poly, a correlation between syneresis and complex rheology in waterborne latex / HEUR mixtures that were prepared from commercial materials has been observed. ${ }^{5}$ When syneresis was absent, complex rheology was not observed. ${ }^{5}$ However, a proper analysis was limited because the compositions and chemical structures of the commercial materials were not well known. ${ }^{5}$ To better understand the relationships between the phase behavior and rheology of these systems, waterborne latex / HEUR mixtures were 
prepared from materials that were made at Cal Poly. A study such as this represents progress in the ongoing study to better understanding the mechanisms behind the syneresis and rheology of these simple latex / HEUR AT thickeners systems. Yet, before the results of this study are shared, several trends regarding the phase behavior and rheology of simple HEUR-based systems will be discussed.

\subsection{Rheology}

Rheology is the study of the flow and deformation of fluids, such as paints and coatings. The fluid's viscosity $\eta$ measures the resistance to flow and is determined from the ratio of the shear stress $\sigma$ and shear rate $\dot{\gamma}$ (eq 1). ${ }^{6}$ The shear stress is related to the force that is applied to the fluid to induce flow and the shear rate is the velocity gradient across the fluid. ${ }^{6}$

$$
\eta=\frac{\sigma}{\dot{\gamma}}
$$

The shear-induced arrangement of fluid and particles within the fluid determine whether the viscosity will depend on the shear rate..$^{7-8}$ If the overall particle arrangement does not change, the viscosity also does not change and the flow is Newtonian. ${ }^{7-8}$ If the arrangement becomes more ordered, particles can flow more readily, the viscosity decreases with increasing shear rate, and the flow is shear thinning. ${ }^{7-8}$ When the arrangement becomes more disordered, the viscosity instead increases and the flow is shear thickening. ${ }^{7-8}$ 
At low shear rates (under $1 \mathrm{~s}^{-1}$ ), Brownian (thermal) motion, hydrodynamic volume, chain entanglements, and intermolecular associations (including flocculation, aggregation, and adsorption) contribute to the fluid's viscosity. ${ }^{7-8}$ At high shear rates (over $1000 \mathrm{~s}^{-1}$ ), the input of energy is sufficient to disrupt weak intermolecular associations, reverse chain entanglements, and overcome Brownian motion. ${ }^{7-8}$

\subsection{HEUR Structure and Synthesis}

HEURs are telechelic polymers that comprise a poly(ethylene glycol) (PEG) backbone that is terminated by short (C6-18) alkyl chains. ${ }^{9,10-18}$ They can be prepared by one of two methods (Figure 1-2). HEURs can be prepared from the direct addition of monoisocyanate to PEG, in which case it is called a uni-HEUR. ${ }^{9}$ HEURs can also be prepared from the step-growth (SG) polymerization of PEG and diisocyanate. ${ }^{9}$ Excess diisocyanate is used to control the molecular weight and to generate an isocyanatefunctionalized polymer that is then exposed to alkyl-amine or -alcohol. ${ }^{9,10-18}$ The resultant polymer is called a SG-HEUR. ${ }^{9}$ Unlike uni-HEURs, the hydrophilic segment of SGHEURs consists of multiple PEG polymers that are separated by diisocyanate segments. ${ }^{9}$ A schematic of uni- and SG-HEURs is provided in Figure 3.

The terminal alkyl segments are called external hydrophobes; their associations are responsible for the viscosifying behavior of HEURs. ${ }^{9,10-18}$ Holding molecular weight and concentration constant, the viscosities of aqueous HEUR solutions are several orders of magnitude greater than those of aqueous PEG solutions. This observation alone indicates that the presence of the external hydrophobes are responsible for the viscosifying behavior of HEURs. 
The diisocyanate segments of SG-HEURs are referred to as internal

hydrophobes $;^{9,10-18}$ they do not significantly contribute to the viscosity, as was shown in a fluorescence study of aqueous SG-HEUR solutions. ${ }^{18}$ That study used 8-

anilinonaphthalenesulfonic acid (ANS) fluorescent probe, whose peak emission intensity increases as the local environment becomes more nonpolar. ${ }^{18}$ The signal intensity of ANS increased dramatically as SG-HEUR concentration increased and only marginally when SG-HEUR that contained internal hydrophobes and no external hydrophobes was employed. ${ }^{18}$ This showed that internal hydrophobes do not significantly contribute to the viscosity of HEUR systems. ${ }^{18}$ As such, internal hydrophobes have been omitted from Figures 4-7.<smiles>[R]NC(=O)OCC(C)(C)COC(C)(C)COC(=O)N[R]</smiles>

Figure 1. Synthesis of uni-HEUR from Poly(ethylene glycol) and Monoisocyanate.

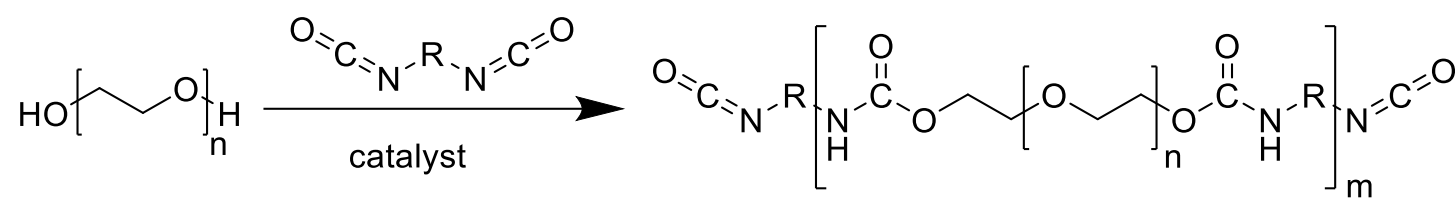

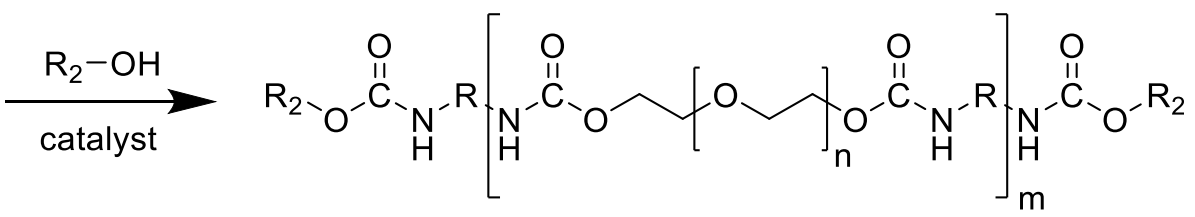

SG-HEUR

Figure 2. Synthesis of SG-HEUR from Poly(ethylene glycol) and Diisocyanate, Followed By End-Capping with Alkyl Alcohol. 


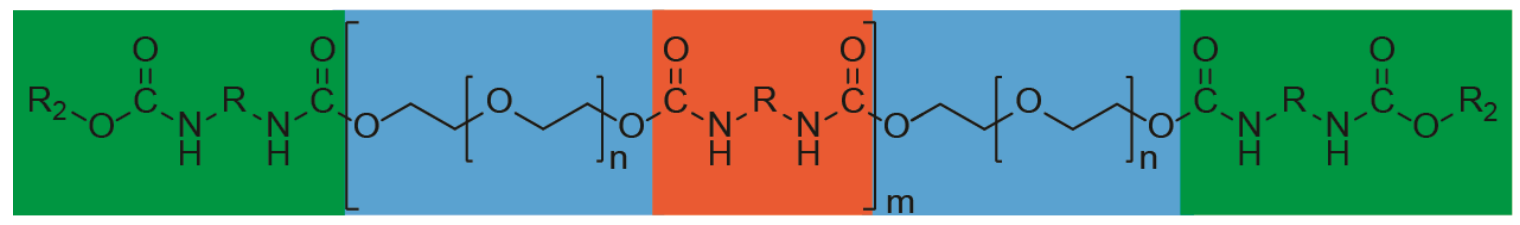

EXTERNAL HYDROPHOBE

HYDROPHILIC SEGMENT INTERNAL HYDROPHOBE HYDROPHILIC SEGMENT

EXTERNAL HYDROPHOBE

Figure 3. Typical Structure of uni-HEUR $(m=0)$ and SG-HEUR $(m>1)$.

\subsection{Phase Behavior and Rheology of Aqueous HEUR Mixtures}

The model that is used to correlate the chemical structures of HEURs to their rheology is the transient network model. The model was originally developed by Green and Tobolsky in 1946 and has since been refined by Lodge, Yamamoto, Baxandall, Annable, Semenov, Edwards, Tanaka, and many others. ${ }^{19-20}$ The model has also been used to elucidate the mechanisms that are responsible for the rheology of waterborne latex / HEUR / surfactant mixtures. In this and the following sections, a qualitative description of the rheology of simple HEUR-based systems will be explored using the transient network model. Only HEURs with molecular weights below the chain entanglement molecular weight will be considered. ${ }^{19}$

While in water, the HEUR's external hydrophobes can interact intramolecularly, which causes the HEUR to adopt a loop-like structure ${ }^{14,21}$ (Figure 4). At concentrations above the critical micellar concentration (CMC), the external hydrophobes can also interact intermolecularly, which forms flower-like micelles ${ }^{21-23}$ (Figure 4). As HEUR concentration increases, additional micelles (of similar size) are formed until micelles are close enough that the external hydrophobes can reside in two different micelles, which forms bridges between micelles. ${ }^{14,21-22}$ At all concentrations, the hydrophobic associations between external hydrophobes are reversible; external hydrophobes 
constantly enter and exit micelles via Brownian motion. ${ }^{24}$ When not in a micelle or loop, the external hydrophobes "dangle" in the continuous phase. ${ }^{23}$

When the concentration of HEUR exceeds the critical aggregation concentration (CAC), a dynamic three-dimensional associative network is formed ${ }^{13-14,21,23}$ (Figure 4). This marks a significant rheological transition; a Newtonian system (whose low viscosity is determined by the volume fraction of HEUR) is transformed into a viscous system that expresses Newtonian, shear thickening, and shear thinning behaviors at low, medium, and high shear rates, respectively ${ }^{9,10-18}$ (Figure 5).

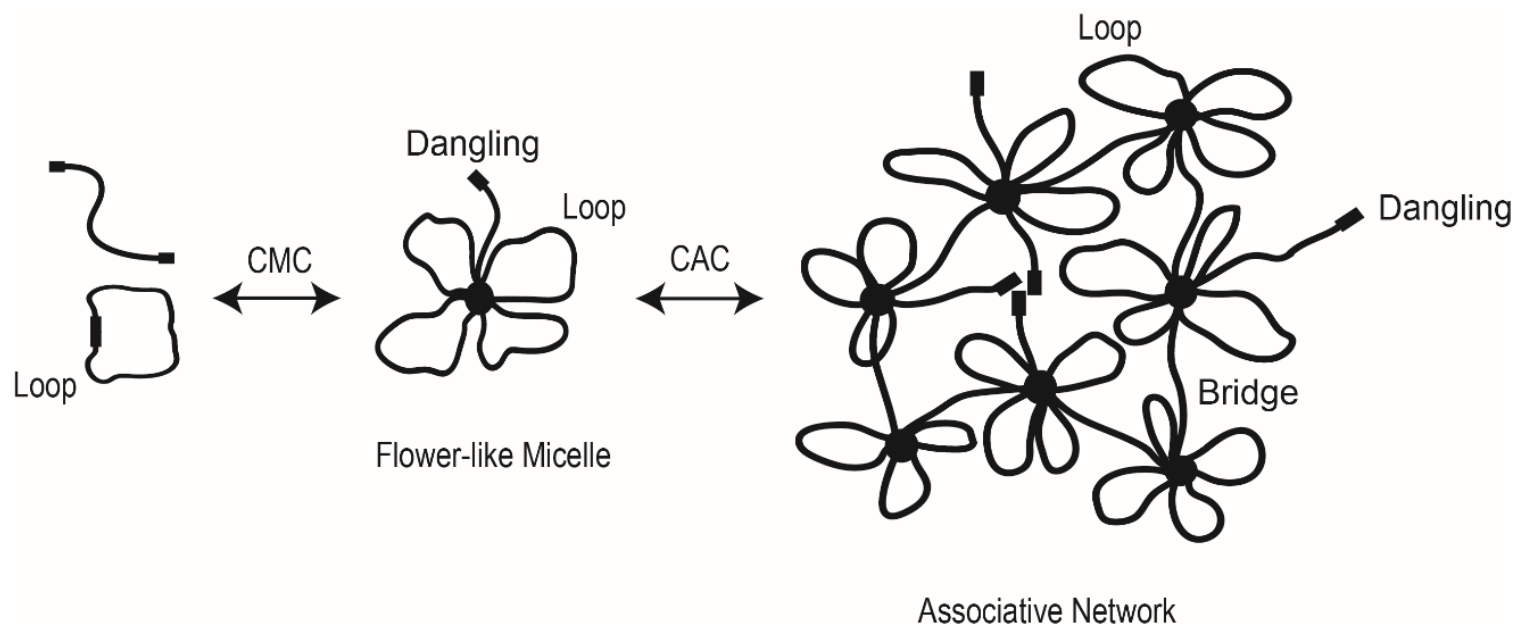

Figure 4. HEUR Micellar Structures as a Function of HEUR Concentration.

The dramatic change in rheology is due to the propagation of stress through the associative network. ${ }^{23}$ HEUR bridges can propagate stress whereas HEURs in loop and dangling configurations cannot. ${ }^{23}$ When stress is applied, bridges are extended, contracted, formed, broken, and reformed..$^{8,23,24}$ The system remains as disordered as before and shows Newtonian behavior ${ }^{8,23,24}$ (Figure 5). As additional stress is applied, the 
hydrophobes of stretched bridges exit micelles and assume loop or dangling configurations. ${ }^{22-23,25}$ This "bridge to loop" transition decreases the number of available pathways for the propagation of stress and facilitates the shear-induced ordering of micelles, which causes the viscosity to decrease with increasing shear rate. ${ }^{8,22-23,25}$ Yet, when the associative network is not fully developed, shear-induced reorientation of the micelles can increase the number of intermolecular associations. ${ }^{8,15,25-26}$ This increases viscosity over a short range of shear rates $\left(1-100 \mathrm{~s}^{-1}\right)$ before the onset of shear thinning at higher shear rates. ${ }^{8,15,25-26}$

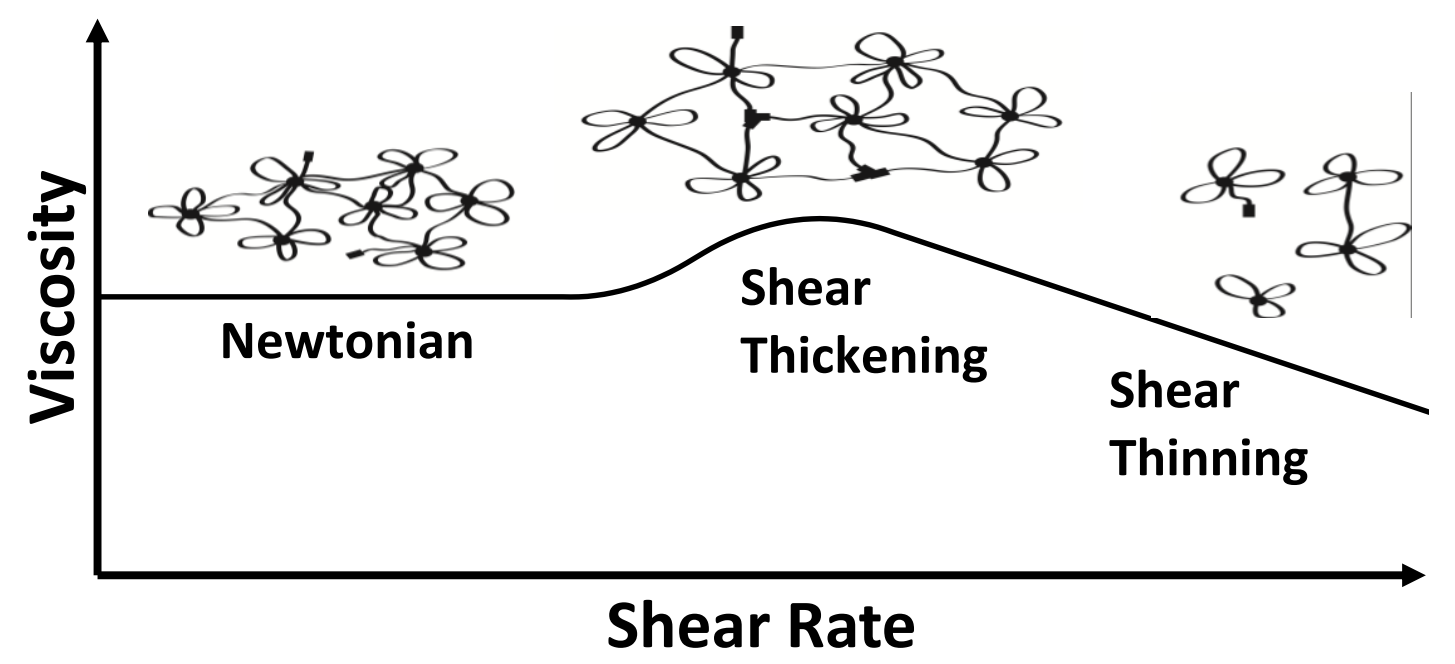

Figure 5. Effect of Shear Rate on the Structure of the HEUR Associative Network.

The length of the external hydrophobes and the molecular weight of the HEURs influence the strength of the associative network. Longer external hydrophobes promote network formation (lower CAC) $)^{13,15}$ and strengthen the network (increase viscosity). ${ }^{13,18}$ 
The shape of the external hydrophobe also matters; HEURs with small, cyclic external hydrophobes cannot form micelles or form an associative network. ${ }^{16}$

As the molecular weight of HEURs increases, external hydrophobe concentration relative to that of lower molecular weight HEUR (holding the weight fraction of HEUR constant) decreases, ${ }^{10}$ which decreases the number of bridges (decreases low-shear Newtonian viscosity and increases CAC). ${ }^{11-13,15,18,26}$ Also, as an extension of the molecular weight effect, HEURs with higher polydispersity indexes (PDI) have higher lower viscosities than those with lower PDI. ${ }^{17}$ However, the viscosity can increase with increasing molecular weight until the number average molecular weight $\left(M_{n}\right)$ exceeded $18 \mathrm{kDa} .{ }^{10}$ Kaczmarski and Glass have attributed the increase in viscosity to the decreased tendency for external hydrophobes to associate intramolecularly, ${ }^{10}$ which promotes bridge formation (raises viscosity) due to increased distance between the external hydrophobes. ${ }^{10}$ However, to the author's knowledge, no follow up study has been conducted to investigate that observed relationship between molecular weight and viscosity.

\subsection{Rheology and Phase Behavior of Waterborne Latex / HEUR Mixtures}

When latex is added to a HEUR / water system, HEUR hydrophobes can associate with the hydrophobic portions of the latex particle surface ${ }^{21}$ (Figure 6). Such interactions are weak and are broken under shear, ${ }^{21}$ though the strength of those interactions is increased as external hydrophobe length is increased. ${ }^{27}$ When HEUR concentration is high, latex particles are dispersed throughout the system and the rheology shows Newtonian, shear thickening, and shear thinning behaviors. ${ }^{13-14,21,23}$ Yet, when HEUR concentration is low, 
latex particles can approach one another and flocculate. ${ }^{1}$ The bridging of latex particles by HEURs maintains the flocculated structures (bridging flocculation mechanism), though when shear is applied the flocculates are separated. ${ }^{1}$ Once shear is removed the flocculated structures are regenerated. ${ }^{1}$ The system then experiences syneresis as flocculated particles settle to the bottom of the system, generating a particle-poor top layer and a particle-rich bottom layer.

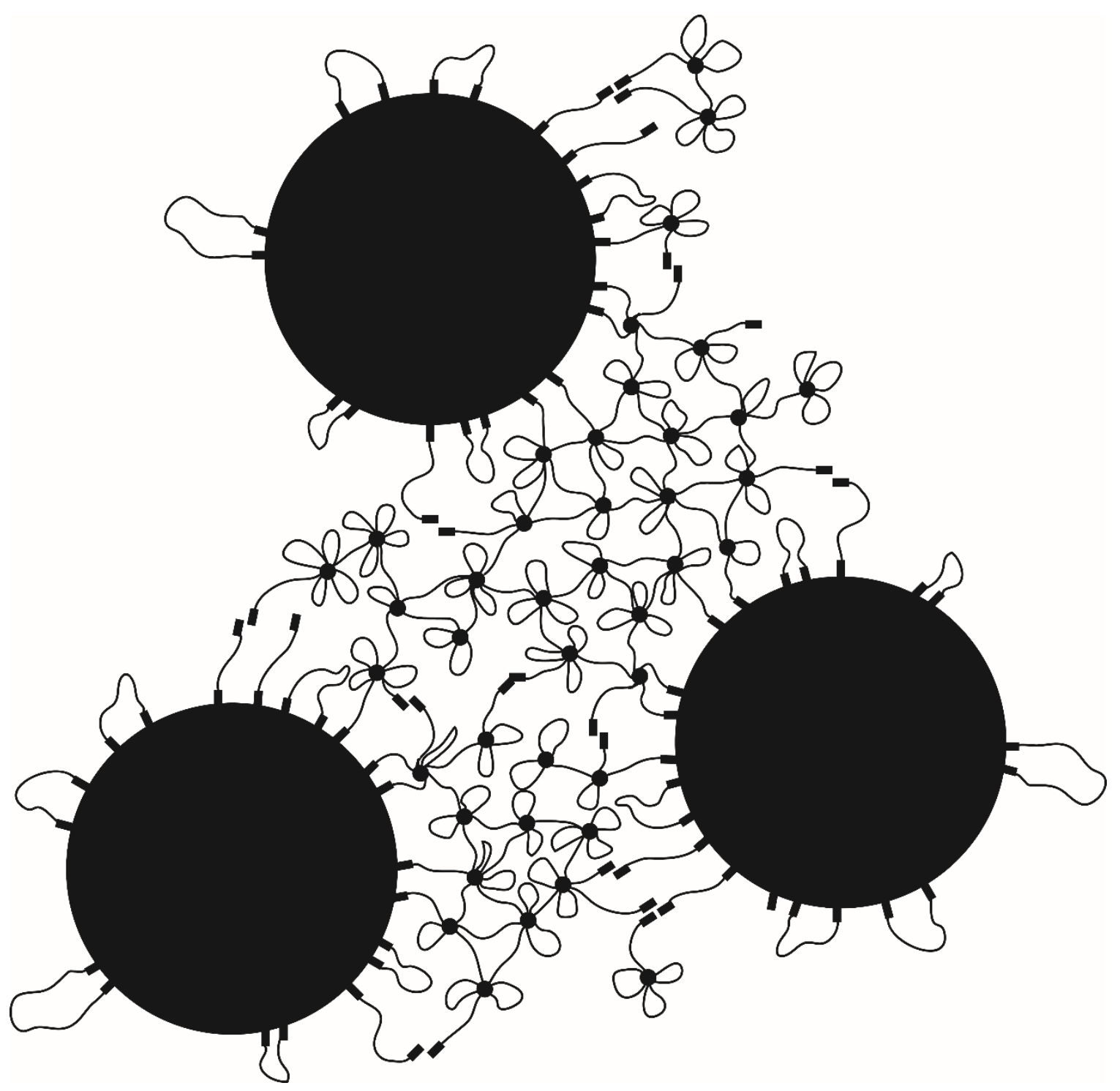

Figure 6. Schematic of Latex-HEUR Interactions. 


\subsection{Impact of Surfactants and Cosolvents}

When surfactant (anionic or nonionic) is added to aqueous HEUR solution or waterborne latex / HEUR mixtures, micelles that comprise surfactant and HEUR (mixed micelles) are formed. ${ }^{23,28}$ The addition of small quantities of surfactant increase the viscosity by increasing the number of micelles in the system and thus the fraction of HEURs that are in bridge conformation ${ }^{23}$ (Figure 7). However, above a critical surfactant concentration, the viscosity instead decreases with increasing surfactant concentration because the number of unbridged micelles exceeds the number of bridged micelles. ${ }^{23}$ The value of the viscosity maxima increases and occurs at lower critical micelle concentration when anionic surfactant is used instead of nonionic. ${ }^{28}$ This occurs as a result of electrostatic repulsion among the polar head groups of the surfactant, which decreases micelle size and generates more micelles per mole of surfactant. ${ }^{28}$

In addition to generating micelles, surfactant can adsorb to the latex particle surfaces and displace HEUR hydrophobes from the latex particle surfaces. ${ }^{14,27}$ This can alleviate bridging flocculation in waterborne latex / HEUR mixtures. ${ }^{1}$ However, when surfactant concentration is high and has displaced a majority of the HEUR polymers, unassociated HEUR can become excluded from the space between latex particles when the particles approach one another. ${ }^{1,4,29}$ This process generates osmotic pressure that generates latex particle flocculates by depletion flocculation. ${ }^{1,4,29}$ As with HEUR-based systems that experience bridging flocculation, flocculated particles settle and the system phase separates.

Cosolvents, such as butyl carbitol, can also decrease the adsorption of HEUR to latex particle surfaces, ${ }^{1,26,30}$ though such is accomplished by the decreased polarity of the 
continuous phase as opposed to direct competition for latex particle surface. ${ }^{26}$ As such, the desorption of HEUR from latex occurs more gradually. ${ }^{26,30}$ Furthermore, cosolvents disrupt hydrophobic associations between the external hydrophobes, which decreases viscosity as cosolvent concentration in the continuous phase increases. ${ }^{26,30}$ However, the viscosity does not decrease any further once the cosolvent concentration reaches $10-40$ $\mathrm{wt} \%$ of the continuous phase. ${ }^{26,30}$

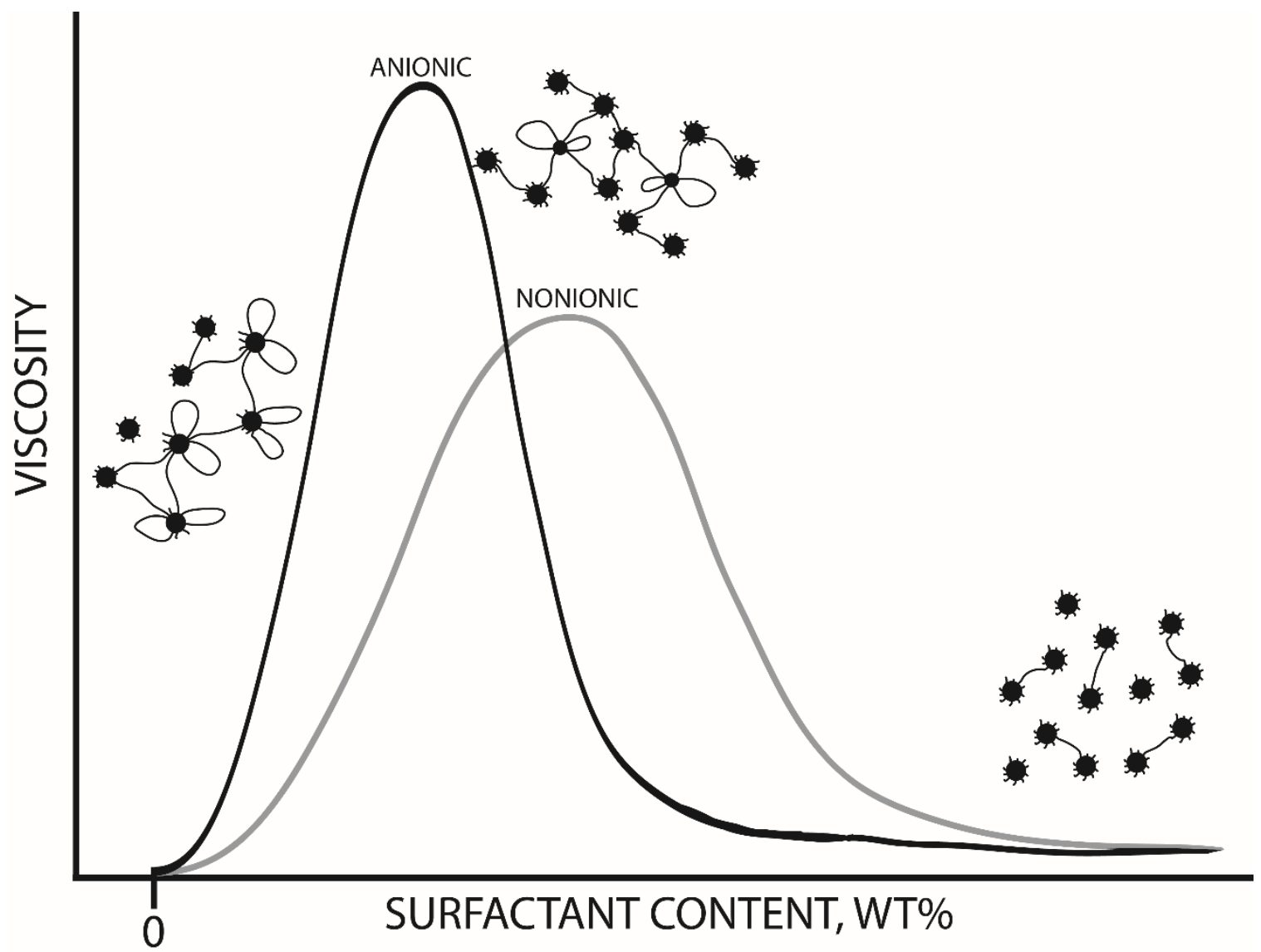

Figure 7. Effect of Surfactant Concentration on HEUR Solution Viscosity. 


\subsection{Dispersion Phase Diagrams}

The phase behaviors of HEUR-based systems can be summarized with dispersion phase diagrams (DPDs). DPDs are Cartesian plots that outline the phase behavior of AT systems as AT (y-axis) and surfactant (x-axis) concentration are varied (holding all else constant), ${ }^{1}$ as shown in Figure 8 . These diagrams originally appeared in Kostansek's study on the phase behavior of waterborne latex / HEUR / surfactant mixtures, ${ }^{1}$ though DPDs have also been used to study more complex systems, including fully formulated commercial paints. $^{4}$

Three regions are observed in the DPDs of waterborne latex / HEUR / surfactant mixtures: 1) a phase-separated region at low HEUR and surfactant concentration, 2) a stable dispersed region at medium HEUR and surfactant concentration, and 3) a phaseseparated region at high HEUR and surfactant concentration. ${ }^{1,4}$ Syneresis in the first and third regions occur by bridging and depletion flocculation, respectively. ${ }^{1}$ The sizes of the three regions are influenced by the composition of the system. For instance, the area of the depletion flocculation region expands to lower surfactant concentrations when anionic surfactant is used instead of nonionic surfactant. ${ }^{1}$ Also, the addition of cosolvent increases the size of the stable dispersion region and decreases the areas of both phaseseparated regions. ${ }^{1}$ 


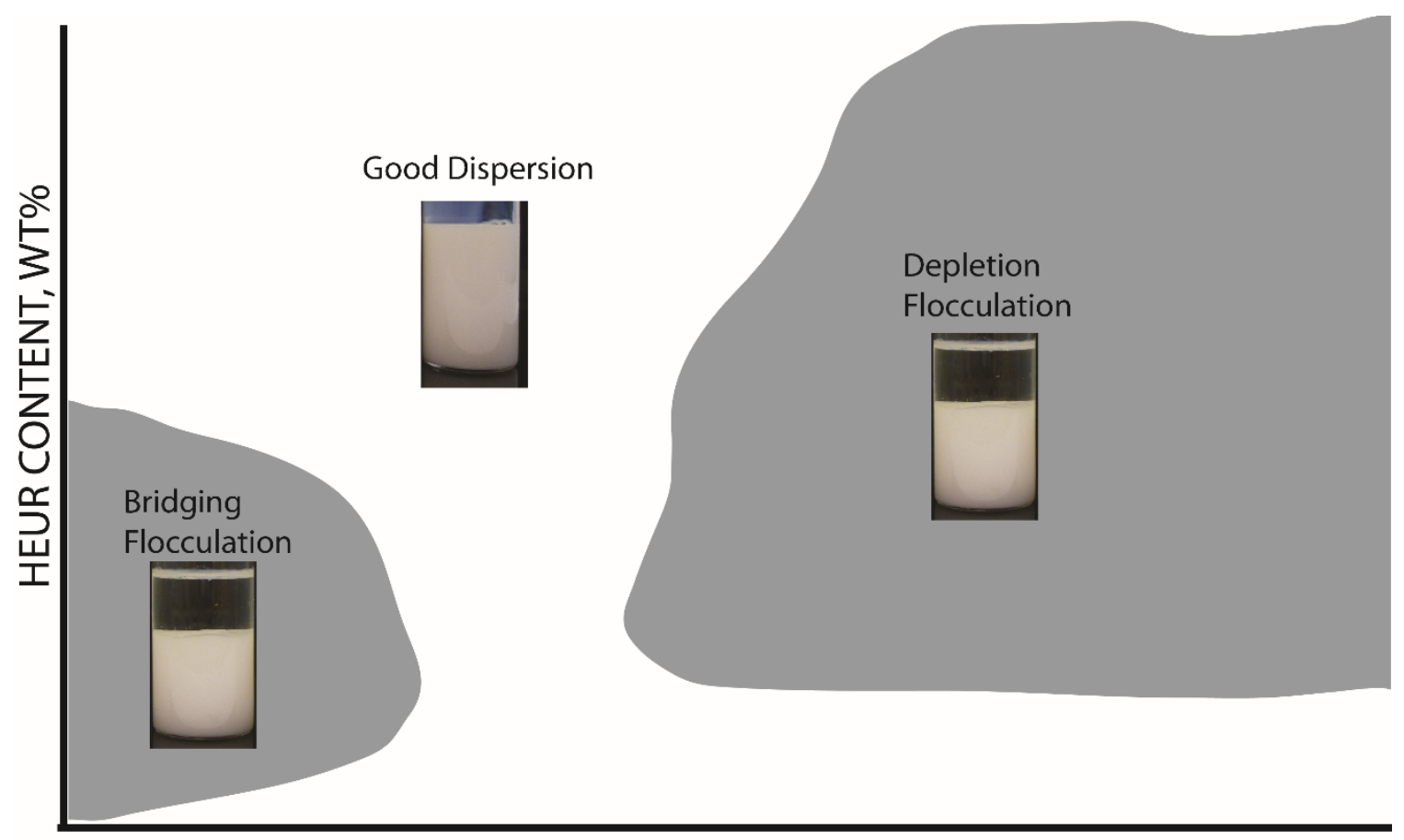

SURFACTANT CONTENT, WT\%

Figure 8. Dispersion Phase Diagrams of Latex / HEUR / Surfactant Mixtures.

\subsection{Modeling HEUR-based Systems with the Maxwell Model}

HEUR-based systems are special in that they can absorb stress like a solid as a consequence of the associative network and, flow like a liquid. Since they can show solid-like (elastic) behavior and liquid-like (viscous) behavior, HEUR-based systems are viscoelastic and additional information can be obtained by applying the single-mode Maxwell model. Other models that focus on the behaviors of viscoelastic fluids exist, but HEURs are often examined with the single-mode Maxwell model. ${ }^{25,31}$

In the single-mode Maxwell model, the system behaves like a dashpot and spring in series; the dashpot represents viscous behavior and the spring represents elastic behavior $^{31}$ (Figure 9a). When stress is applied or released, the spring immediately and reversibly deforms. ${ }^{31}$ The dashpot, on the other hand, is slow to respond and any resultant 
deformation is permanent. ${ }^{31}$ When stress is applied and held constant, the spring extends first $^{31}$ (Figure 9b) and then, as it returns to its unstretched state, the dashpot extends until the stress on the system decays to zero ${ }^{31}$ (Figure 9c). This phenomenon is called stress relaxation. $^{31}$

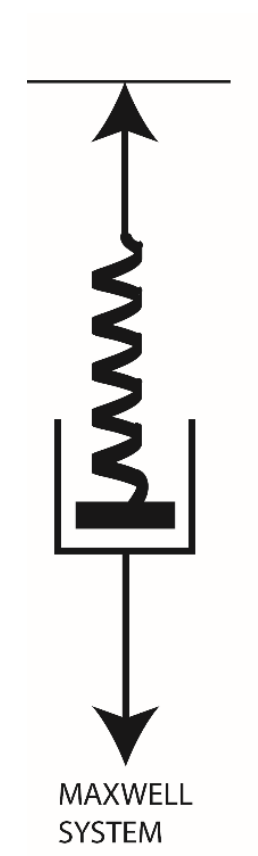

(a)

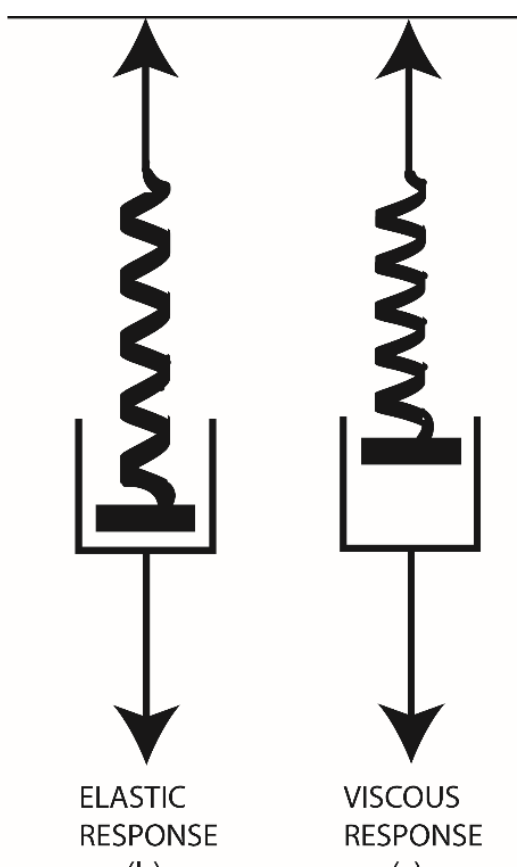

(c)

Figure 9. Maxwell Model at Rest (a) and Responding Elastically (b) or Viscously (c).

Three important parameters can be obtained using this model: relaxation time $(\tau)$, plateau modulus $\left(\mathrm{G}_{\infty}\right)$, and zero-shear viscosity $\left(\eta_{0}\right)$. The relaxation time is the time it takes for the network to release stress, which for aqueous HEUR solutions is dominated by the removal of hydrophobes from micelles. ${ }^{23,32}$ Similarly structured HEURs have similar relaxation times and mixtures of HEURs possess a number of relaxation times 
equal to the number of HEURs in the mixture and those relaxation times are equal to the relaxation times of the pure HEUR components that comprise the mixture. ${ }^{23}$

The plateau modulus is indicative of the strength of the associative network, ${ }^{25}$ and is related to the number of HEUR bridges in the system. ${ }^{13,23,32}$ The zero-shear viscosity is also indicative of network strength. Plots of zero-shear viscosity as a function of HEUR concentration have been used to compare the thickening efficiency of various HEURs and to estimate the value of the CAC. ${ }^{10-13,15,18}$ Such a plot is depicted in Figure 10. In such plots, the CAC is defined as the HEUR concentration beyond which the zero-shear viscosity increases rapidly. ${ }^{10-13,15,18}$

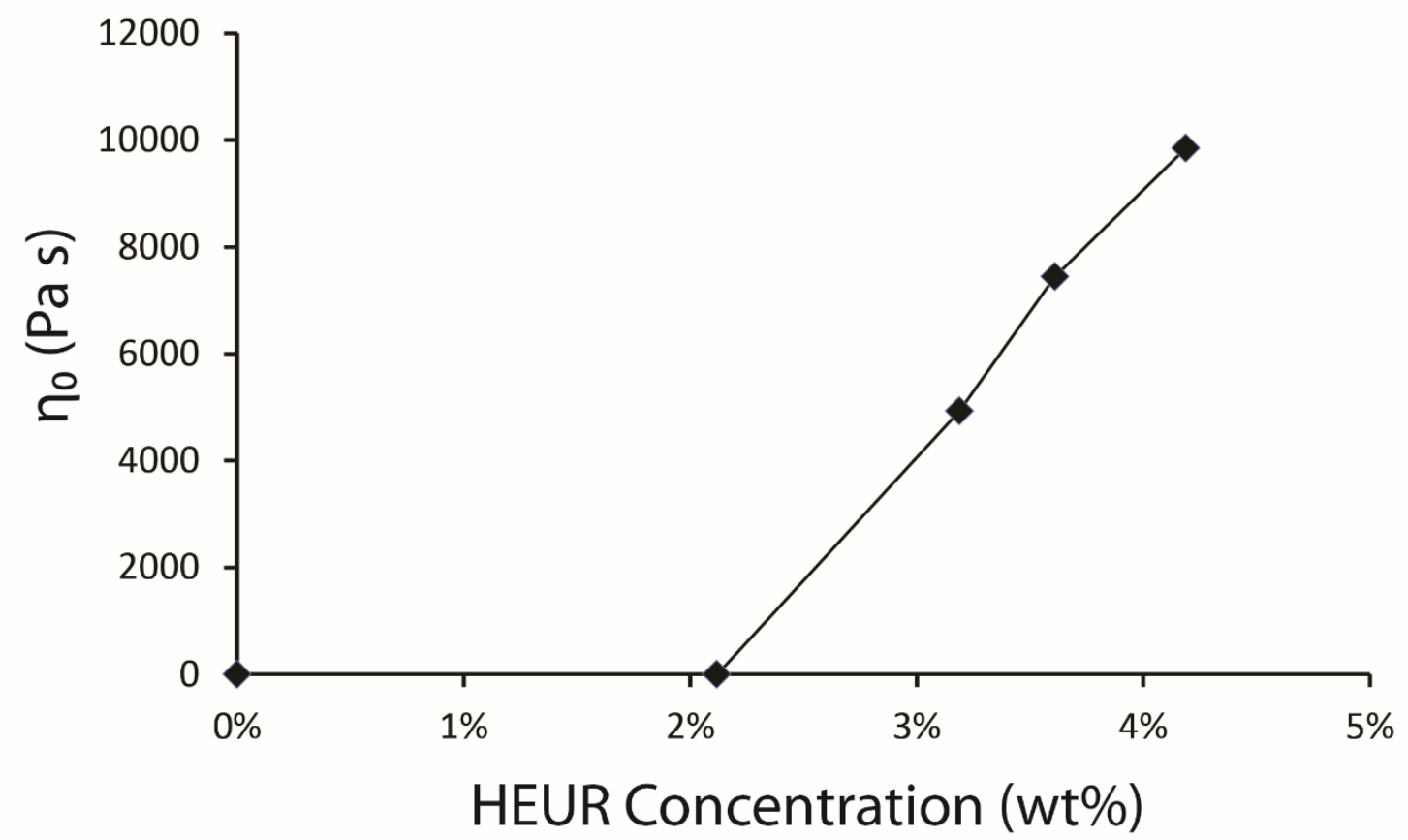

Figure 10. Zero-shear Viscosity of 2.1-4.2 wt\% Commercial Latex / Cal Poly HEUR (Samples 96-99) as a Function of HEUR Concentration. 
Those three parameters are obtained by subjecting the HEUR-based system to a sinusoidal stress and observing the sinusoidal response of the system. ${ }^{33}$ When both sinusoidal responses are in-phase, the system shows solid-like elastic behavior ${ }^{33}$ (Figure 11). If both responses are out-of-phase by $90^{\circ}$, the system instead shows liquid-like viscous behavior ${ }^{33}$ (Figure 11). When the curves are displaced between the two extremes $\left(0-90^{\circ}\right)$, the system shows a combination of elastic and viscous behavior. ${ }^{33}$ The displacement between both curves is called the phase angle $\delta$ and is related to the storage $\left(G^{\prime}\right)$ and loss $\left(G^{\prime \prime}\right)$ moduli by eq 2 .

$$
\frac{G^{\prime \prime}}{G^{\prime}}=\tan \delta
$$

To obtain the relaxation time, plateau modulus, and zero-shear viscosity, the system is first subject to an oscillatory strain and then an oscillatory frequency. In the first test the strain is varied at constant frequency (Figure 12), while in the second test the frequency is varied while the strain is held constant (Figure 13). Since both tests are dependent on one another, the strain sweep is run first to identify the linear viscoelastic region, the region where the storage and loss moduli are constant with varying strain (Figure 14). A strain within the linear viscoelastic region is chosen to be held constant during the frequency sweep. 


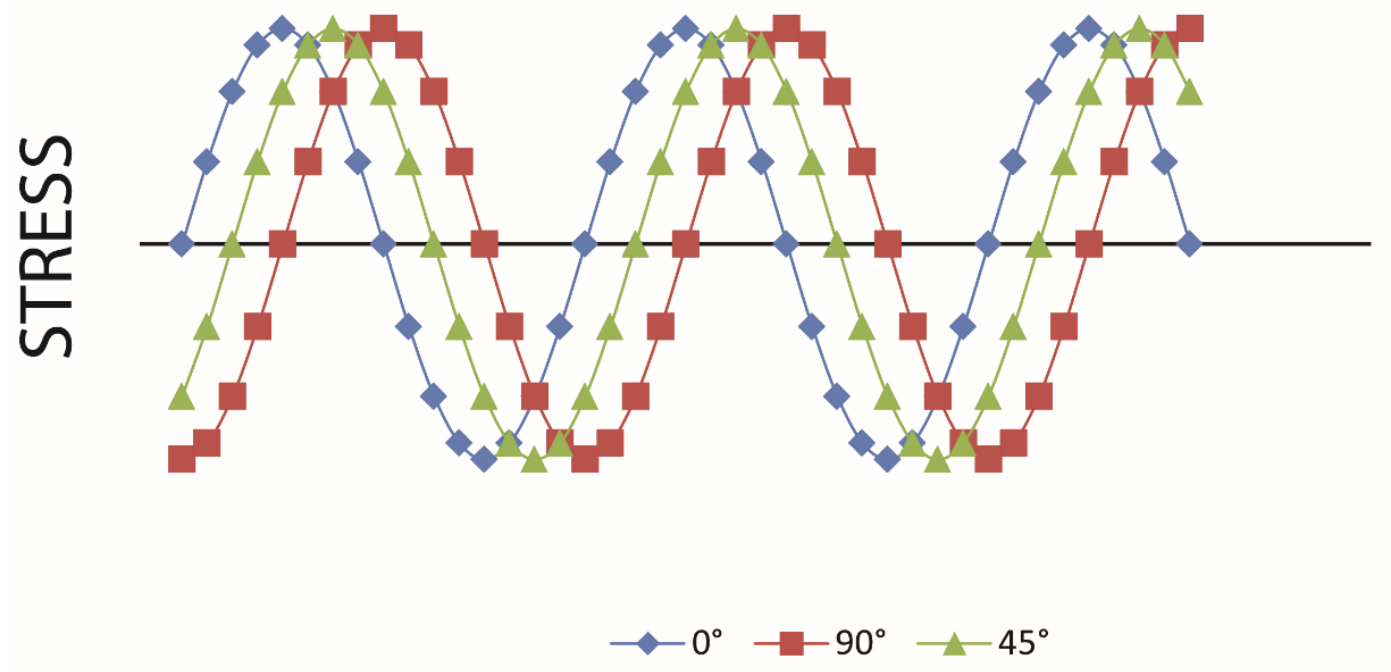

Figure 11. Sinusoidal Stress of System at 0, 90, and 45 Degree Phase Angles.

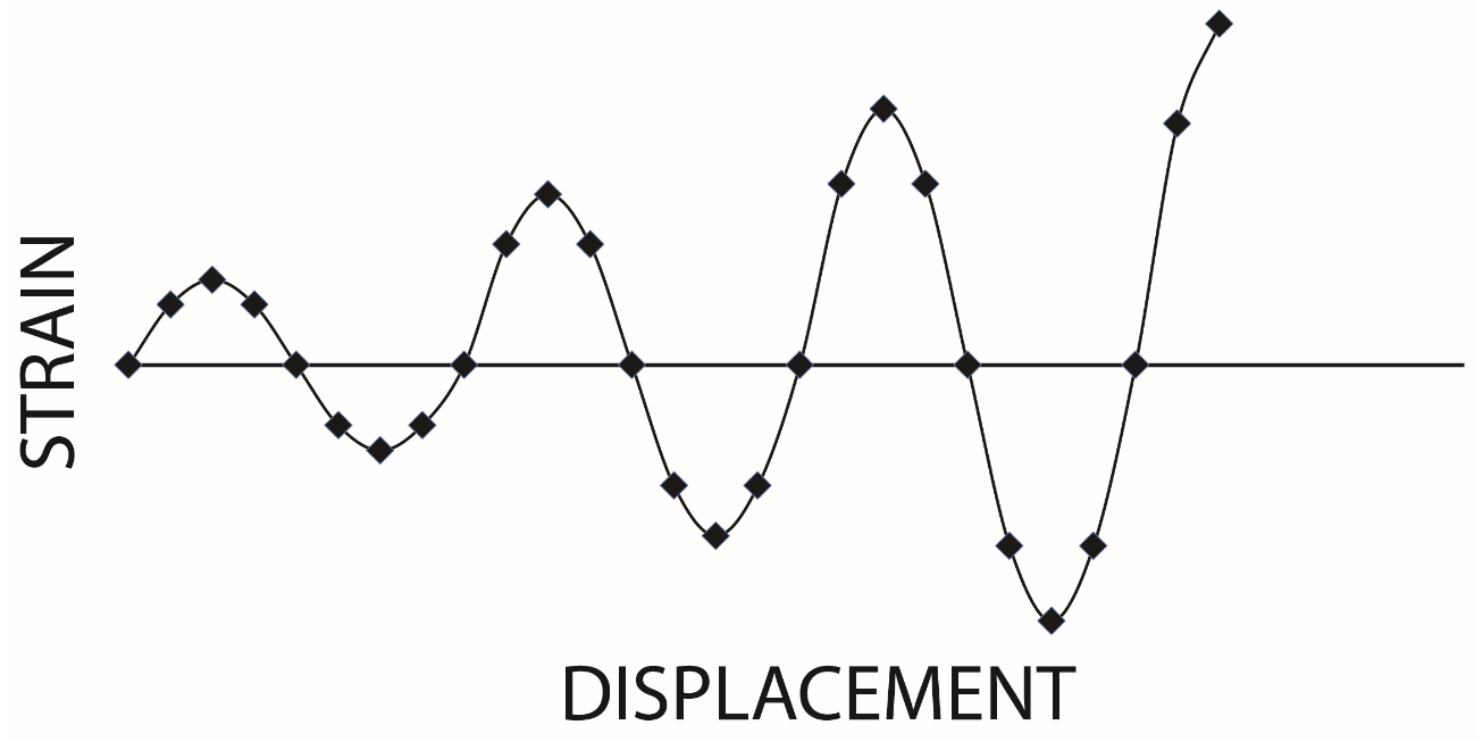

Figure 12. Strain Sweep, Whereby Strain Varied and Frequency is Held Constant. 


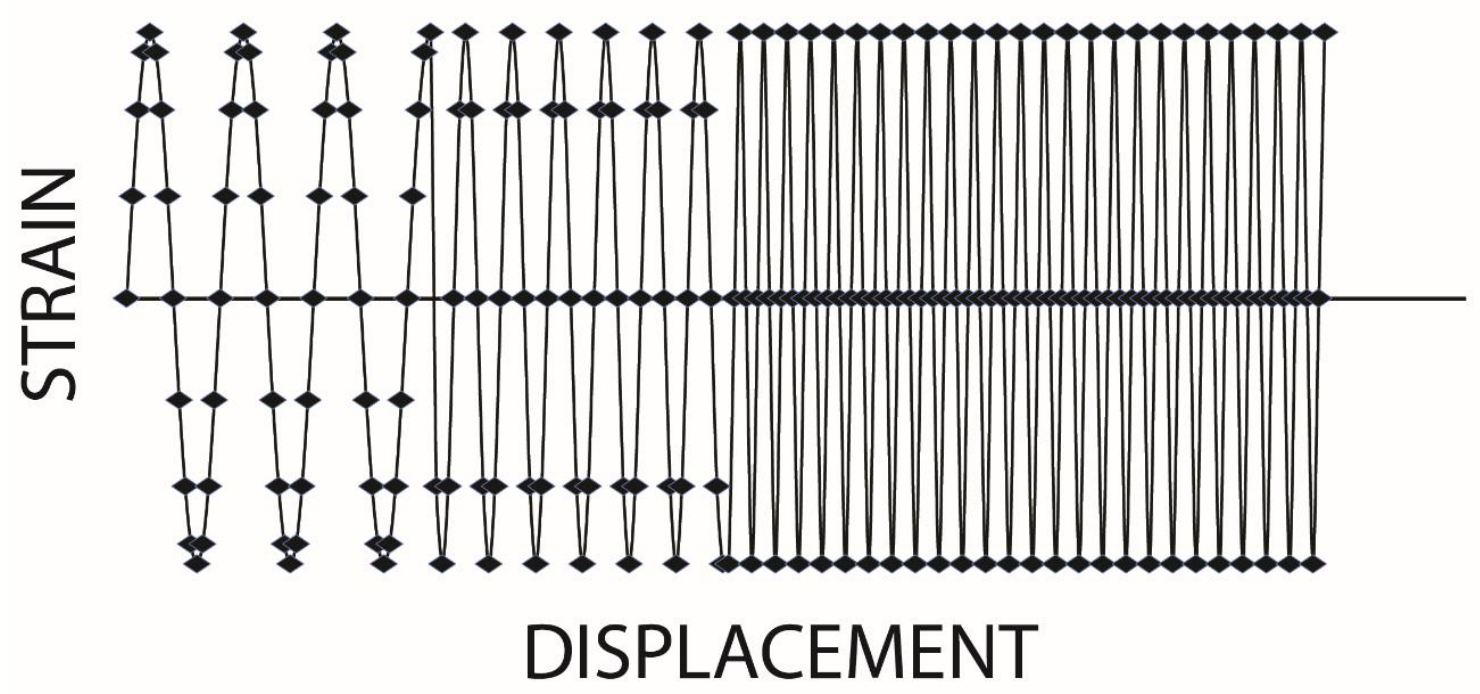

Figure 13. Frequency Sweep, Whereby Frequency Varied and Strain is Held Constant.

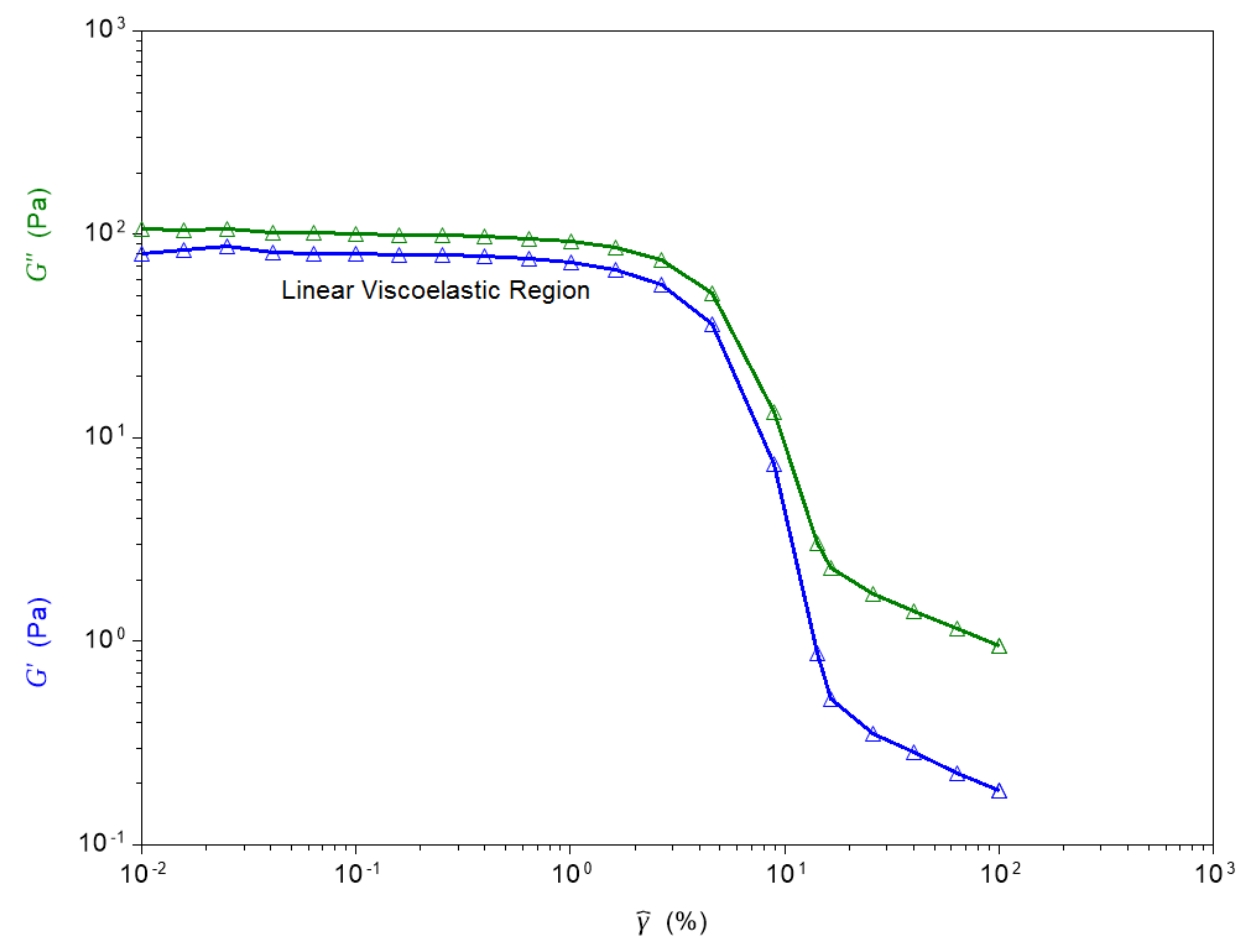

Figure 14. Strain Sweep (with Linear Viscoelastic Region) of $0.51 \mathrm{wt} \%$ Commercial Latex / Commercial HEUR Mixture. 
The relaxation time and plateau modulus are obtained from the frequency sweep by one of several means. For instance, plateau modulus can be extrapolated from a ColeCole plot (eq 3). ${ }^{23,25,34}$ Such a plot is shown in Figure 15.

$$
G^{\prime \prime}(\omega)=\left[G^{\prime}(\omega) G_{\infty}-G^{\prime}(\omega)^{2}\right]^{m}
$$

In a Cole-Cole plot, plateau modulus equals the value at which the semicircle intersects the positive $\mathrm{x}$-axis. ${ }^{25}$ Furthermore, if eq 3 completely overlaps the experimental data when $m=0.50$, the Maxwell model is considered a good mathematical fit for that system. $^{25}$

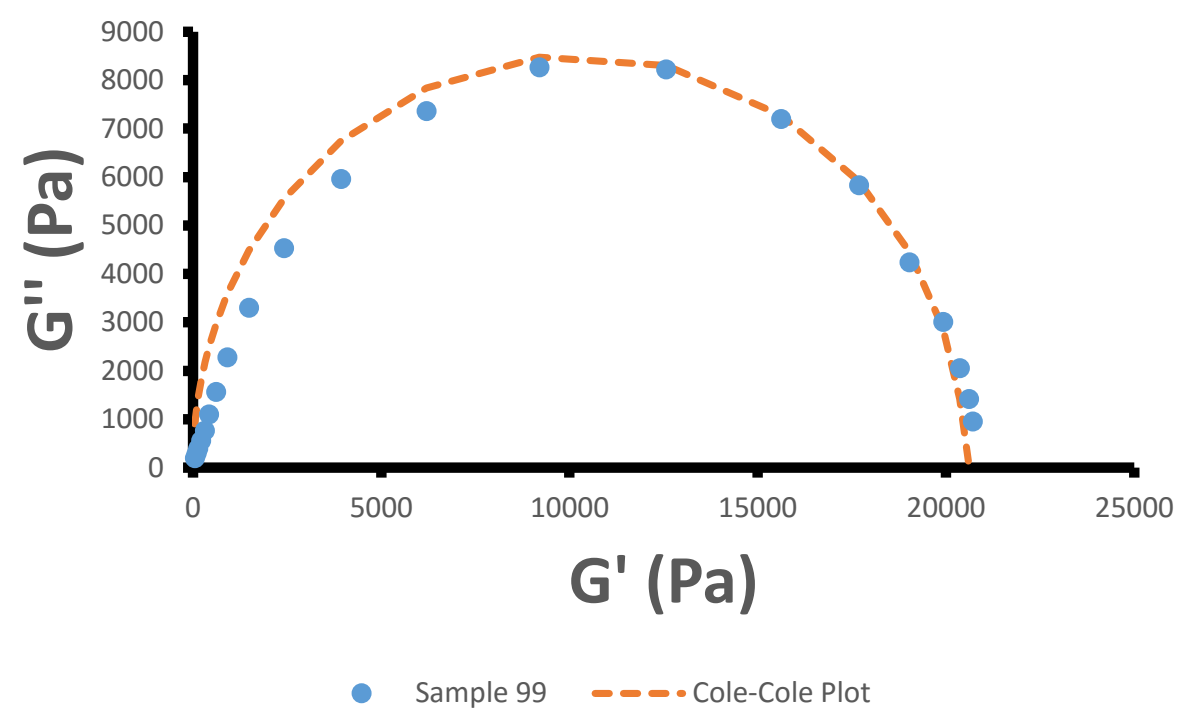

Figure 15. Cole-Cole Plot of 4.2 wt\% Commercial Latex / Commercial HEUR Mixture, Which Does Fit the Single-Mode Maxwell Model. 
Alternatively, plateau modulus can also be obtained by fitting eqs 4-5 to the storage and loss moduli (as functions of angular frequency $\omega(\mathrm{in} \mathrm{Hz})$ ) of the frequency sweep. ${ }^{13,25}$

$$
\begin{aligned}
& G^{\prime}(\omega)=\frac{G_{\infty} \omega^{2} \tau^{2}}{1+\omega^{2} \tau^{2}} \\
& G^{\prime \prime}(\omega)=\frac{G_{\infty} \omega \tau}{1+\omega^{2} \tau^{2}}
\end{aligned}
$$

The relaxation time can also be obtained from eqs 4-5, though it is more easily estimated from the reciprocal of the frequency where the storage and loss moduli intersect, and beyond which $G^{\prime}>G^{\prime \prime} .{ }^{35}$ The relaxation time can also be estimated from stress relaxation experiments. ${ }^{31}$ In stress relaxation experiments, the time $t$ for the value of the initial maintained stress $\gamma_{0}$ to fall to $\gamma_{0} / \mathrm{e}$ is the relaxation time (eq 6). ${ }^{31}$

$$
\mathrm{t}_{\gamma_{0} \rightarrow \frac{\gamma_{0}}{\mathrm{e}}}=\tau
$$

Lastly, the zero-shear viscosity is obtained from the product of the relaxation time and plateau modulus (eq 7), ${ }^{34}$ though in theory its value should be close to the low-shear (below $1 \mathrm{~s}^{-1}$ ) viscosity of the system of study.

$$
\eta_{0}=\mathrm{G}_{\infty} \tau
$$


2. Materials and Methods

\subsection{Materials}

Octadecyl isocyanate, dibutyltin dilaurate (T12), and PEG of $20 \mathrm{kDa}$ number average molecular weight (PEG20) were obtained from Sigma Aldrich. Anhydrous diethyl ether, acetone, and toluene were acquired from Fischer Scientific. Rhoplex AC261-LF (commercial latex) and Acrysol RM-825 (commercial HEUR) were provided by the Dow Chemical Company. Acrylic latex (DJ-I-043) was provided by Daisy Jauregui, a graduate student at Cal Poly. Toluene was dried with $4 \AA$ molecular sieves (acquired from EM Science) before use, and all other materials were used as received.

Several parameters of commercial HEUR, commercial latex, and DJ-I-043 are listed in Table 1. The molecular weight of commercial HEUR was determined by gel permeation chromatography (GPC) with a refractive index detector, THF eluent, and polystyrene standards. Particle sizes of DJ-I-043 and commercial HEUR were determined with a DynaPro NanoStar (a dynamic light scattering instrument which assumes spherical particles) using Dynamics V7 software (Wyatt Technology). A drop of latex was loaded into a disposable plastic cuvette (filled with nanopure water) from a disposable polycarbonate syringe that was outfitted with a syringe needle and $0.22 \mu \mathrm{m}$ syringe filter. Latex was mixed within the cuvette with the syringe needle. A $633 \mathrm{~nm}$ laser was employed and 10 acquisitions (5 seconds / acquisition) were averaged for each measurement. Particle sizes were obtained from the histogram window of the software. 
Table 1. Solids Content, Particle Diameter, and Density of Commercial Latex, ${ }^{36}$ Commercial HEUR, ${ }^{37}$ and Cal Poly Latex, and Molecular Weight and Polydispersity Index of Commercial HEUR. The Molecular Weight of Commercial HEUR Was Determined by Gel Permeation Chromatography.

Solids Content (wt\%) Particle Diameter (nm) Density $\left(\mathrm{g} \mathrm{mL}^{-1}\right)$ $M_{n}(\mathrm{kDa}) /$ PDI Rhoplex AC261-LF Acrysol RM-825 $^{\text {a }}$ DJ-I-043 $^{\text {b }}$

\begin{tabular}{|ccc|}
\hline 50 & 25 & 46.8 \\
140 & & 134 \\
1.06 & 1.04 & 1.06 \\
& $16,400(1.01)$ & \\
& $43,700(1.11)$ &
\end{tabular}

${ }^{\mathrm{a}}$ Continuous phase consists of $75 \mathrm{wt} \%$ water and $25 \mathrm{wt} \%$ butyl carbitol.

${ }^{\mathrm{b}}$ Contains at most $1.0 \mathrm{wt} \%$ sodium lauryl sulfate (determined from the initial masses of the reagents that were used to synthesize the latex) 


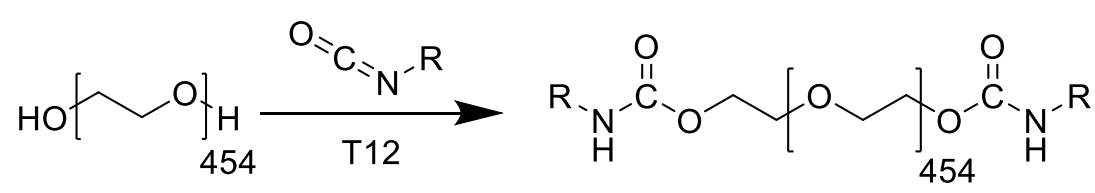

TS-I-036

Figure 16. Synthesis of Cal Poly HEUR from PEG20 and Octadecyl Isocyanate.
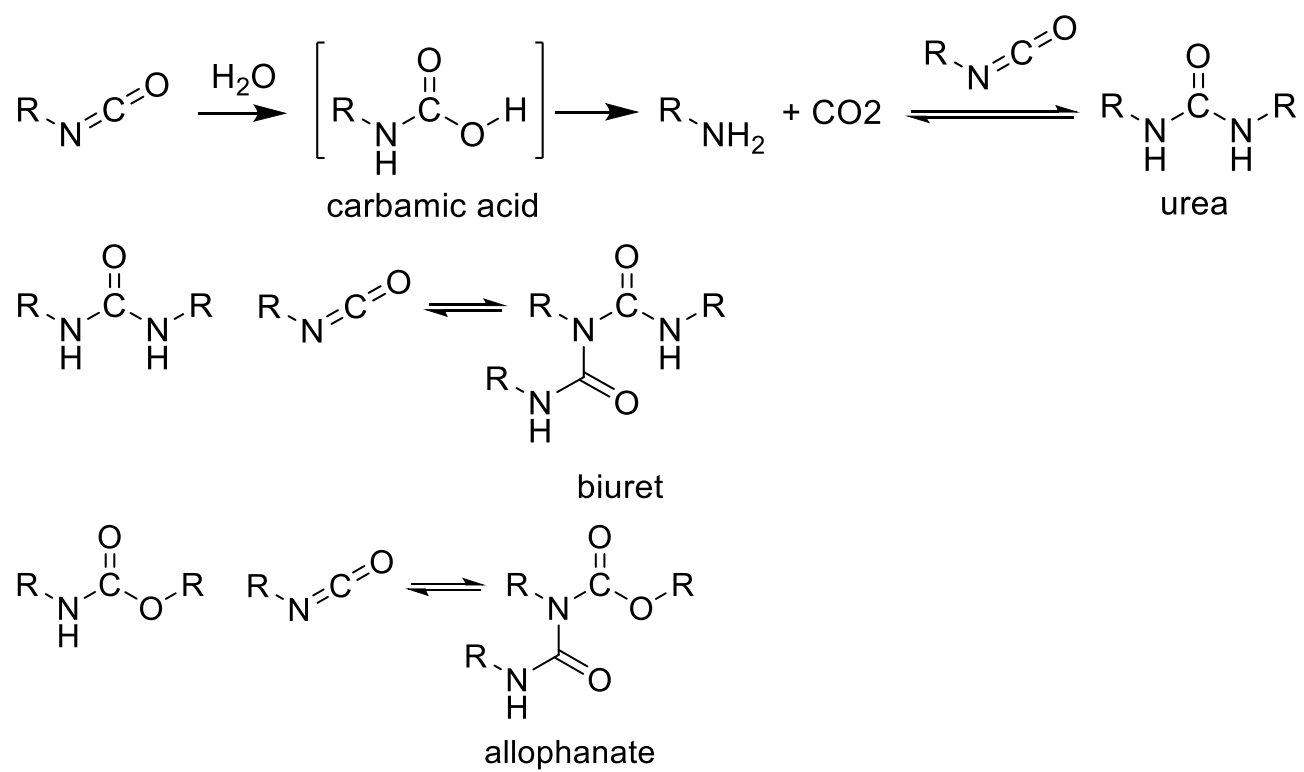

Figure 17. Synthesis of Urea from Isocyanate and Water (Top), Biuret from Urea Above $100{ }^{\circ} \mathrm{C}$ (Middle), and Allophanate from the Carbamate Group of HEUR Above $100{ }^{\circ} \mathrm{C}$ (Bottom).

In this study uni-HEUR (TS-I-036) was prepared from PEG20 and octadecyl isocyanate (Figure 16). PEG20 was dried beforehand to limit the formation of urea, and the reaction temperature was kept below $100{ }^{\circ} \mathrm{C}$ to limit biuret and allophanate production ${ }^{38}$ (Figure 17). Unlike other HEUR syntheses, which dry PEG via azeotropic distillation in 
toluene, ${ }^{9,10}$ PEG20 was dried by first melting and then drying under vacuum. The temperature was kept below $100{ }^{\circ} \mathrm{C}$ to limit the thermal degradation of PEG. ${ }^{18,39}$

A $100 \mathrm{~mL}$ 3-neck round bottom flask was outfitted with a short path distilling head, vacuum adaptor, and $100 \mathrm{~mL}$ round bottom flask (neck one); stirrer assembly (neck two); and universal inlet adaptor with hose connection (neck three). A capped glass rod containing a thermocouple was inserted through the inlet adaptor. The distilling head was capped with a rubber septum, and the nitrogen inlet of the short path distilling head was covered with a pipette bulb. The vacuum adaptor and universal inlet adaptor were both connected to a Schlenk line. The apparatus was heated with a hemispherical heating mantle.

Uni-HEUR was prepared from PEG20 and octadecyl isocyanate. PEG20 (26.4 g, $1.31 \mathrm{mmol}, 1.0$ equiv) was warmed to $76-86{ }^{\circ} \mathrm{C}$ and dried under vacuum for 3 hours under low agitation ( $25 \mathrm{rpm}$ ) until bubbles were removed from bulk. The vacuum trap was chilled with 1:1 ethanol : isopropanol. Afterwards, the glassware on neck one was then swapped for a rubber septum. A mixture of toluene $(15 \mathrm{~mL}), \mathrm{T} 12(52 \mu \mathrm{L}, 87 \mu \mathrm{mol}$, 0.066 equiv), and octadecyl isocyanate $(2.2 \mathrm{~mL}, 6.30 \mathrm{mmol}, 4.8$ equiv) that had been purged under nitrogen for 15 minutes was added to the reaction vessel with a purged disposable polycarbonate syringe. Reaction apparatus was heated to $80-85^{\circ} \mathrm{C}$ and reaction allowed to proceed under agitation $(25 \mathrm{rpm})$ for 2 hours under nitrogen atmosphere. 
After 2 hours, reaction was exposed to atmosphere and poured (while hot) into a 1 L flask. Reaction flask was rinsed with diethyl ether and white colored solids were transferred to the $1 \mathrm{~L}$ flask. Diethyl ether $(300 \mathrm{~mL})$ was added and solids were recovered by vacuum filtration (filtrate was clear colored). During drying, the $1 \mathrm{~L}$ flask was cleaned.

Solids were transferred to the cleaned flask, mixed with $300 \mathrm{~mL}$ acetone, and heated for several minutes on a warm hot plate (setting 6 out of 10) to $45^{\circ} \mathrm{C}$. Solids were recovered by vacuum filtration white the acetone was warm. Coarse filter paper was clogged very quickly, so solids were returned to the $1 \mathrm{~L}$ flask, mixed with $250 \mathrm{~mL}$ acetone, heated to $50{ }^{\circ} \mathrm{C}$, and then filtered by gravity filtration with new filter paper. Diethyl ether $(700 \mathrm{~mL})$ was added to the filtrate. Filtrate was stirred with a metal pole and white colored solids were recovered by vacuum filtration (resultant filtrate was clear colored). Solids were transferred to a glass jar and allowed to dry under ambient conditions.

Product was analyzed by Fourier-transform infrared spectroscopy (FTIR), and by proton nuclear magnetic resonance spectroscopy $\left({ }^{1} \mathrm{H}\right.$ NMR) in deuterated chloroform solvent. FTIR and ${ }^{1} \mathrm{H}$ NMR spectra of TS-I-036 and PEG20 are included in Appendices A-B, respectively. The molecular weight and PDI of TS-I-036 was determined by gel permeation chromatography (GPC) using Agilent 1200 Series GPC-SEC System that was outfitted with a refractive index detector with tetrahydrofuran eluent (at $1.0 \mathrm{~mL} / \mathrm{min}$ flow rate and $30^{\circ} \mathrm{C}$ ) and polystyrene standards. A GPC chromatograph of TS-I-036 is shown in Figure 19 and the molecular weight data is shown in Table 5. 


\subsection{Preparation and Analysis of Waterborne Latex / HEUR Mixtures}

In this section, materials are listed in the order to which they were added to a container before mixing. In this study, one set commercial latex / commercial HEUR (I), commercial latex / Cal Poly HEUR (II), and Cal Poly latex / Cal Poly HEUR (III) mixtures were prepared. First, aqueous HEUR solution was prepared by mixing HEUR powder and water in a Thinky mixer for two minutes. After one week, HEUR had completely dissolved in water. At that point, mixtures were prepared by mixing commercial latex, aqueous HEUR solution, and water in a AR-100 Thinky Mixer (centrifugal mixer) for two minutes (stock method), or by mixing latex, water, and HEUR powder with a metal spatula for 1-2 minutes (powder method). Mixtures that were prepared by the powder method were mixed again by hand 24 hours later for 3-5 minutes.

In all mixtures the volume fraction of latex was held constant and the concentration of HEUR was varied from 0-2 wt\% (Table 2), though more concentrated samples of II were prepared to see if the mixtures would form stable dispersions. Also, III above $1 \mathrm{wt} \%$ were not prepared due to limited quantity of latex. A similar amount of each mixtures was transferred to 11-dram vials, which were allowed to remain undisturbed for 7 days. The compositions and calculations for determining the compositions of all mixtures are provided in Appendix C. 
Table 2. General Compositions of Waterborne Latex / HEUR Mixtures.

\begin{tabular}{c|cc|cc|cc} 
Dispersion & HEUR & Wt\% & Latex & Vol\% & Preparation & Samples \\
\hline I & Acrysol & $0-2$ & Rhoplex & 25 & Stock & $81-95$ \\
\hline II & TS-I-036 & $0-4$ & Rhoplex & 25 & Stock & $62-76 ; 96-99$ \\
\hline II & TS-I-036 & $0-2$ & Rhoplex & 25 & Powder & $48-61$ \\
\hline III & TS-I-036 & $0-1$ & DJ-I-043 & 30 & Powder & $43-47$ \\
\hline
\end{tabular}

${ }^{a}$ Prepared by mixing water and HEUR powder for 1 minute in the Thinky Mixer,

followed by addition of commercial latex and subsequent mixing in the Thinky Mixer for 1 minute.

The percent syneresis (the ratio of the clear top-layer over the sample height) was measured on day 7 from photographs of the mixtures in a Byko-spectra light booth (under "Store" light) by using the plot profile tool of ImageJ. Details on the calculations are provided in Appendix D. Beyond day 7, syneresis heights were measured variably over two months.

After syneresis was checked on day 7, samples were mixed for 3 minutes with a metal spatula and an aliquot was removed for the rheology study (sample aliquots were discarded after testing). Viscosity profiles over a shear rate range of 0.01 to $1000 \mathrm{~s}^{-1}$ were obtained with a DHR-2 hybrid rheometer (TA instruments) equipped with a $40 \mathrm{~mm}, 2^{\circ}$ cone set at $55 \mu \mathrm{m}$ gap. The experimental parameters are listed in Table 3. Data points at low shear rates for mixtures with low HEUR concentrations may not be shown due to the sensitivity limit of the rheometer. Furthermore, due to time constraints, viscosity profiles were obtained on days 8-9 to determine to reproducibility of the viscosity profiles. 
Table 3. Parameters for the Rheological Testing of Waterborne Latex / HEUR Mixtures. Mixtures Were Subject to a Flow Peak Hold and then a Flow Peak Hold.

Samples 43-47 Step 1: Flow Peak Hold

\begin{tabular}{lcclcc} 
Parameter & Value & Units & Parameter & Value & Units \\
\hline Temperature & 25 & ${ }^{\circ} \mathrm{C}$ & Temperature & 25 & ${ }^{\circ} \mathrm{C}$ \\
Soak time & 0 & $\mathrm{~s}$ & Soak time & 0 & $\mathrm{~s}$ \\
Duration & 60 & $\mathrm{~s}$ & Duration & 60 & $\mathrm{~s}$ \\
Shear Rate & 100 & $\mathrm{~s}^{-1}$ & Shear Rate & 100 & $\mathrm{~s}^{-1}$ \\
\hline
\end{tabular}

\begin{tabular}{lcclcc}
\multicolumn{2}{c}{ Step 2: Flow Sweep } & & \multicolumn{2}{c}{ Step 2: Flow Sweep } \\
Parameter & Value & Units & Parameter & Value & Units \\
\hline Temperature & 25 & ${ }^{\circ} \mathrm{C}$ & Temperature & 25 & ${ }^{\circ} \mathrm{C}$ \\
Soak time & 0 & $\mathrm{~s}$ & Soak time & 0 & $\mathrm{~s}$ \\
Shear rate & $0.01-$ & $\mathrm{s}^{-1}$ & Shear rate & $0.01-1000$ & $\mathrm{~s}^{-1}$ \\
Points per decade & 1000 & & Points per decade & 5 & \\
Steady state sensing & 5 & & Steady state & Not & \\
Max. Equilibration & Checked & & sensing & Checked & \\
Time & 60 & $\mathrm{~s}$ & Equilibration time & 45 & $\mathrm{~s}$ \\
$\begin{array}{l}\text { Sample Period } \\
\text { \% Tolerance }\end{array}$ & 15 & $\mathrm{~s}$ & Sample period & 15 & $\mathrm{~s}$ \\
Consecutive within & 5 & & & &
\end{tabular}

For Samples 43-47 the "steady state sensing" option was selected for all runs; one data point was generated every 15 seconds. Ideally, the first five data points would be averaged and reported as a single value at the indicated shear rate provided all five values were within $5 \%$ of one another (consecutive within $5,5 \%$ tolerance). Since only four data points could be collected during the allotted time (60 second maximum equilibration time), the first four data points were averaged instead. To eliminate potential bias via the values collected at 15 and 30 seconds, the method of data collection was changed such that "steady state sensing" was not checked (Samples 48-99). In that case, sample was allowed to equilibrate at constant shear rate for 45 seconds and then the viscosity was 
measured during the next 15 seconds. The values generated during those 15 seconds were averaged by the rheometer and reported as a single value for that shear rate.

Strain and frequency sweeps of I-III mixtures were also obtained (Table 4) at variable times due to time constraints. A strain of $0.1 \%$ was within the linear viscoelastic region of most samples, and so strain was held at $0.1 \%$ during the frequency sweep.

Table 4. Parameters for the Dynamic Oscillatory Testing of Waterborne Latex / HEUR Mixtures. Mixtures Were Subject to a Strain Sweep and Then a Frequency Sweep.

\begin{tabular}{lcclcc}
\multicolumn{2}{c}{ Step 1: Strain Sweep } & & \multicolumn{2}{c}{ Step 2: Frequency Sweep } \\
Parameter & Value & Units & Parameter & Value & Units \\
\hline Temperature & 25 & ${ }^{\circ} \mathrm{C}$ & Temperature & 25 & ${ }^{\circ} \mathrm{C}$ \\
Soak time & 0 & $\mathrm{~s}$ & Soak time & 0 & $\mathrm{~s}$ \\
Wait for Temperature & Checked & & Wait for Temperature & Checked & \\
Frequency & 1.0 & $\mathrm{~Hz}$ & Strain & 0.1 & $\%$ \\
Strain Range & $0.01-100$ & $\%$ & Frequency Range & $0.01-100$ & $\mathrm{~Hz}$ \\
Points per Decade & 5 & & & &
\end{tabular}

Lastly, mixtures (S62-99) were analyzed with a Leica DM250CP modular polarization microscope at 5x magnification using Leica Application Suite v4.6 software to see if there was a clear transition between phase-separated and stable dispersions. Mixtures were stirred for 30 seconds before placement on a microscope slide and covered with a slide cover. Samples were not observed at 40x magnification because the images could not be focused. 


\section{Results and Discussion}

\subsection{HEUR Characterization}

The IR spectrum of TS-I-036 was indistinguishable from the spectra of PEG20. Also, the O-H stretch at $3000-3500 \mathrm{~cm}^{-1}$ was not visible in the spectra of PEG20. As such, reaction completion could not be verified by FTIR. The lack of difference between both spectra is likely due to the low fraction of urethane linkage and terminal alcohol in TS-I036 and PEG20.

In contrast, major differences between both materials were observed in the ${ }^{1} \mathrm{H}$ NMR spectra. In the spectra of PEG20, the series of peaks at 3.4-4.0 ppm corresponded to the methylene protons of the repeat unit. Similar peaks were observed in the spectrum of TS-I-036, as are peaks corresponding to the methylene groups of isocyanate ( 0.9 and $1.3 \mathrm{ppm})$ and those near the nitrogen of HEUR or urea (3.2 ppm). A set of multiplets at 4.2-4.4 ppm was observed in the spectrum of TS-I-036.

When PEG20 was modified with trifluoroacetic anhydride (TFAA) a triplet appeared at $4.5 \mathrm{ppm}$, which was attributed to the terminal methylene protons of unmodified PEG. No such peak was observed in the spectrum of TS-I-036 when it was also modified with TFAA. Regardless, the singlet peak at 1.6-1.8 ppm vanished from both spectra. Based on its placement, that peak was assumed to be water.

The extent of reaction and the composition of TS-I-036 was estimated from the ${ }^{1} \mathrm{H}$ NMR spectra of TFAA-treated HEUR (Table 5, Figure 18). Since the synthesis was carried out below $100^{\circ} \mathrm{C}$, the quantity of allophanate and biuret products in TS-I-036 was considered negligible. The extent of reaction (the mole fraction of PEG chain ends that 
had reacted with isocyanate) was obtained from eq 8 , where $H_{d}$ and $H_{f}$ are the integration per proton for $H_{d}$ and $H_{f}$ protons that are depicted in Figure 18B (eq 8).

$$
\text { extent of reaction }=\frac{H_{d}}{H_{d}+H_{f}}
$$

Since the chemical shifts of the methylene protons that are adjacent to the nitrogen protons in urea and HEUR are similar, the integration per proton of urea in the product $\left(H_{\text {urea }}\right)$ was estimated with eq 9.

$$
H_{\text {Urea }}=\frac{2 H_{c}-2 H_{d}}{2}=H_{c}-H_{d}
$$

With $H_{d}, H_{f}$, and $H_{\text {urea }}$, the mole fractions $n$ of HEUR, PEG20, and urea were calculated with eqs 10-12.

$$
\begin{gathered}
n_{\text {HEUR }}=\frac{H_{d}}{H_{d}+H_{f}+H_{\text {urea }}} \\
n_{P E G 20}=\frac{H_{f}}{H_{d}+H_{f}+H_{\text {urea }}} \\
n_{\text {urea }}=\frac{H_{\text {urea }}}{H_{d}+H_{f}+H_{\text {urea }}}
\end{gathered}
$$

From the calculated mole fractions of HEUR, PEG20, and urea, the weight fractions of those components were calculated with eqs $13-15$, where $n$ and $M$ are the mole fractions and molecular weights, respectively, of the designated component (Table $5)$. 


$$
\begin{aligned}
\text { Weight Fraction HEUR } & =\frac{n_{\text {HEUR }} M_{\text {HEUR }}}{n_{\text {HEUR }} M_{H E U R}+n_{P E G 20} M_{P E G 20}+n_{\text {urea }} M_{\text {urea }}} \\
\text { Weight Fraction PEG } & =\frac{n_{P E G 20} M_{P E G 20}}{n_{\text {HEUR }} M_{H E U R}+n_{P E G 20} M_{P E G 20}+n_{\text {urea }} M_{\text {urea }}} \\
\text { Weight Fraction Urea } & =\frac{n_{\text {urea }} M_{\text {urea }}}{n_{\text {HEUR }} M_{H E U R}+n_{P E G 20} M_{P E G 20}+n_{\text {urea }} M_{\text {urea }}}
\end{aligned}
$$

The molecular weight of TS-I-036 was obtained from the 8 minute peak of Figure 19 and those of commercial HEUR from the two peaks at 7-9 minutes. Peaks from 11-13 minutes in both chromatographs correspond to low molecular weight species, such as urea in TS-I-036. Unfortunately, the molecular weight of PEG20 could not be determined by GPC because PEG20 did not dissolve significantly in THF. Also, when an aliquot of that sample was injected into the GPC, no signal was observed over 15 minutes.

Kaczmarski and Glass have successfully obtained the molecular weights of PEG by GPC with THF eluent, though details regarding sample preparation were omitted. ${ }^{18}$ Barmar et al. ${ }^{13}$ have had success with chloroform eluent, though Cal Poly does not currently have the means to perform GPC with chloroform eluent.

Table 5. Molecular Weight, Polydispersity Index, and Composition of Cal Poly HEUR. The Molecular Weight and PDI Were Obtained by GPC and the Composition and Extent of Reaction by ${ }^{1} \mathrm{H}$ NMR.

\begin{tabular}{c|cc|ccc|c} 
& $\boldsymbol{M}_{\boldsymbol{n}}$ & PDI & \multicolumn{4}{|c|}{ Composition } \\
& $(\mathbf{k D a})$ & & HEUR & PEG & Urea & Extent of Reaction \\
\cline { 2 - 7 } TS-I-036 & 34,300 & 1.04 & 0.96 & 0 & 0.04 & 1.00
\end{tabular}


(A)
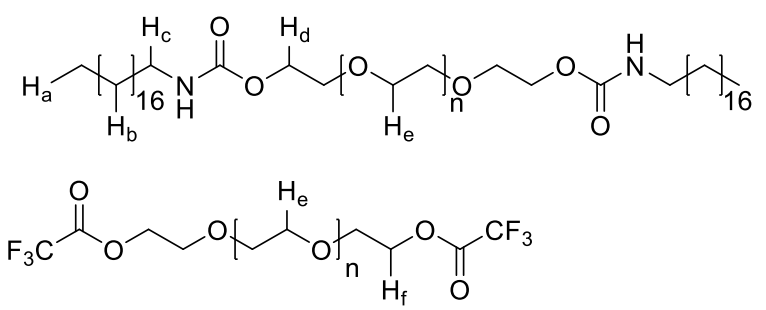

(B)

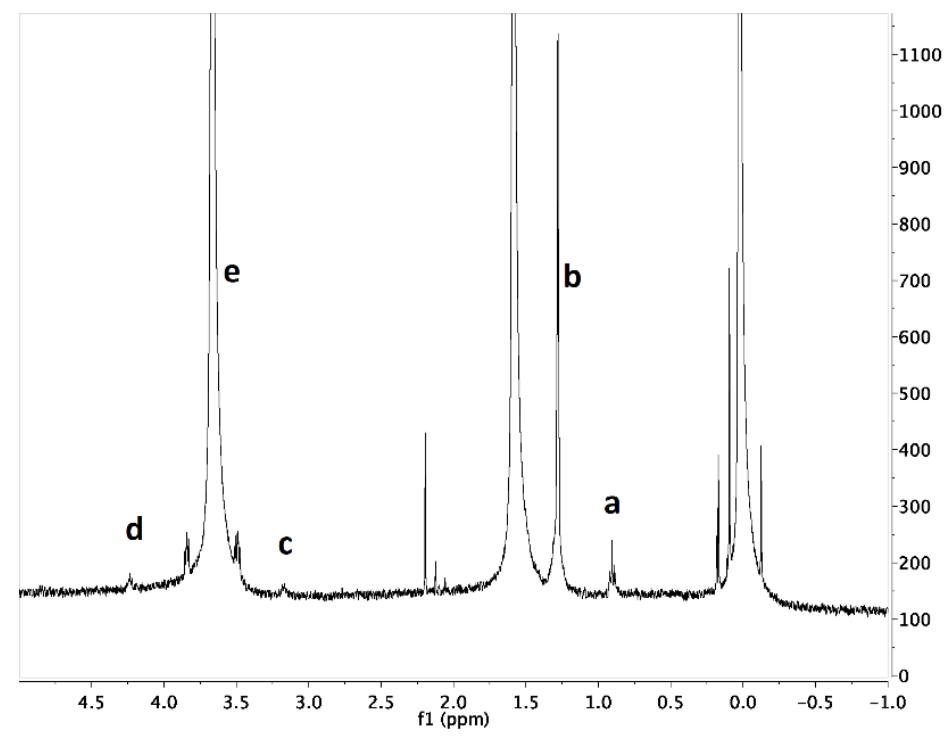

(C)

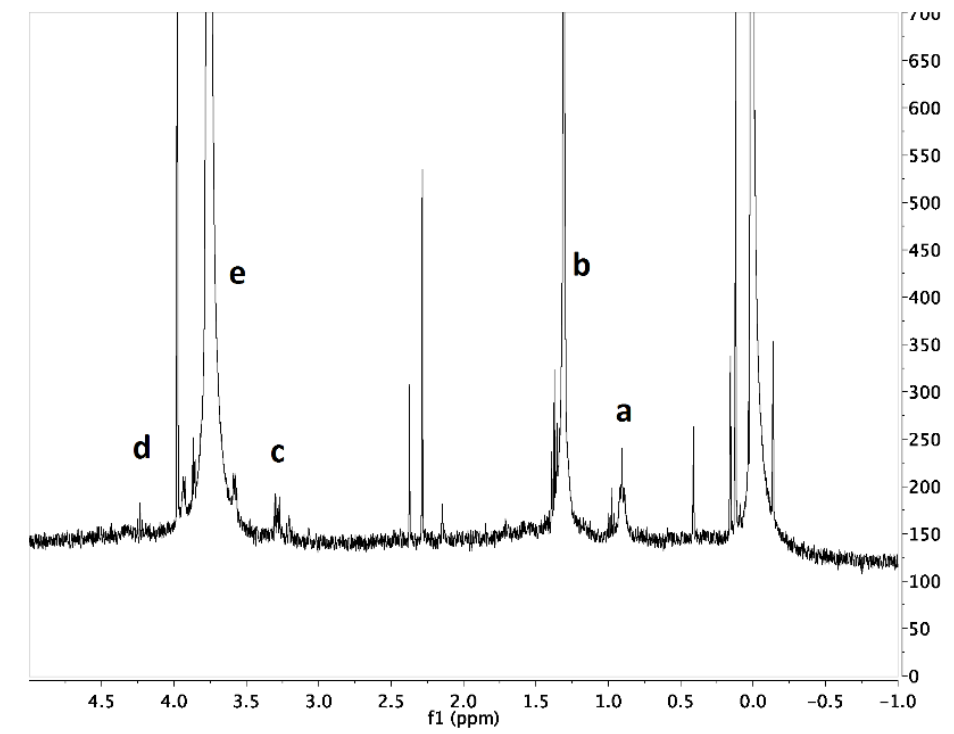

Figure 18. (A) Chemical Structures of uni-HEUR (Top) and TFAA End-capped PEG20 (Bottom); (B) ${ }^{1} \mathrm{H}$ NMR of TS-I-036; (C) ${ }^{1} \mathrm{H}$ NMR of TS-I-036 That Was Treated With TFAA. 


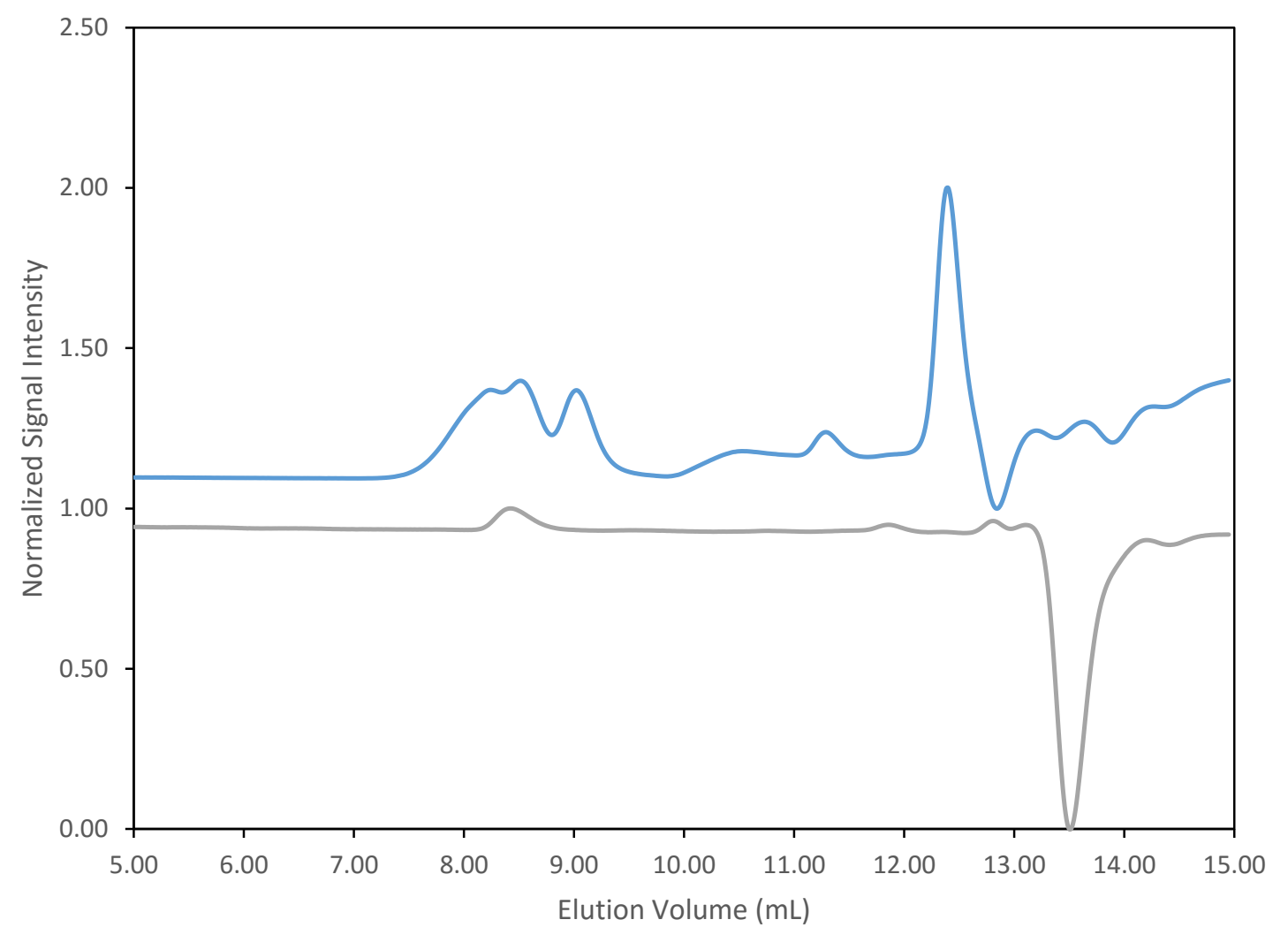

Figure 19. GPC Chromatographs of Commercial HEUR (Top) and Cal Poly HEUR (Bottom).

\subsection{Comparison of Powder and Stock Sample Preparation Methods}

Mixtures were initially prepared by the powder method, though such mixtures displayed a very viscous and opaque top layer (Figure 20). In mixtures that were prepared by the stock method the top layer was clear and not viscous (Figure 21), which indicated that HEUR was not well-dispersed in the mixtures that were prepared by the powder method. Additional evidence in support of this conclusion is the presence of large solid aggregates in mixtures that were prepared by the powder method. No such aggregates were observed in mixtures that were prepared by the stock method. Interestingly, the viscous top layer 
was not observed in powder method mixtures beyond day 7 and was replaced with a clear top layer.

As such, the stock method was used to better disperse HEUR in the mixtures. In the subsequent analysis, I and II were prepared by the stock method and III by the powder method. Since the powder method mixtures showed a viscous, opaque top layer on day 7, the percent syneresis of III on day 7 were assigned a value of zero.

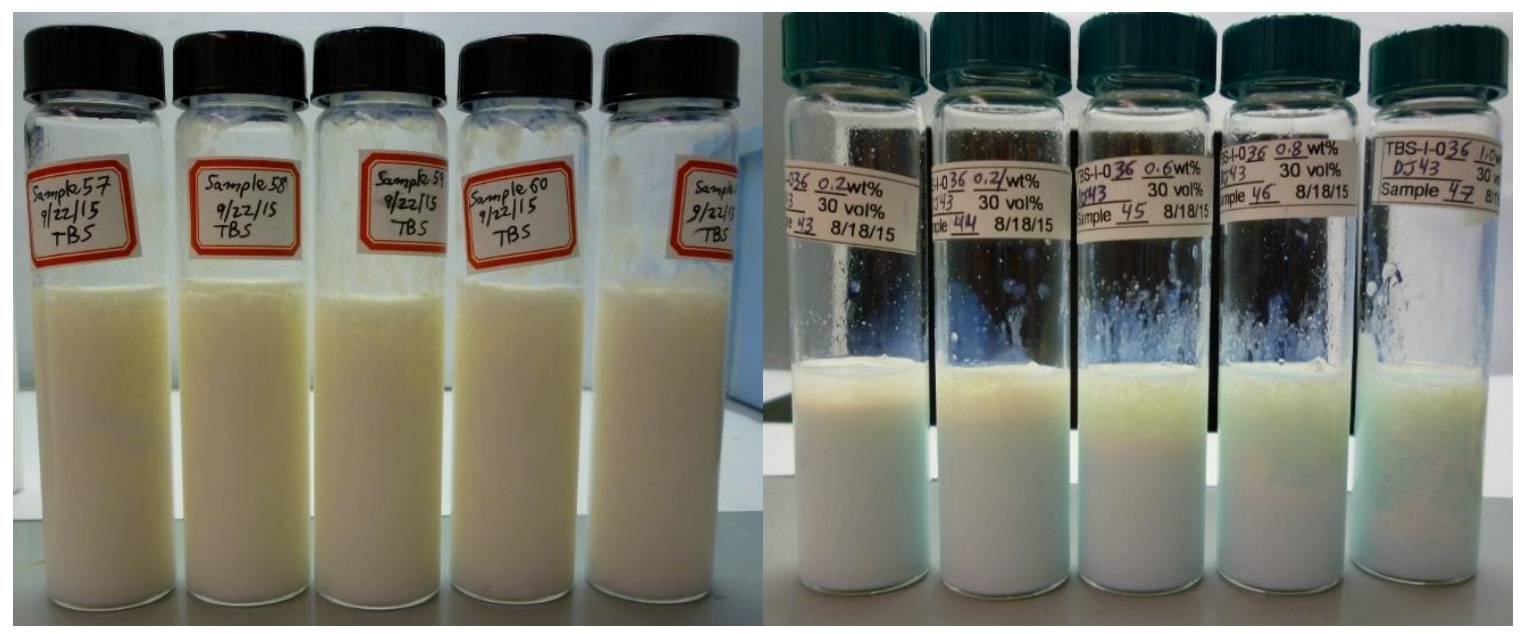

Figure 20. Viscous Top Layer in Commercial Latex / Cal Poly HEUR (Left), and Cal Poly Latex / Cal Poly HEUR Mixtures (Right) on Day 7. 


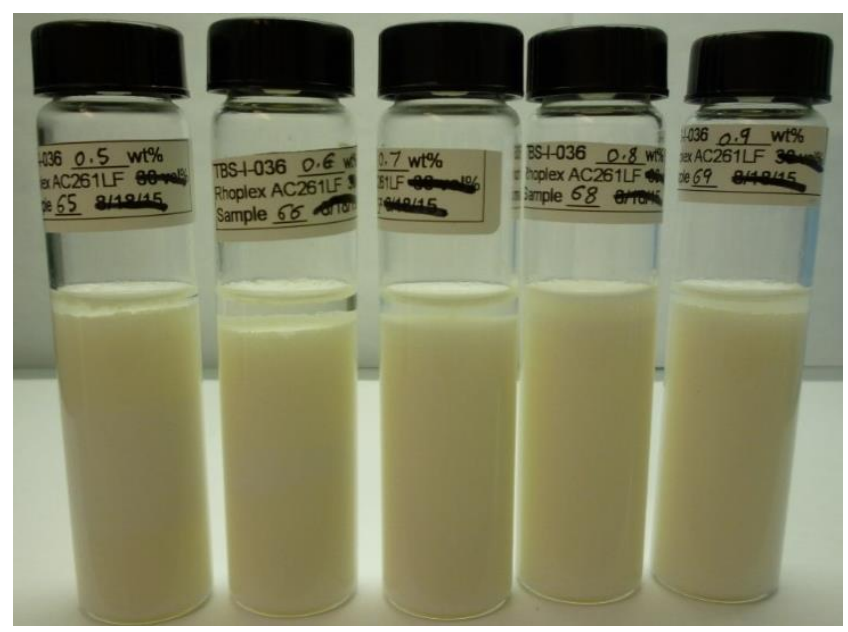

Figure 21. Clear Top Layer in Commercial Latex / Cal Poly HEUR Mixtures on Day 7.

\subsection{Phase Separation and Rheology}

Syneresis was observed at low HEUR concentrations in I and II, and was absent in the more concentrated mixtures (Table 6). Based on the DPDs of simple HEUR-based systems (Figure 8), the observed syneresis in I and II could be caused by bridging flocculation. However, the possibility that the observed syneresis could be caused by depletion flocculation cannot be discounted since the surfactant content of the commercial latex is unknown. Yet, the flocculation mechanism could be inferred by constructing a DPD from a series of $\mathbf{I}$ to which surfactant is added. Depending on the shape of the phase-separated region(s) of that DPD, the flocculation mechanism could be deduced. 
Table 6. Commercial Latex / Commercial HEUR (I), Commercial Latex / Cal Poly

HEUR (II), and Cal Poly Latex / Cal Poly HEUR Mixtures (III) That Showed Syneresis. The Concentrations of Mixtures that Showed Syneresis Are Listed in The Table.

\begin{tabular}{cc} 
System & HEUR $(\mathbf{w t} \%)$ \\
\hline I & $0.2-1.0$ \\
II & $0.3-2.0$ \\
III & None
\end{tabular}

Interestingly, syneresis was observed at a wide range of HEUR concentrations in II as opposed to I. This indicates significant compositional and structural differences between the commercial and Cal Poly HEUR, though further elucidation is limited without additional details about the commercial HEUR.

Due to time constraints, only one series of mixtures per latex / HEUR system was studied. Also, aliquots of the mixtures were removed to obtain viscosity profiles and were discarded after the test. The removal of aliquots reduces sample height, which can introduce significant deviations in percent syneresis via height dependent syneresis phenomena. Ideally, the duplicates of the mixtures would be prepared and aliquots would not be removed from the mixture.

However, since sample heights decreased, I and II were allowed to remain undisturbed for two weeks after rheological testing on days 7-9 (Figures 22-23). The percent values of those mixtures after 1-2 weeks at rest were generally within several percent of one another. When II were mixed again and allowed to remain undisturbed for 3 weeks, again the percent syneresis did not very by more than a few percent after 9 and 21 days at rest. This trend suggests that the percent syneresis of these mixtures is height- 
independent, though such could be confirmed by preparing identical samples with different sample heights.

A correlation is apparent when the syneresis trends and viscosity profiles are compared. The viscosity profiles of phase-separated I and II (Figures 24-25) show complex rheology and shear thickening between $1-100 \mathrm{~s}^{-1}$. When syneresis was absent in the I mixtures, the viscosity profile was Newtonian at low shear rates and shear thinning at high shear rates. This observation agrees with the syneresis-rheology correlation that was previously observed at Cal Poly. However, the viscosity profiles of dispersed (not phase-separated) II were not obtained because sample was ejected from the cone / plate geometry at shear rates near the onset of shear thickening $\left(\sim 1-100 \mathrm{~s}^{-1}\right)$ when the concentration of HEUR was greater than $0.4 \mathrm{wt} \%$ (Figure 26). Thus the high-shear rate viscosities in Figure 25 are of the remaining material beneath the cone / plate geometry. In addition, as the concentration of HEUR increased more material was ejected. Above $1.1 \mathrm{wt} \%$ HEUR a significant quantity of sample were ejected during the pre-shear step. As such, the viscosity profiles of $1.2-4.2 \mathrm{wt} \%$ HEUR were not obtained. Due to time constraints, the viscosity profiles in the absence of the pre-shear step were not obtained.

Thus, while the observed syneresis-rheology trend of phase-separated II agrees with that of the phase-separated I mixtures, a similar agreement for the dispersed mixtures is required to confirm that the correlation entirely applies to II.

In III a viscous top layer was observed on day 7 and syneresis was not observed, even after three months. The lack of syneresis might be due to the surfactant concentration in DJ-I-043 ( 1 wt\% sodium dodecyl sulfate), though such cannot be confirmed without the DPD of this system. 
Similar to the viscosity profiles of dispersed I, the viscosity profiles of III (Figure 27) showed Newtonian behavior at low shear rates and shear thinning behavior at high shear rates. This trend agrees with the syneresis-rheology correlation of dispersed I, though, as with phase-separated II, similar agreement for phase-separated III is required to confirm that the correlation of I applies entirely to III.

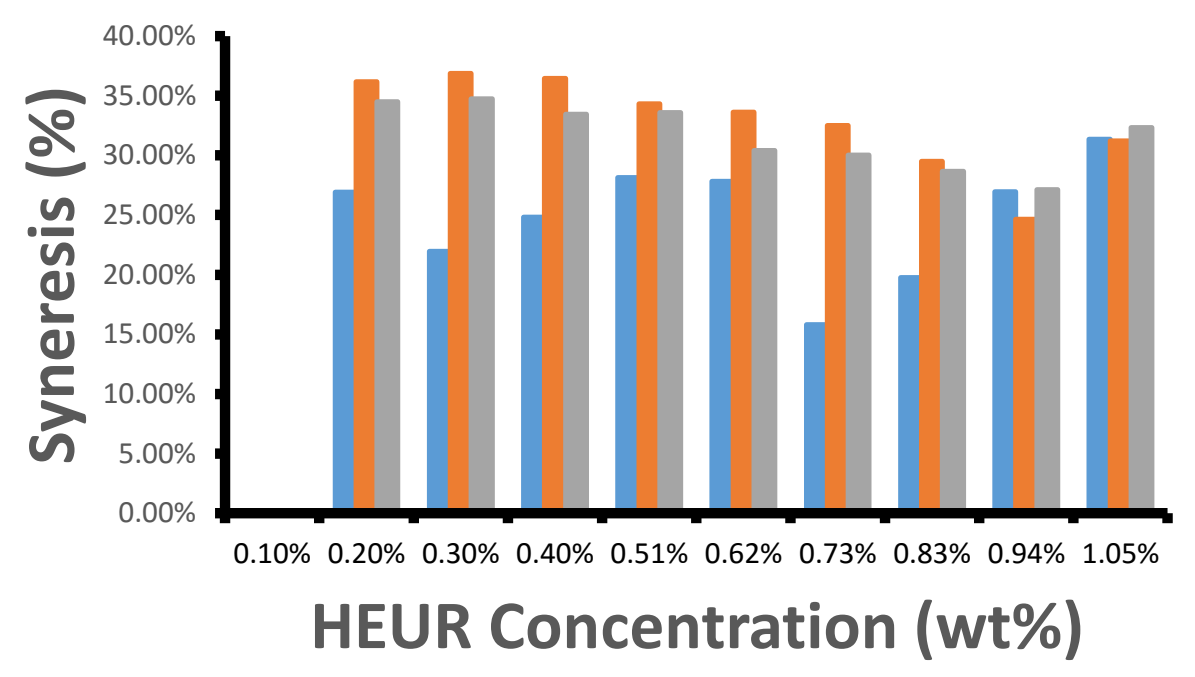

Day 7 Days Since Mixed 21 Days Since Mixed

Figure 22. Percent Syneresis in Commercial Latex / Commercial HEUR Mixtures on Day 7. After Rheological Testing Had Ended, Samples Were Allowed to Remain Undisturbed and Percent Syneresis Was Measured After 9 and 21 Days at Rest. 


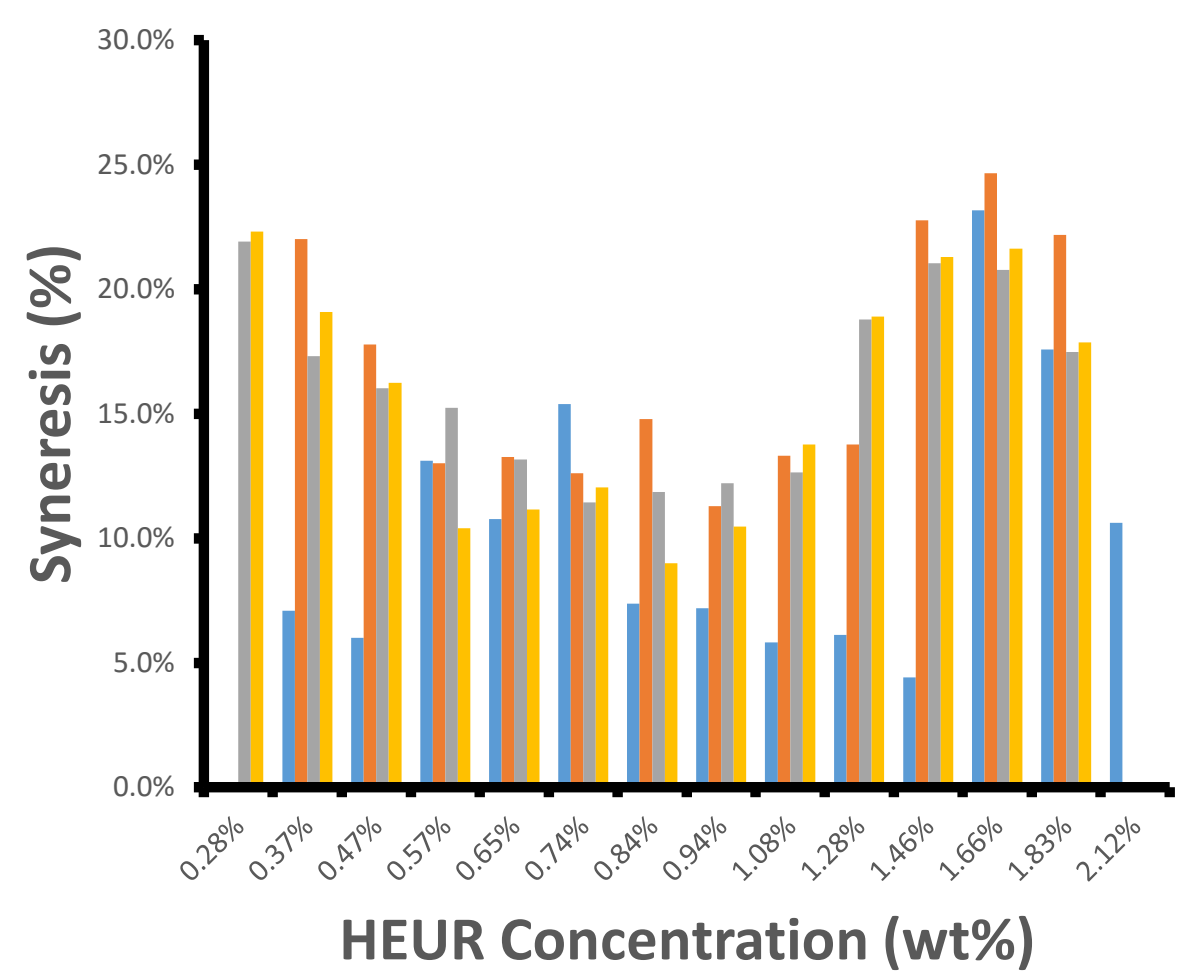

Day $7 \quad 7$ Days Since Mixed $\square 14$ Days Since Mixed $\square 21$ Days Sinced Mixed \#2

Figure 23. Percent Syneresis in Commercial Latex / Cal Poly HEUR Mixtures on Day 7. After Rheological Testing Had Ended, Samples Were Allowed to Remain Undisturbed and Percent Syneresis Was Measured After 7 and 14 Days at Rest. Samples Were Subject to a Second Round of Rheological Testing, Were Allowed to Remain Undisturbed for 21 Days, and Percent Syneresis Was Measured on that $21^{\text {st }}$ Day. 


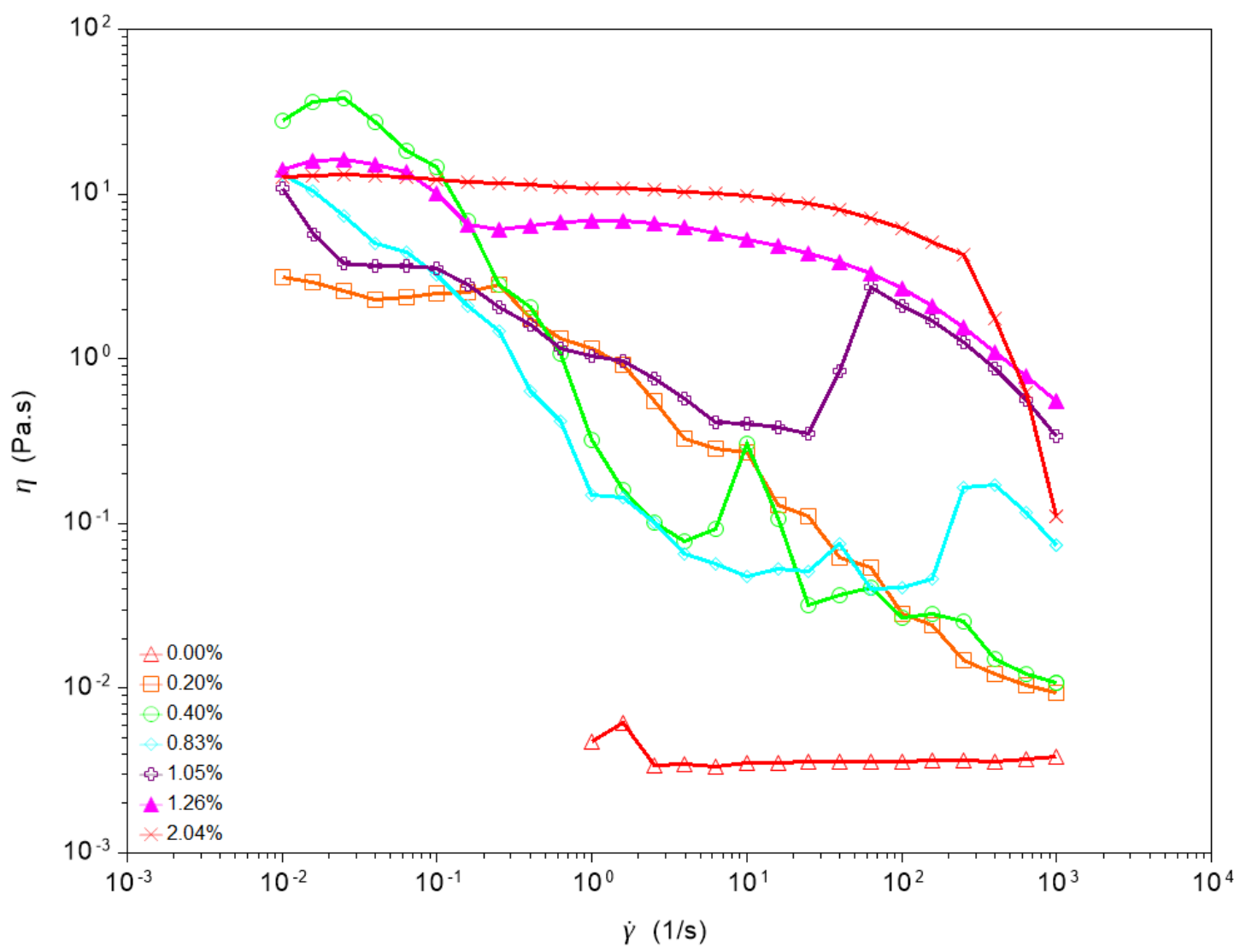

Figure 24. Viscosity Profiles of Commercial Latex / Commercial HEUR (Day 11). The Volume Fraction of Latex Was 0.25 and the Concentrations of HEUR Are Provided Inside the Figure. 


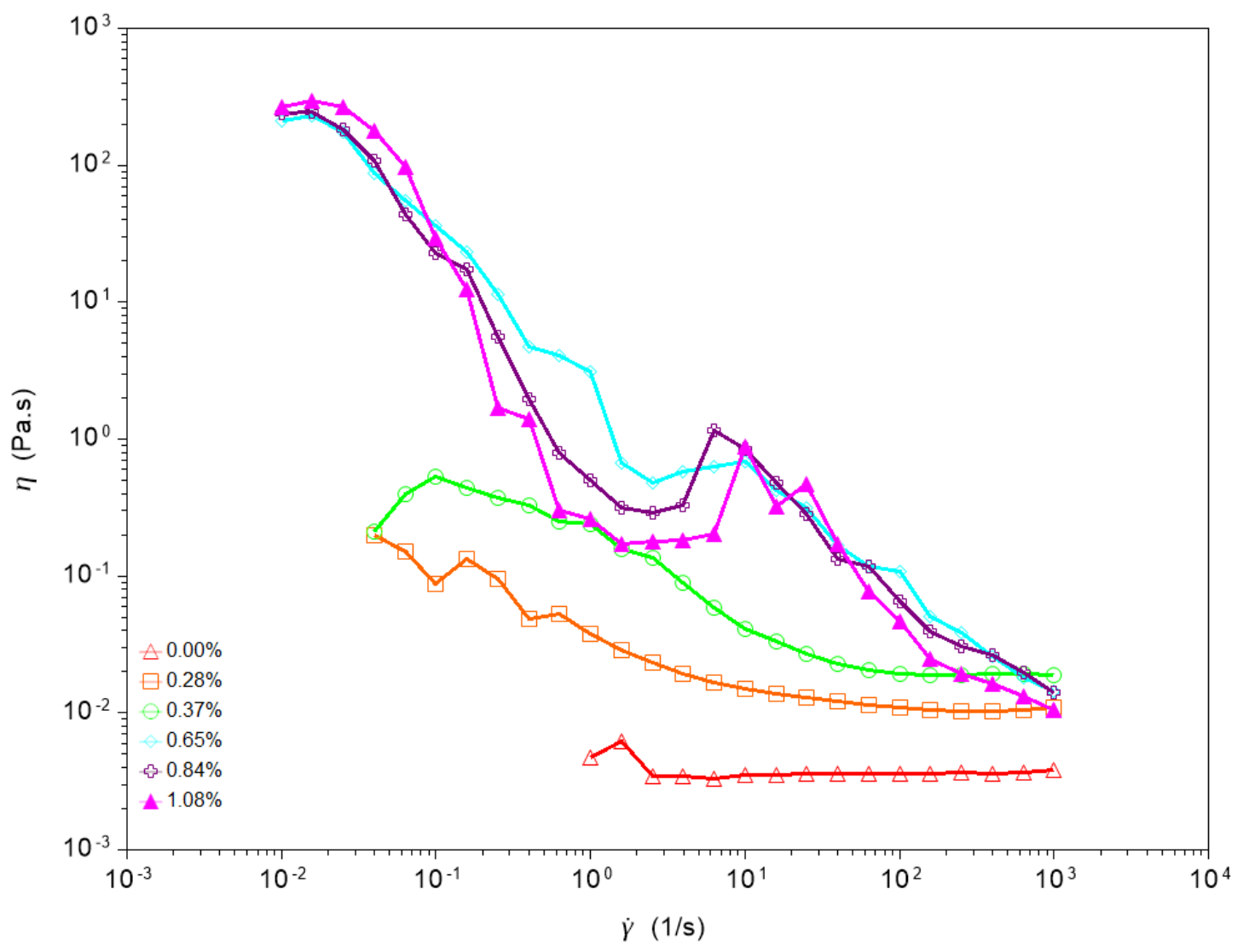

Figure 25. Viscosity Profiles of Commercial Latex / Cal Poly HEUR (Day 7). The Volume Fraction of Latex Was 0.25 and the Concentrations of HEUR Are Provided Inside the Figure. 


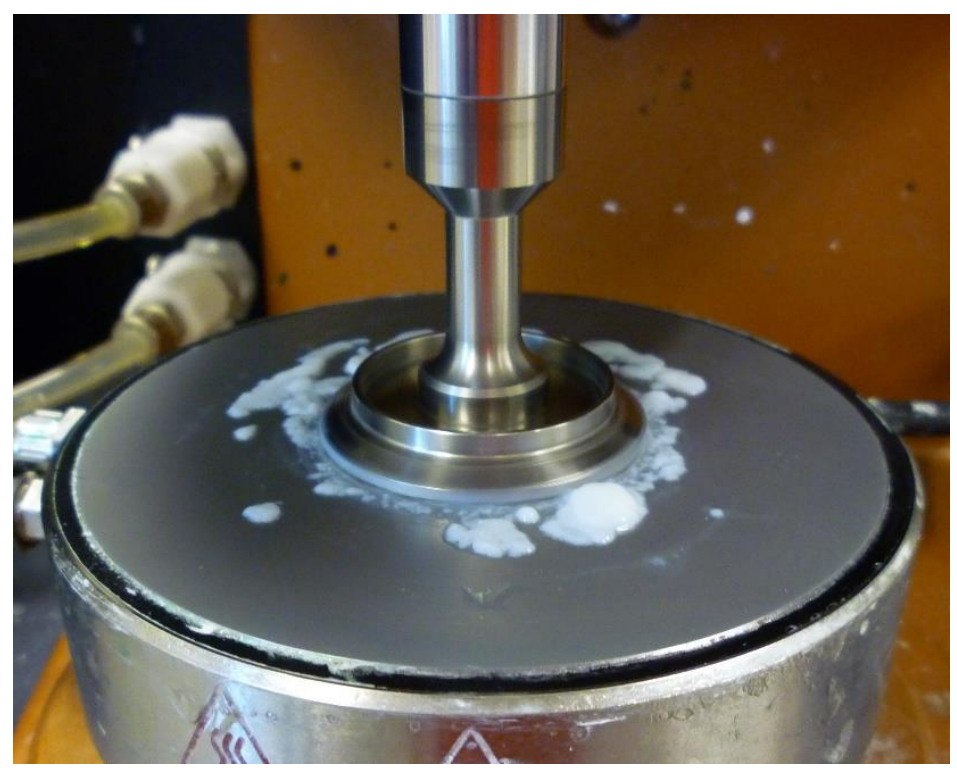

Figure 26. Ejection of Commercial Latex / Cal Poly HEUR Mixture Samples from the Cone / Plate Geometry At Within 1-100 s ${ }^{-1}$. 


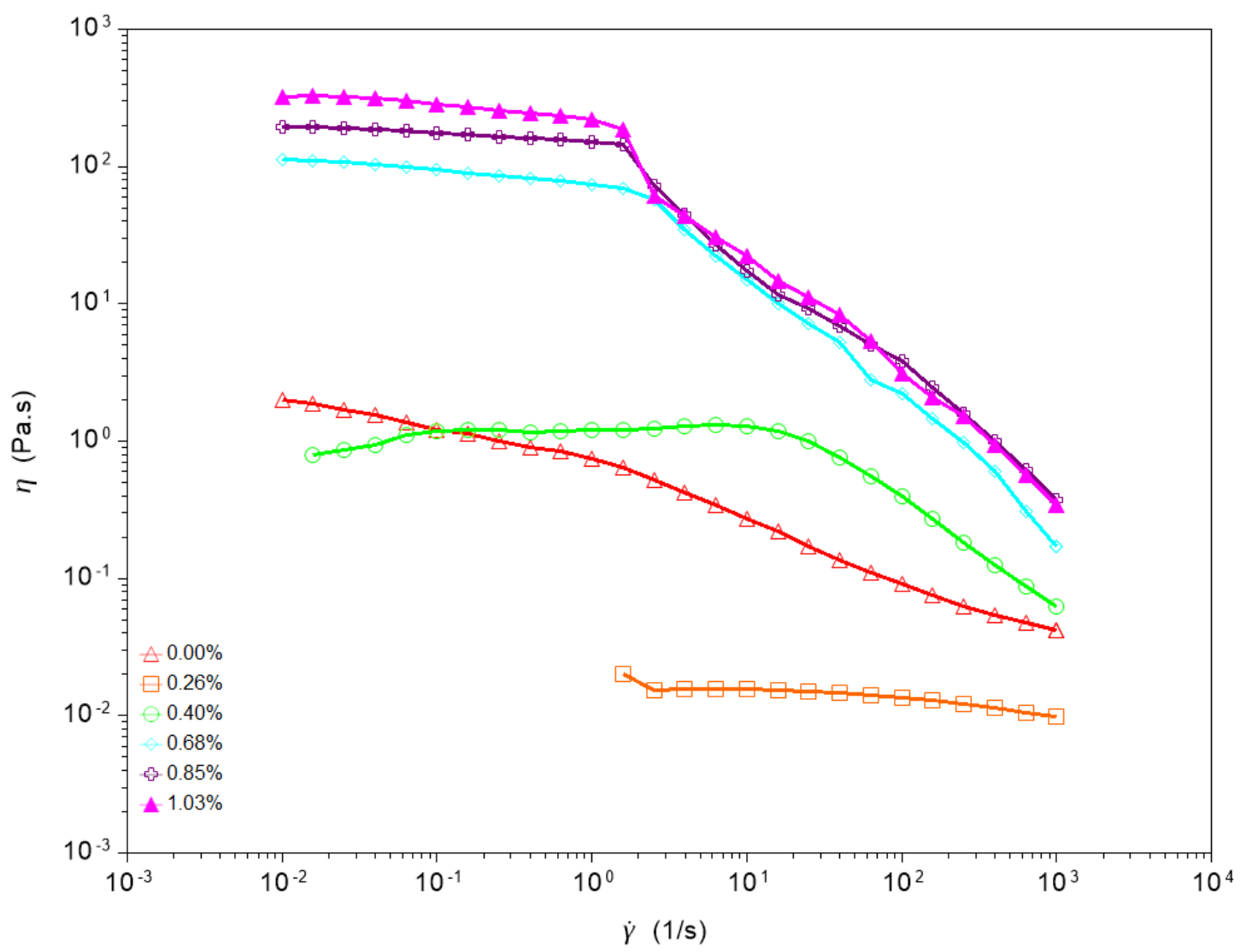

Figure 27. Viscosity Profiles of Cal Poly Latex / Cal Poly HEUR (Day 54). The Volume Fraction of Latex Was 0.30 and the Concentrations of HEUR Are Provided Inside the Figure.

\subsection{Microscopy}

Mixtures were observed by microscopy to see if there were any differences between phase-separated and dispersed mixtures. At 5x magnification, DJ-I-043 and commercial latex were featureless, with the exception of bubbles (Figure 28). The appearance of I and II samples at low HEUR concentration were irregular, though the samples transitioned to featureless appearance at HEUR concentrations at or near the HEUR concentration at which samples transitioned from phase-separated to stable dispersions. The appearance of 
the III mixtures at $0.26,0.68$, and $1.03 \mathrm{wt} \%$ HEUR were featureless, which correlates to the lack of syneresis in the III mixtures.

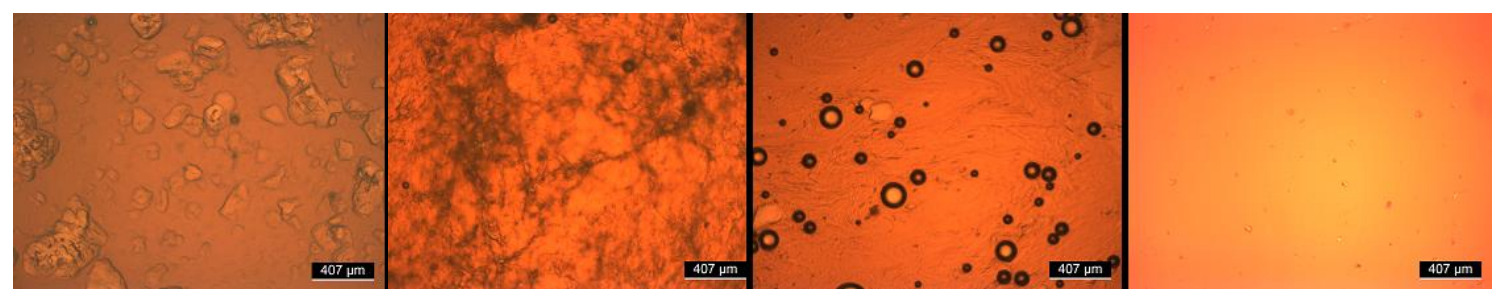

Figure 28. Transition from Irregular to Featureless Appearance in 0.10, 1.83, 2.12, and 3.19 wt\% Commercial Latex / Cal Poly HEUR Mixtures at 5x Magnification. The Black Circular Shapes in the Photographs of 1.83 and 2.12 wt\% HEUR Are Air Bubbles That Were Introduced During Sample Mixing.

\subsection{Dynamic Oscillatory Tests}

Dynamic oscillatory testing was performed to determine the relaxation times, plateau moduli, and zero-shear viscosities of I-III. In the strain sweeps of the three sets of mixtures, a strain of $1 \%$ was within the linear viscoelastic region. Thus, strain was held constant at $1 \%$ during the frequency sweeps. Only the mixtures with 0.3 and $0.8 \mathrm{wt} \%$ (I), $0.3 \mathrm{wt} \%$ (II), and $0.2 \mathrm{wt} \%$ (III) HEUR did not show linear viscoelastic behavior at $1 \%$ strain. In all of the frequency sweeps, the mixtures showed liquid-like behavior at low frequency (under $1 \mathrm{~Hz}$ ) and solid-like behavior at high frequency.

From the frequency sweeps, the suitability of the Maxwell model was determined by constructing Cole-Cole plots. The $2.1-4.2 \mathrm{wt} \%$ II produced semicircular plots that matched those calculated with eq 3 (Figure 29). For those samples both the plateau moduli and relaxation times increased with increased HEUR concentration and indicate 
enhanced associative network strength with increased HEUR concentration. A plot of zero-shear viscosity as a function of HEUR concentration suggests that the CAC of the II mixtures is $\sim 2 \mathrm{wt} \%$ HEUR.

Aside from 2.1-4.2 wt\% II, the remaining I-III did not fit the single-mode Maxwell model. However, other models, such as the multiple-mode Maxwell model, may describe these systems more adequately. 
(A)

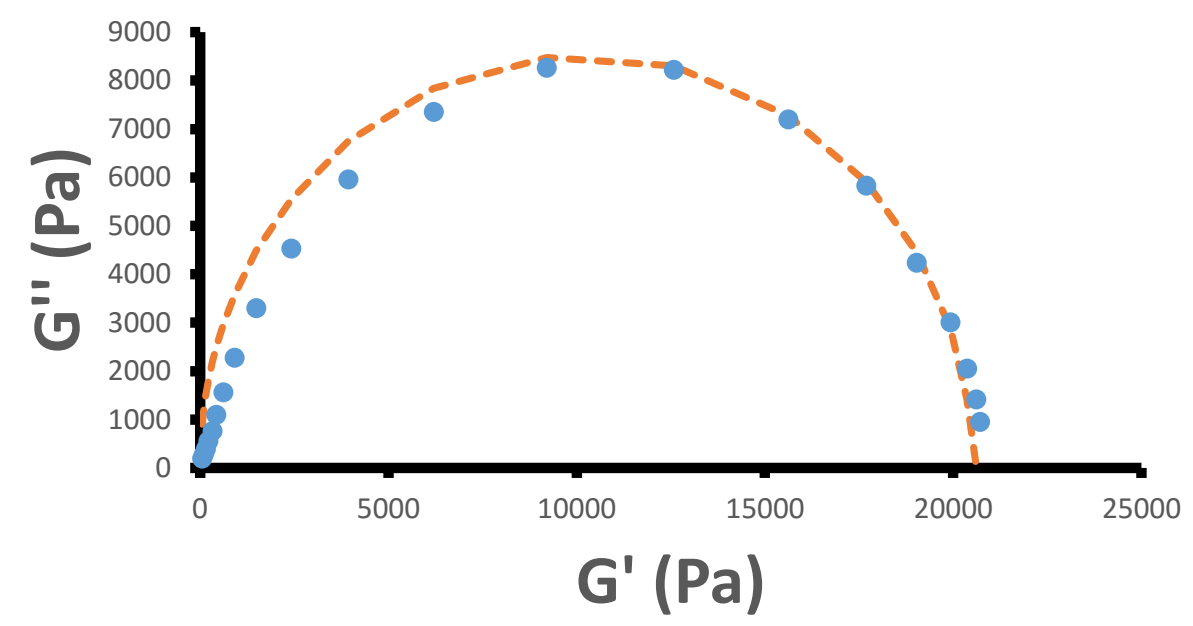

- Sample $99-\cdots \cdot$ Cole-Cole Plot

(B)

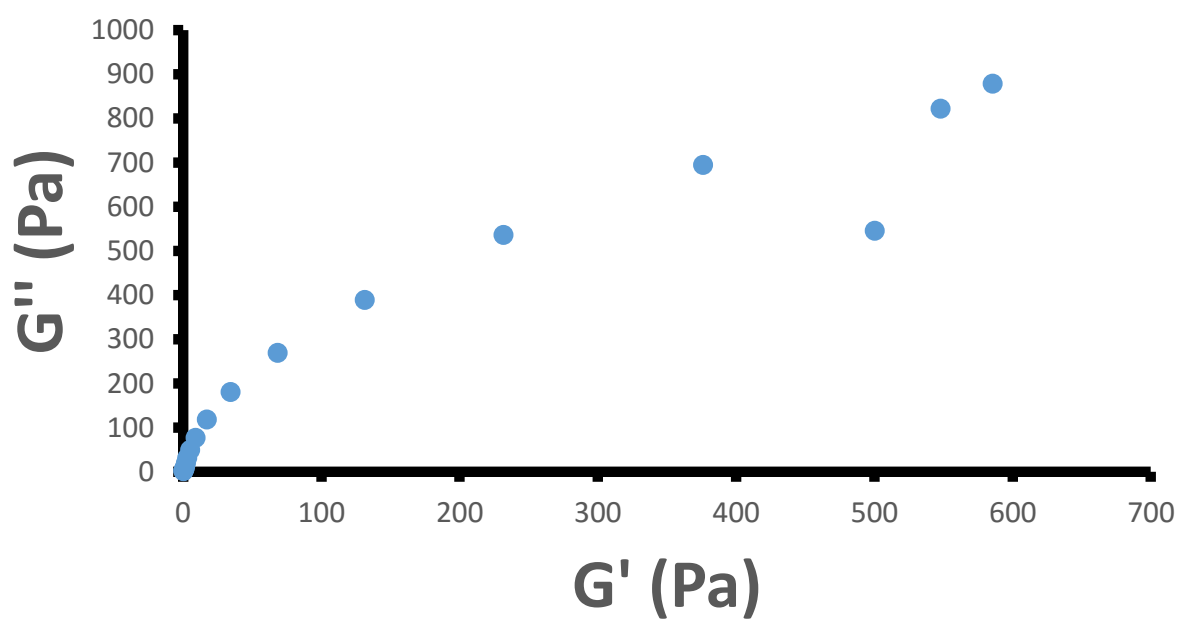

- Sample 94

Figure 29. (A) Cole-Cole Plot of 4.2 wt\% Commercial Latex / Cal Poly HEUR, Which

Fits the Single-Mode Maxwell Model; (B) Cole-Cole Plot of 1.83 wt\% Commercial

Latex / Cal Poly HEUR, Which Does Not Fit the Single-Mode Maxwell Model. 


\section{Conclusions and Future Work}

The syneresis and rheology of I-III mixtures were determined. The transition from phase-separated to stable dispersions was observable with an optical polarizable microscope at 5x magnification as a transition from irregular to featureless sample appearance. Analysis of these mixtures with the single-mode Maxwell model was limited to II mixtures with over 2 wt\% HEUR.

In agreement with the previous study at Cal Poly, phase-separated II mixtures showed complex rheology and shear thickening behavior, while dispersed III mixtures instead showed low-shear Newtonian high-shear shear thinning behavior. However, similar agreement with the dispersed II and phase-separated III mixtures is required to confirm that the suggested syneresis-rheology correlation is applicable to those mixtures. Furthermore, future studies on these latex / HEUR systems have the necessary groundwork needed to study the impact of structure and mixture composition on the complex rheology of phase-separated mixtures. This study represents progress in the ongoing study at Cal Poly to better understanding the mechanisms behind the syneresis and rheology of these latex / HEUR AT dispersions. 


\section{REFERENCES}

1. Kostansek, E. Using Dispersion / Flocculation Phase Diagrams to Visualize Interactions of Associative Polymers, Latexes, and Surfactants. Journal of Coatings Technology 2003, 75, 27-34.

2. Bell, T. J. Development and implementation of dispersion phase diagrams (DPDs) for four different hydrophobically modified ethoxylated urethane (HEUR) based acrylic paint systems. Masters' Thesis, California Polytechnic State University San Luis Obispo, San Luis Obispo, CA, 2014.

3. Saucy, D. Avoiding Viscosity Loss on Tinting. Paint \& Coatings Industry 2008, 24, 34-38.

4. Bell, T.; Fernando, R. H.; Ness, J.; Street, C.; Booth, K.; Korenkiewicz, S. Dispersion Phase Diagrams for HEUR-Thickened Acrylic Paint Systems. JCT Coatings Tech, 2015, 2, 32-39.

5. Santos, F.; Stevenson, A.; Fernando, R. H. Phase Behavior and Rheology of Latex, Associative Thickener, Surfactant Mixtures. Presented at the Western Coatings Show, Las Vegas, Nevada, 2013.

6. Schramm, G. Types of Rheometers / Viscometers. A Practical Approach to Rheology and Rheometry, Second Edition; Gebrueder HAAKE GmbH: Germany, 2000; pp 1516.

7. Wicks, Z. W.; Jones, F. N.; Pappas, S. P.; Wicks, D. A. Pigment Dispersion. Organic Coatings: Science and Technology, Third Edition; John Wiley \& Sons, Inc.: New Jersey, 2007; pp 41-62.

8. Schramm, G. Types of Rheometers / Viscometers. A Practical Approach to Rheology and Rheometry, Second Edition; Gebrueder HAAKE GmbH: Germany, 2000; pp 2129.

9. Lundberg, D. J.; Brown, R. G.; Glass, E.; Eley, R. R. Synthesis, Characterization, and Solution Rheology of Model Hydrophobically-Modified, Water-Soluble Ethoxylated Urethanes. Langmuir 1994, 10, 3027-3034.

10. Kaczmarski, J. P.; Glass, J. E. Synthesis and Solution Properties of HydrophobicallyModified Ethoxylated Urethanes with Variable Oxyethylene Spacer Lengths. Macromolecules 1993, 26, 5149-5156.

11. Barmar, M.; Kaffashi, B.; Barikani, M. Investigating the Uni-HEUR thickener performance considering hydrophilic segment length. Colloids and Surfaces A: Physiochemical and Engineering Aspects 2010, 364, 105-108. 
12. Barmar, M. Study of the Effect of PEG Length in Uni-HEUR Thickener Behavior. Journal of Applied Polymer Science, 2009, 111, 1751-1754.

13. Barmar, M.; Ribitsch, V.; Kaffashi, B.; Barikani, M.; Sarreshtehdari, Z.; Pfragner, J. Influence of prepolymers molecular weight on the viscoelastic properties of aqueous HEUR solutions. Colloid and Polymer Science, 2004, 282, 454-460.

14. Svanholm, T.; Molenaar, F.; Toussaint, A. Associative Thickeners: their adsorption behavior onto latexes and the rheology of their solutions. Progress in Organic Coatings 1997, 30, 159-165.

15. Barmar, M.; Barikani, M.; Kaffashi, B. Steady shear viscosity study of various HEUR models with different hydrophilic and hydrophobic sizes. Colloids and Surfaces A: Physiochemical and Engineering Aspects 2005, 253, 77-82.

16. Barmar, M.; Kaffashi, B.; Barikani, M. Investigating the effect of hydrophobic structural parameters on the thickening properties of HEUR associative polymers. European Polymer Journal 2005, 41, 619-626.

17. May, R.; Kaczmarski, J. P.; Glass, J. E. Influence of Molecular Weight Distributions on HEUR Aqueous Solution Rheology. Macromolecules 1996, 29, 4745-4753.

18. Kaczmarski, J. P.; Glass, J. E. Synthesis and Characterization of Step-Growth Hydrophobically-Modified Ethoxylated Urethane Associative Thickeners. Langmuir 1994, 10, 3035-3042.

19. Tanaka, F.; Edwards, S. F. Viscoelastic Properties of Physically Cross-Linked Networks: Transient Network Theory. Macromolecules 1992, 25, 1516-1523.

20. Pham, Q. T.; Russel, W. B.; Thibeault, J. C.; Lau, W. Micellar Solutions of Associative Triblock Copolymers: The Relationship between Structure and Rheology. Macromolecules 1999, 32, 5139-5146.

21. Horigome, M; Otsubo, Y. Long-Time Relaxation of Suspensions Flocculated by Associating Polymers. Langmuir 2002, 18, 1968-1973.

22. Yekta, A.; Duhamel, J.; Adiwidjaja, H.; Brochard, P.; Winnik, M. A. Association Structure of Telechelic Asssociative Thickeners in Water. Langmuir 1993, 9, 881883.

23. Annable, T.; Buscall, R.; Ettelaie, R. Network formation and its consequences for the physical behaviour of associating polymers in solution. Colloids and Surfaces A: Physicochemical and Engineering Aspects 1996, 112, 97-116.

24. Otsubo, Y. Effect of Surfactant Adsorption on the Polymer Bridging and Rheological Properties of Suspensions. Langmuir 1994, 10, 1018-1022. 
25. Tam, K. C.; Jenkins, R. D.; Winnik, M. A.; Bassett, D. R. A Structural Model of Hydrophobically Modified Urethane-Ethoxylate (HEUR) Associative Polymers in Shear Flows. Macromolecules 1998, 31, 4149-4159.

26. Jenkins, R. D. The fundamental thickening mechanism of associative polymers in latex systems: A rheological study. Ph. D. Dissertation, Lehigh University, Pennsylvania, October 1990.

27. Chen, M.; Wetzel, W. H.; Ma, Z.; Glass, J. E.; Buchacek, R. J.; Dickinson, J. G. Unifying Model for Understanding HEUR Associative Thickener Influences on Waterborne Coatings: I. HEUR Interactions with a Small Particle Latex. Journal of Coatings Technology 1997, 69, 73-80.

28. Mahli, D. M.; Steffenhagen, M. J.; Xing, L.; Glass, J. E. Surfactant Behavior and Its Influence On the Viscosity of Associative Thickeners Solutions, Thickened Latex Dispersions, and Waterborne Latex Coatings. Journal of Coatings Technology 2003, $75,39-51$.

29. Wicks, Z. W.; Jones, F. N.; Pappas, S. P.; Wicks, D. A. Pigment Dispersion. Organic Coatings: Science and Technology, Third Edition; John Wiley \& Sons, Inc.: New Jersey, 2007; pp 436-440.

30. Thibeault, J. C.; Sperry, P. R.; Schaller, E. J. Effect of Surfactants and Cosolvents on the Behavior of Associative Thickener in Latex Systems. Water-soluble polymers: Beauty with performance; Glass, J. E., Ed.; American Chemical Society, 1986; pp 375-389.

31. Schoff, C. K.; In Rheology; Brezinski, D., Miranda, T. J., Eds.; Federation Series on Coatings Technology; Federation of Societies for Coatings Technology: Pennsylvania, 1991; p 14-15.

32. Tanaka, F.; Edwards, S. F. Viscoelastic Properties of Physically Cross-Linked Networks Part 2: Dynamic mechanical moduli. Macromolecules 1992, 43, 273-288.

33. Mewis, J.; Wagner, N. J. Principles of Rheology. Colloidal Suspension Rheology; Cambridge University Press: Cambridge, 2012; pp 18-30.

34. Wu, W.; Shay, G. D. Tailoring HASE Rheology Through Polymer Design: Effects of Hydrophobe Size, Acid Content, and Molecular Weight. JCT Research 2005, 2, 423433.

35. Barmar, M.; Barikani, M.; Kaffashi, B. The effect of molecular weight on the behavior of step-growth hydrophobically modified ethoxylated urethane (S-G HEUR) end-capped with dodecyl alcohol. Iranian Polymer Journal 2004, 13, 241-246. 
36. Acrysol ${ }^{\mathrm{TM}}$ RM-825 Rheology Modifier. Rohm and Haas Company: Philadelphia, PA.

37. Rhoplex ${ }^{\mathrm{TM}}$ AC-261LF. Rohm and Haas Company: Philadelphia, PA.

38. Randall, D., Lee, S., Eds. Outline of Polyurethane Chemistry. In The Polyurethanes Book; John Wiley \& Sons, Ltd: London, 2002; pp 113-123.

39. Padfield, T.; Winslow, J.; Pedersen, W. B.; Glastrup, J. Decomposition of Polyethylene Glycol (PEG) on Heating. International Council of MuseumsConservation Committee Conference, Dresden, 1990, 243-245. 


\section{APPENDICES}

Appendix A: FTIR Spectra

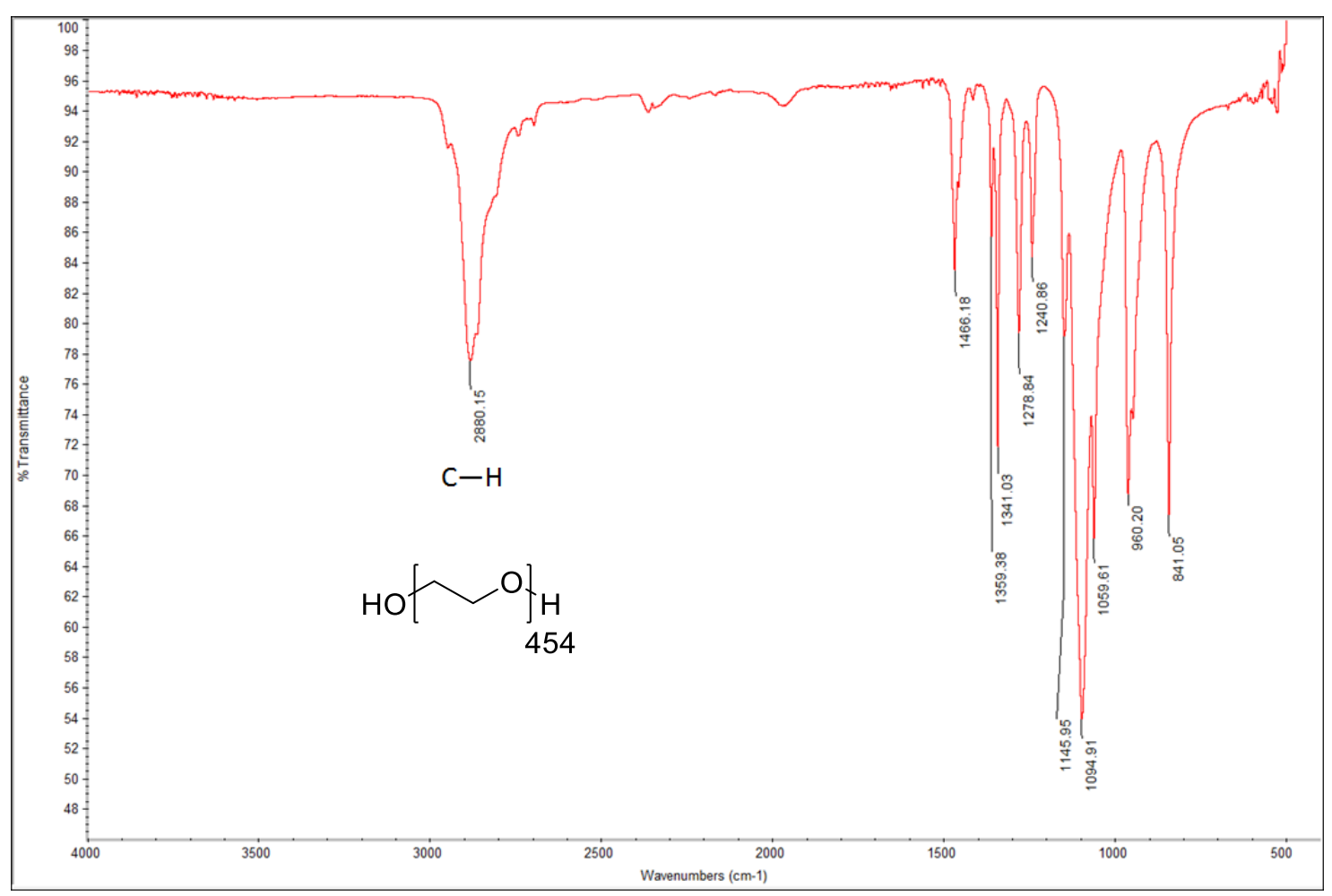

Figure 30. FTIR of PEG20. 


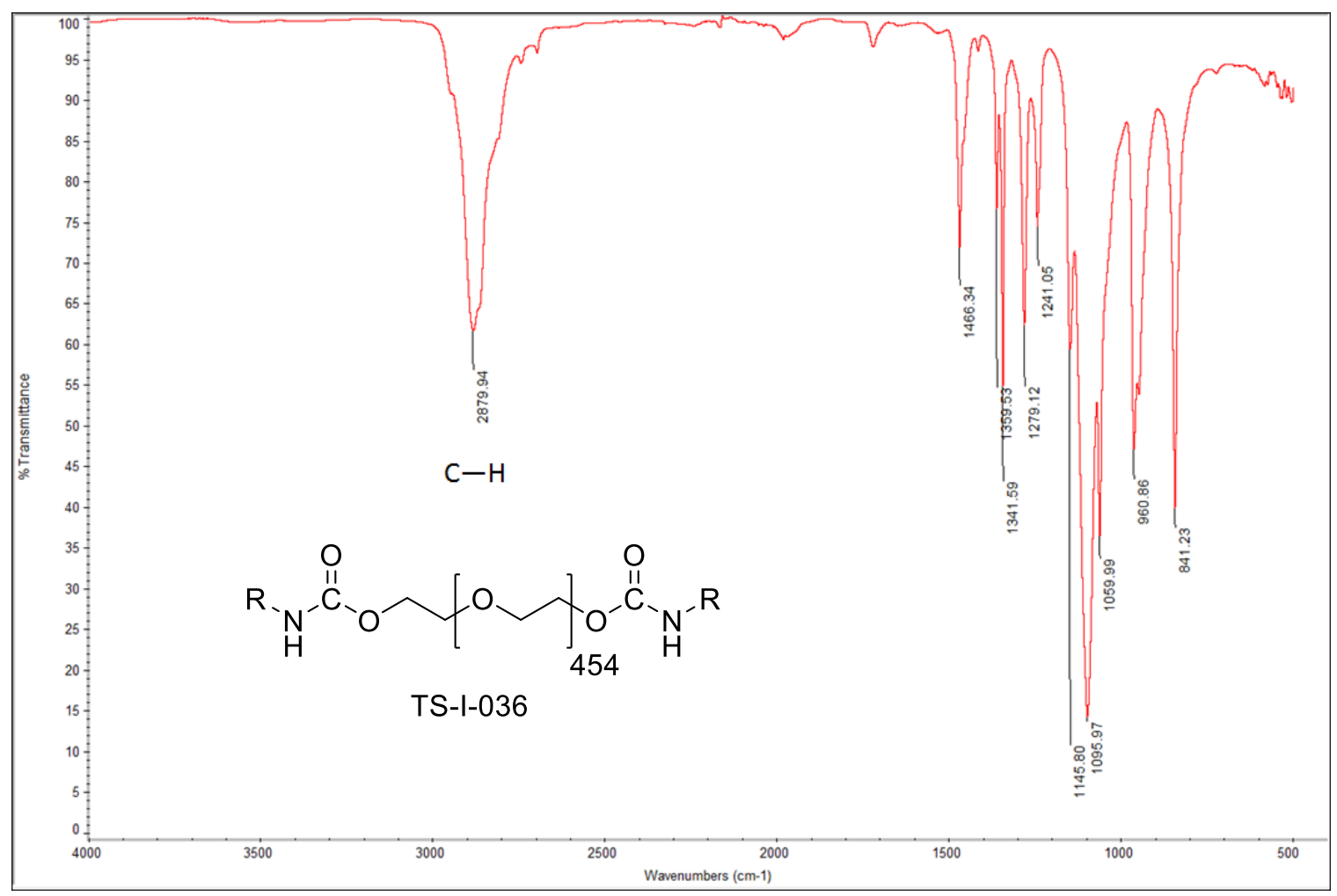

Figure 31. FTIR of TS-I-036. 


\section{Appendix B: ${ }^{1} \mathrm{H}$ NMR Spectra}

The ${ }^{1} \mathrm{H}$ NMR spectra of PEG20, TS-I-001, and TS-I-036 are presented. Due to spacing limitations on the spectra, the chemicals structures of uni-HEUR and TFAA end-capped PEG20 are provided in Figure 30. The subscript " $a$ " in $\mathrm{H}_{\mathrm{a}}$ indicates that the signal peak labelled "a" in the ${ }^{1} \mathrm{H}$ NMR spectra corresponds to the proton $\mathrm{H}_{\mathrm{a}}$, which is the terminal methylene proton of uni-HEUR.

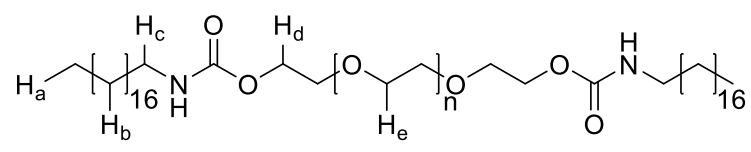<smiles>CC(COCCOC(=O)C(F)(F)F)OCC(C)OC(=O)C(F)(F)F</smiles>

Figure 32. Chemical Structures of TS-I-036 (Top) and PEG20 That Was Treated With TFAA (Bottom). The Labelled Protons Correspond to the Assigned Peaks in Figures 3336. 


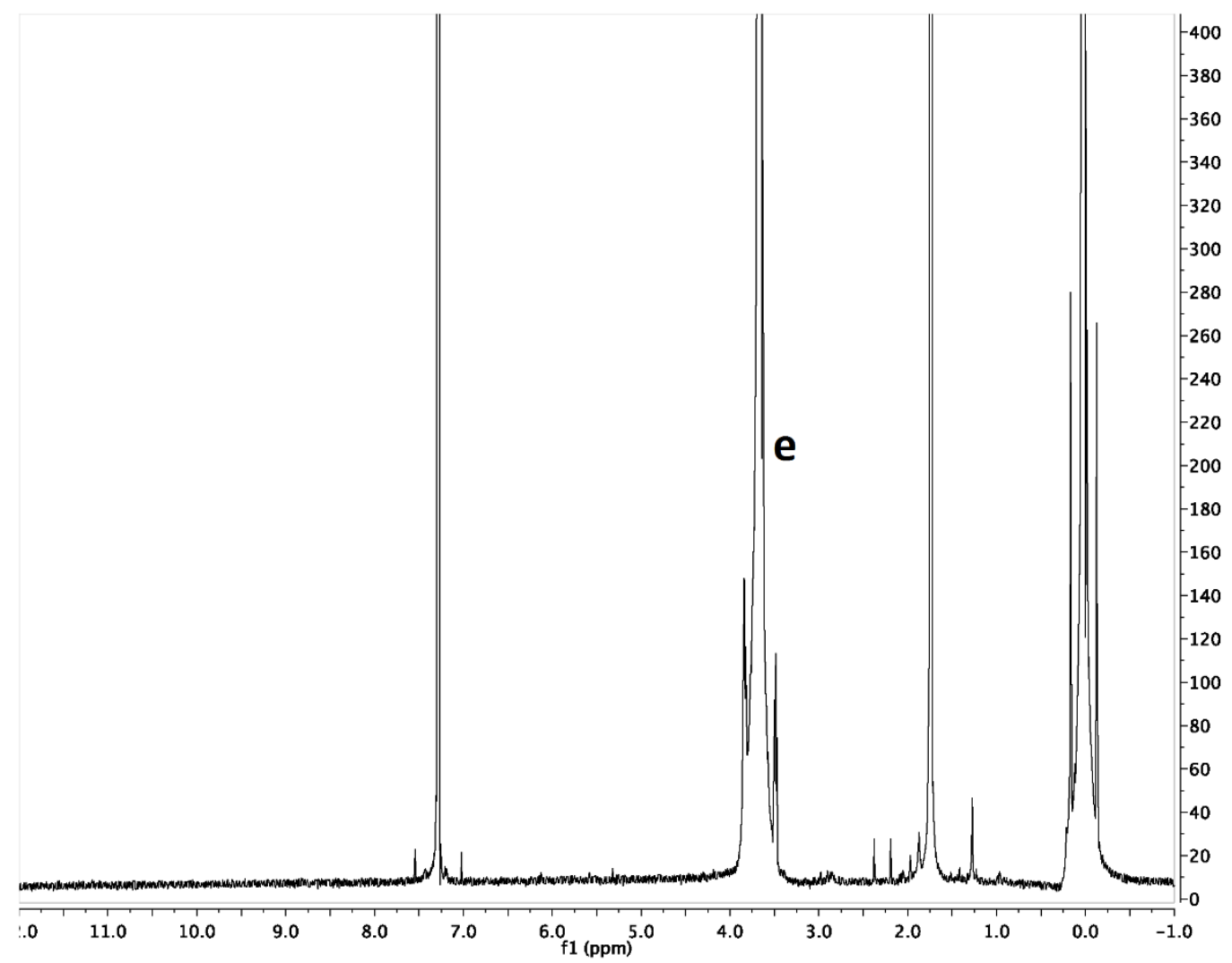

Figure 33. ${ }^{1} \mathrm{H}$ NMR of PEG20. 


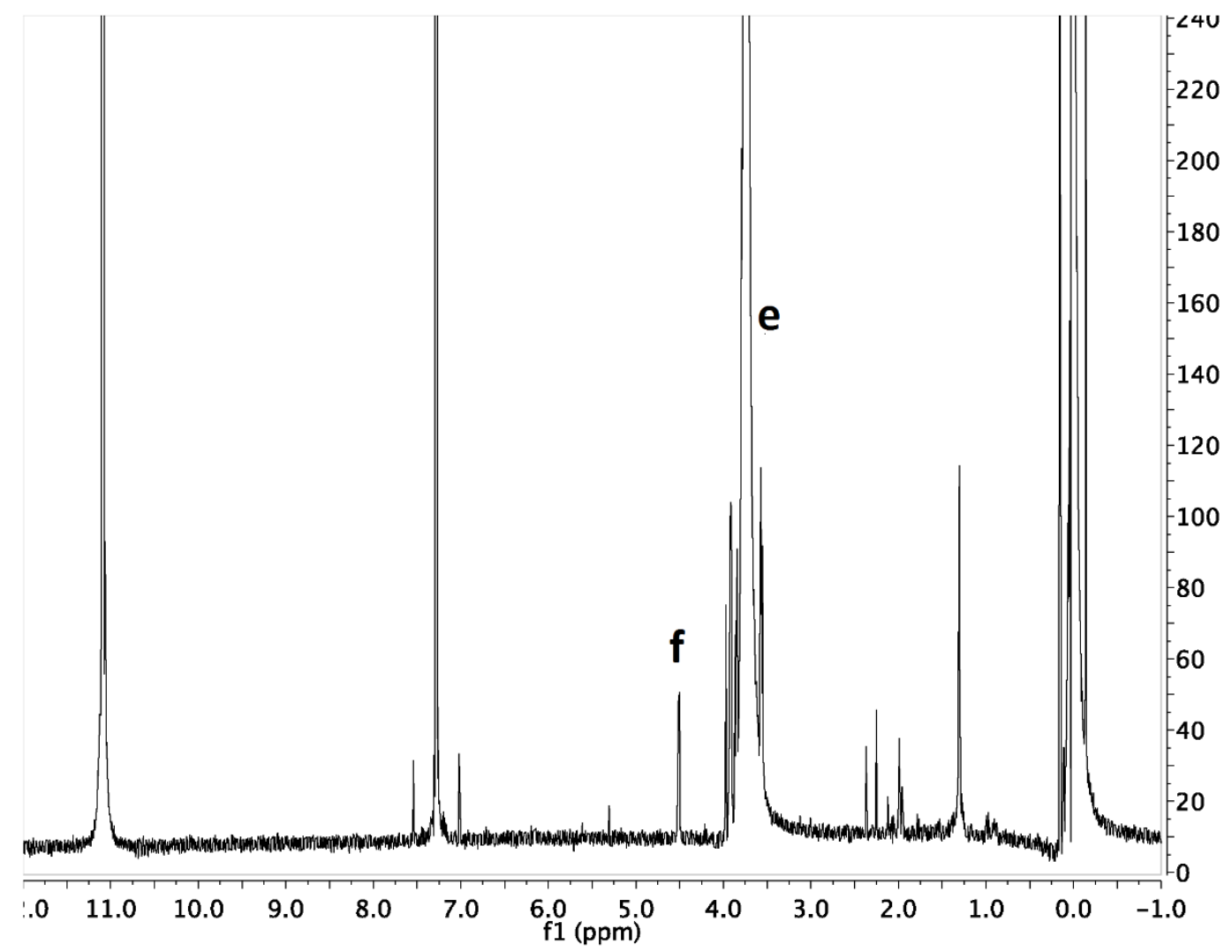

Figure 34. ${ }^{1} \mathrm{H}$ NMR of PEG20 with TFAA. 


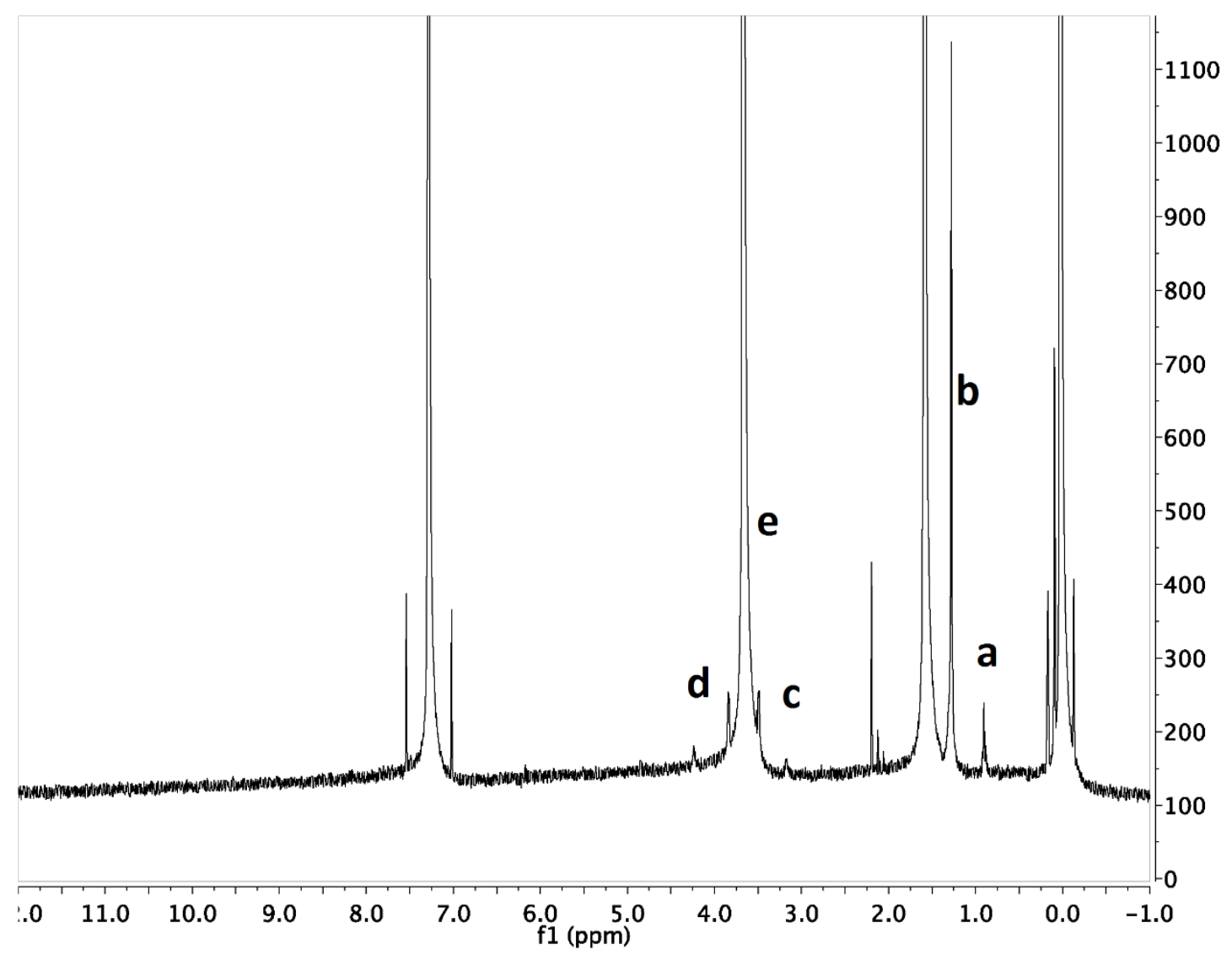

Figure 35. ${ }^{1} \mathrm{H}$ NMR of TS-I-036. 


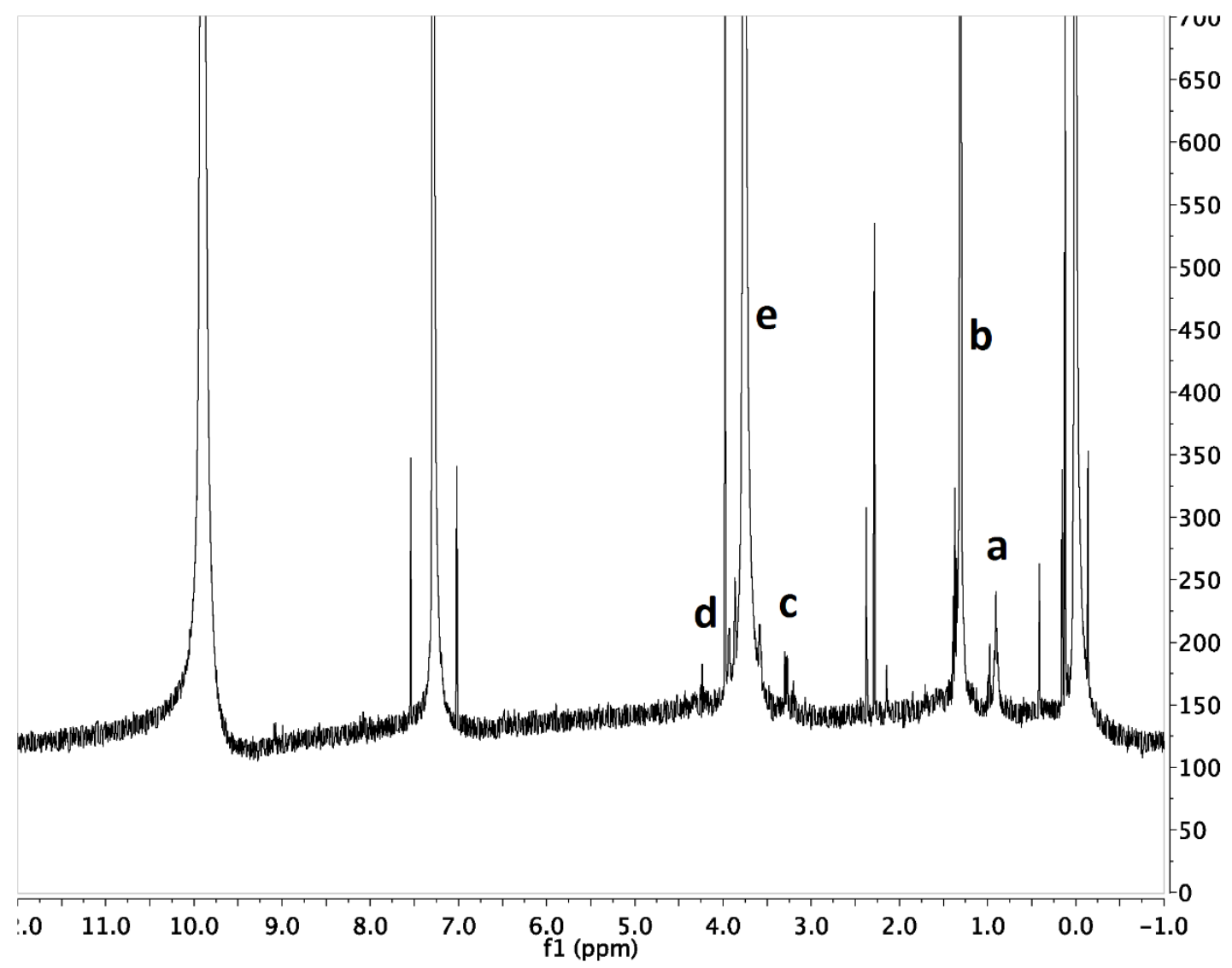

Figure 36. ${ }^{1} \mathrm{H}$ NMR of TS-I-036 with TFAA. 
Appendix C: Waterborne Latex / HEUR Mixture Compositions

Table 7. Variables That Are Used in Eqs 16-35 to Calculate the Compositions of Waterborne Latex / HEUR Mixtures.

\begin{tabular}{|l|c|c|c|c|}
\hline Variable & Mass HEUR & Mass Latex & Mass Water & Mass Acrysol \\
\hline Variable Symbol & $x$ & $y$ & $Z$ & $A$ \\
\hline Error & $0.0002 \mathrm{~g}$ & $0.002 \mathrm{~g}$ & $0.002 \mathrm{~g}$ & $0.002 \mathrm{~g}$ \\
\hline Error Symbol & $\sigma_{x}^{2}$ & $\sigma_{y}^{2}$ & $\sigma_{z}^{2}$ & $\sigma_{A}^{2}$ \\
\hline
\end{tabular}

1. For TS-I-001 and TS-I-036 mixed with latex and water...

$$
\begin{gathered}
\text { Wt } \% \text { HEUR }=\frac{x}{y+z} \times 100 \% \\
\text { Vol\% Latex }=\frac{\left(\frac{0.5 y}{1.06}\right)}{\left(\frac{y}{1.06}\right)+z} \times 100 \% \\
\text { Vol\% Water }=100 \%-\text { Vol\% Latex }
\end{gathered}
$$

Error $(\sigma)$ Analysis:

$$
\begin{gathered}
\sigma_{W t \% \text { HEUR }}=\frac{1}{y+z} \sqrt{(y+z)^{2} \sigma_{x}^{2}+x^{2}\left(\sigma_{y}^{2}+\sigma_{z}^{2}\right)} \\
\sigma_{\text {Vol\% Latex }}=\frac{B}{C^{2}} \sqrt{\left(C^{2}-\frac{2 C y}{1.06}+\frac{y^{2}}{(1.06)^{2}}\right) \sigma_{y}^{2}+y^{2} \sigma_{z}^{2}} \\
\sigma_{\text {Vol\% water }}=\sigma_{\text {Vol\% Latex }}
\end{gathered}
$$

Where...

$$
\begin{gathered}
B=\frac{0.5}{1.06} \\
C=\left(\frac{y}{1.06}+z\right)
\end{gathered}
$$


2. For waterborne commercial latex / commercial HEUR mixtures...

$$
\begin{gathered}
\text { Wt } \% \text { HEUR }=\frac{0.25 x}{0.75 x+y+z} \times 100 \% \\
\text { Vol\% Latex }=\frac{\left(\frac{0.5 y}{1.06}\right)}{(0.75 x)+\left(\frac{y}{1.06}\right)+z} \times 100 \% \\
\text { Vol\% Water }=100 \%-\text { Vol\% Latex } \\
\text { Wt\% Butyl Carbitol }=\frac{A \times 0.75 \times 0.25}{(0.75 A)+(0.5 y)+z}
\end{gathered}
$$

Error $(\sigma)$ Analysis:

$$
\begin{gathered}
\sigma_{W t \% H E U R}=\frac{1}{4 D^{2}} \sqrt{\left(D^{2}-\frac{3 D}{32}+\frac{9}{256}\right) \sigma_{x}^{2}+\sigma_{y}^{2}+\sigma_{z}^{2}} \\
\sigma_{V o l \% \text { Latex }}=\frac{B}{E^{2}} \sqrt{\frac{9 y^{2}}{16} \sigma_{x}^{2}+\left(E^{2}-\frac{2 E y}{1.06}+\frac{y^{2}}{(1.06)^{2}}\right) \sigma_{y}^{2}+y^{2} \sigma_{z}^{2}} \\
\sigma_{\text {wt\% butyl carbitol }}=\frac{A}{16 F^{2}} \sqrt{\left(\frac{9 F^{2}}{A^{2}}-\frac{27 F}{2 A}+\frac{81}{16}\right) \sigma_{A}^{2}+\frac{9}{4} \sigma_{y}^{2}+9 \sigma_{z}^{2}}
\end{gathered}
$$

Where...

$$
\begin{gathered}
B=\frac{0.5}{1.06} \\
D=0.75 x+y+z \\
E=0.75 x+\frac{1}{1.06} y+z \\
F=\frac{3}{4} A+\frac{1}{2} y+z
\end{gathered}
$$


Table 8. Compositions of the Commercial Latex / Commercial HEUR Mixtures.

\begin{tabular}{c|cccc|ccc} 
Sample & $\begin{array}{c}\text { HEUR } \\
\text { (wt\%) }\end{array}$ & $\begin{array}{c}\text { Latex } \\
\text { (vol\%) }\end{array}$ & $\begin{array}{c}\text { H2O } \\
\text { (vol\%) }\end{array}$ & $\begin{array}{c}\text { Butyl } \\
\text { Carbitol } \\
\text { (wt\%) }\end{array}$ & HEUR & Latex & $\begin{array}{c}\text { Error Values } \\
\text { Carbitol }\end{array}$ \\
\hline $\mathbf{8 1}$ & $0.10 \%$ & $23.95 \%$ & $76.05 \%$ & $0.03 \%$ & $0.0002 \%$ & $0.0003 \%$ & $0.0002 \%$ \\
$\mathbf{8 2}$ & $0.20 \%$ & $24.10 \%$ & $75.90 \%$ & $0.05 \%$ & $0.0002 \%$ & $0.0003 \%$ & $0.0002 \%$ \\
$\mathbf{8 3}$ & $0.30 \%$ & $24.27 \%$ & $75.73 \%$ & $0.08 \%$ & $0.0002 \%$ & $0.0003 \%$ & $0.0002 \%$ \\
$\mathbf{8 4}$ & $0.40 \%$ & $24.42 \%$ & $75.58 \%$ & $0.11 \%$ & $0.0002 \%$ & $0.0003 \%$ & $0.0002 \%$ \\
$\mathbf{8 5}$ & $0.51 \%$ & $24.37 \%$ & $75.63 \%$ & $0.15 \%$ & $0.0002 \%$ & $0.0003 \%$ & $0.0002 \%$ \\
$\mathbf{8 6}$ & $0.62 \%$ & $24.49 \%$ & $75.51 \%$ & $0.19 \%$ & $0.0002 \%$ & $0.0003 \%$ & $0.0002 \%$ \\
$\mathbf{8 7}$ & $0.73 \%$ & $24.67 \%$ & $75.33 \%$ & $0.23 \%$ & $0.0002 \%$ & $0.0003 \%$ & $0.0002 \%$ \\
$\mathbf{8 8}$ & $0.83 \%$ & $24.70 \%$ & $75.30 \%$ & $0.27 \%$ & $0.0002 \%$ & $0.0004 \%$ & $0.0002 \%$ \\
$\mathbf{8 9}$ & $0.94 \%$ & $24.87 \%$ & $75.13 \%$ & $0.32 \%$ & $0.0002 \%$ & $0.0004 \%$ & $0.0002 \%$ \\
$\mathbf{9 0}$ & $1.05 \%$ & $24.92 \%$ & $75.08 \%$ & $0.37 \%$ & $0.0002 \%$ & $0.0004 \%$ & $0.0003 \%$ \\
$\mathbf{9 1}$ & $1.26 \%$ & $25.19 \%$ & $74.81 \%$ & $0.49 \%$ & $0.0002 \%$ & $0.0004 \%$ & $0.0003 \%$ \\
$\mathbf{9 2}$ & $1.48 \%$ & $25.33 \%$ & $74.67 \%$ & $0.64 \%$ & $0.0003 \%$ & $0.0005 \%$ & $0.0003 \%$ \\
$\mathbf{9 3}$ & $1.71 \%$ & $25.60 \%$ & $74.40 \%$ & $0.83 \%$ & $0.0003 \%$ & $0.0006 \%$ & $0.0003 \%$ \\
$\mathbf{9 4}$ & $1.94 \%$ & $25.82 \%$ & $74.18 \%$ & $1.07 \%$ & $0.0003 \%$ & $0.0007 \%$ & $0.0004 \%$ \\
$\mathbf{9 5}$ & $2.04 \%$ & $24.49 \%$ & $75.51 \%$ & $1.41 \%$ & $0.0003 \%$ & $0.0008 \%$ & $0.0005 \%$
\end{tabular}


Table 9. Compositions of the Commercial Latex / Cal Poly HEUR Mixtures.

\begin{tabular}{c|ccc|cc} 
Sample & HEUR & Latex & H20 & Error Values \\
& (wt\%) & $\mathbf{( v o l \% )}$ & $\mathbf{( v 0 l \% )}$ & HEUR & Latex \\
\hline 48 & $0.19 \%$ & $24.36 \%$ & $75.64 \%$ & $0.02 \%$ & $0.0002 \%$ \\
49 & $0.30 \%$ & $23.96 \%$ & $76.04 \%$ & $0.02 \%$ & $0.0002 \%$ \\
50 & $0.39 \%$ & $23.92 \%$ & $76.08 \%$ & $0.02 \%$ & $0.0002 \%$ \\
51 & $0.47 \%$ & $24.63 \%$ & $75.37 \%$ & $0.02 \%$ & $0.0002 \%$ \\
52 & $0.58 \%$ & $24.05 \%$ & $75.95 \%$ & $0.02 \%$ & $0.0002 \%$ \\
53 & $0.74 \%$ & $24.10 \%$ & $75.90 \%$ & $0.02 \%$ & $0.0002 \%$ \\
54 & $0.79 \%$ & $24.15 \%$ & $75.85 \%$ & $0.02 \%$ & $0.0002 \%$ \\
55 & $0.89 \%$ & $24.10 \%$ & $75.90 \%$ & $0.02 \%$ & $0.0002 \%$ \\
56 & $0.97 \%$ & $24.21 \%$ & $75.79 \%$ & $0.02 \%$ & $0.0002 \%$ \\
57 & $1.20 \%$ & $23.79 \%$ & $76.21 \%$ & $0.02 \%$ & $0.0002 \%$ \\
58 & $1.41 \%$ & $24.30 \%$ & $75.70 \%$ & $0.02 \%$ & $0.0002 \%$ \\
59 & $1.68 \%$ & $24.04 \%$ & $75.96 \%$ & $0.02 \%$ & $0.0002 \%$ \\
60 & $1.82 \%$ & $23.89 \%$ & $76.11 \%$ & $0.02 \%$ & $0.0002 \%$ \\
61 & $2.03 \%$ & $24.09 \%$ & $75.91 \%$ & $0.02 \%$ & $0.0002 \%$ \\
62 & $0.20 \%$ & $23.93 \%$ & $76.07 \%$ & $0.02 \%$ & $0.0003 \%$ \\
63 & $0.28 \%$ & $23.96 \%$ & $76.04 \%$ & $0.02 \%$ & $0.0003 \%$ \\
64 & $0.37 \%$ & $23.58 \%$ & $76.42 \%$ & $0.02 \%$ & $0.0003 \%$ \\
65 & $0.47 \%$ & $24.01 \%$ & $75.99 \%$ & $0.02 \%$ & $0.0003 \%$ \\
66 & $0.57 \%$ & $23.95 \%$ & $76.05 \%$ & $0.02 \%$ & $0.0003 \%$ \\
67 & $0.65 \%$ & $24.09 \%$ & $75.91 \%$ & $0.02 \%$ & $0.0003 \%$ \\
68 & $0.74 \%$ & $24.14 \%$ & $75.86 \%$ & $0.02 \%$ & $0.0003 \%$ \\
69 & $0.84 \%$ & $24.09 \%$ & $75.91 \%$ & $0.02 \%$ & $0.0003 \%$ \\
70 & $0.94 \%$ & $24.03 \%$ & $75.97 \%$ & $0.02 \%$ & $0.0003 \%$ \\
71 & $1.08 \%$ & $24.09 \%$ & $75.91 \%$ & $0.02 \%$ & $0.0004 \%$ \\
72 & $1.28 \%$ & $23.92 \%$ & $76.08 \%$ & $0.02 \%$ & $0.0004 \%$ \\
73 & $1.46 \%$ & $24.02 \%$ & $75.98 \%$ & $0.02 \%$ & $0.0005 \%$ \\
74 & $1.66 \%$ & $24.22 \%$ & $75.78 \%$ & $0.02 \%$ & $0.0006 \%$ \\
75 & $1.83 \%$ & $24.13 \%$ & $75.87 \%$ & $0.02 \%$ & $0.0007 \%$ \\
76 & $0.10 \%$ & $23.47 \%$ & $76.53 \%$ & $0.02 \%$ & $0.0002 \%$ \\
77 & $0.30 \%$ & $24.04 \%$ & $75.96 \%$ & $0.02 \%$ & $0.0003 \%$ \\
78 & $0.33 \%$ & $24.09 \%$ & $75.91 \%$ & $0.02 \%$ & $0.0003 \%$ \\
79 & $0.38 \%$ & $24.14 \%$ & $75.86 \%$ & $0.02 \%$ & $0.0003 \%$ \\
80 & $0.36 \%$ & $24.07 \%$ & $75.93 \%$ & $0.02 \%$ & $0.0003 \%$ \\
96 & $2.12 \%$ & $24.44 \%$ & $75.56 \%$ & $0.02 \%$ & $0.0003 \%$ \\
97 & $3.19 \%$ & $24.21 \%$ & $75.79 \%$ & $0.02 \%$ & $0.0005 \%$ \\
98 & $3.61 \%$ & $24.80 \%$ & $75.20 \%$ & $0.02 \%$ & $0.0006 \%$ \\
99 & $4.19 \%$ & $24.70 \%$ & $75.30 \%$ & $0.02 \%$ & $0.0007 \%$
\end{tabular}


Table 10. Compositions of the Cal Poly Latex / Cal Poly HEUR Mixtures.

\begin{tabular}{c|ccc|cc} 
Sample & HEUR & Latex & H2O & \multicolumn{2}{c}{ Error Values } \\
& $(\mathbf{w t \% )}$ & $(\mathbf{v o l \% )}$ & $(\mathbf{v o l \% )}$ & HEUR & Latex \\
\hline 43 & $0.26 \%$ & $29.27 \%$ & $70.73 \%$ & $0.02 \%$ & $0.0004 \%$ \\
44 & $0.40 \%$ & $29.08 \%$ & $70.92 \%$ & $0.02 \%$ & $0.0004 \%$ \\
45 & $0.68 \%$ & $28.93 \%$ & $71.07 \%$ & $0.02 \%$ & $0.0004 \%$ \\
46 & $0.85 \%$ & $29.08 \%$ & $70.92 \%$ & $0.02 \%$ & $0.0004 \%$ \\
47 & $1.03 \%$ & $29.01 \%$ & $70.99 \%$ & $0.02 \%$ & $0.0004 \%$
\end{tabular}




\section{Appendix D: Determination of Percent Syneresis Using ImageJ}

A set of mixtures was placed in a Byko-spectra light book under "Store" light and a picture taken with a hand-held Panasonic DMC-FS15 camera. Using ImageJ software, a public domain Java image processing program, a straight yellow line was drawn from slightly above the same to below the 11-dram vial (Figure 37). Black paper was placed behind the samples to enhance the contrast between the top and bottom layers of the dispersion.

A plot profile of the image was then generated (Figure 38), which plots the blackwhite value of the image along the yellow line, traveling from the top of the image (above the aqueous layer) to the bottom (the white paper below the 11-dram vial). The percent syneresis was determined from such a plot using the pixel values of the three different regions that are outlined in Figure 38. The first region marks the top of the aqueous layer, where the image becomes blacker as the cursor travels down the image. The second region marks the bottom of the aqueous layer and top of the latex layer, where the image becomes whiter. The third and final region marks the bottom of the latex layer (and bottom of the vial), where the image sharply transitions between two whitecolored regions. For example, the pixel values for sample 73 (day 31) determined from Figure 38 are 220,424, and 1180. With those values, the percent syneresis was calculated from the ratio of layer heights (eq 36 ). For sample 73 , the percent syneresis $(S$ ) is $21.3 \%$.

$$
\frac{S}{100 \%}=\frac{\text { region } 2-\text { region } 1}{\text { region } 3-\text { region } 1}=\frac{y-x}{z-x}
$$


The error of the resultant value was determined from eq 37, where the pixel values of regions $1-3$ were denoted $x, y$, and $z$, respectively, and the error of a value was denoted with the subscript $e$.

$$
S_{e}=\sqrt{\left(\frac{\partial S}{\partial x}\right)^{2} x_{e}^{2}+\left(\frac{\partial S}{\partial y}\right)^{2} y_{e}^{2}+\left(\frac{\partial S}{\partial z}\right)^{2} z_{e}^{2}} \times 100 \%
$$

The error of each measurement was estimated as \pm 20 pixels $\left(x_{e}=y_{e}=z_{e}=20\right.$ pixels $)$, a value that was based on how the pixel value changes when the cursor is shifted slightly to the left or right. When all three pixel values are shifted by $x$ pixels (such that $a=0$, $b=y-x$, and $c=z-x)$, eq 37 is reduced to eq 38 when the derivatives are evaluated.

$$
S_{e}=\frac{20}{c^{2}} \sqrt{b^{2}+c^{2}} \times 100 \%
$$

Since syneresis varied from 5-50\% among all dispersions and the height of the dispersions from 750-1250 pixels, $S_{e}$ varied from 1.6-3.0\% (Table 11).

Table 11. Error of the Calculated Percent Syneresis Values (as a Percent) of the Waterborne Latex / HEUR Mixtures as the Size of the Top and Bottom Layers Varies.

\begin{tabular}{c|ccc} 
& \multicolumn{3}{c}{$c$ (pixels) } \\
$S(\%)$ & 750 & 1000 & 1250 \\
\hline 5 & 2.67 & 2.00 & 1.60 \\
25 & 2.75 & 2.06 & 1.65 \\
50 & 2.98 & 2.24 & 1.79
\end{tabular}




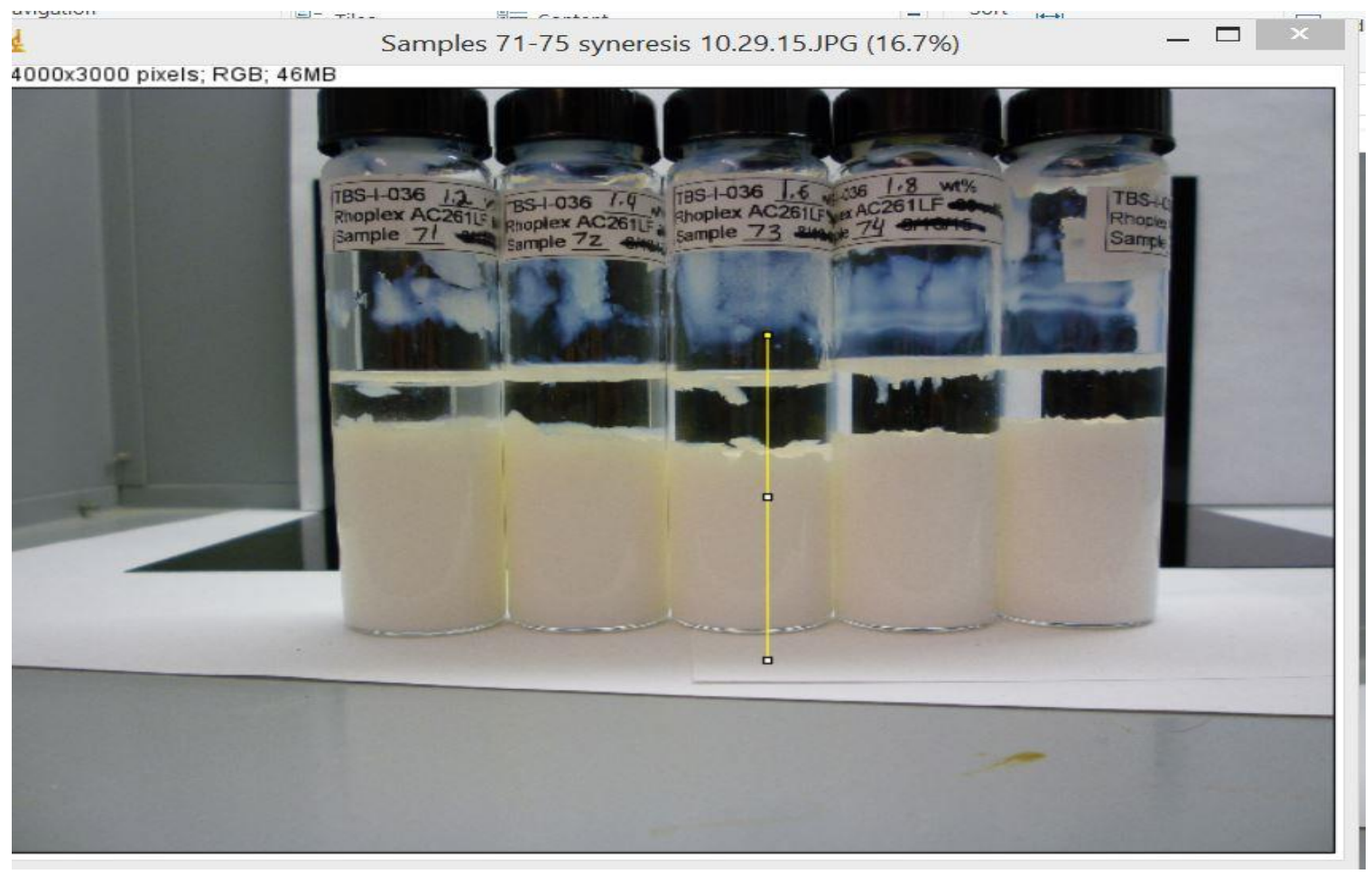

Figure 37. Yellow-line Drawn Across the Sample 73 (Day 32) using ImageJ. 


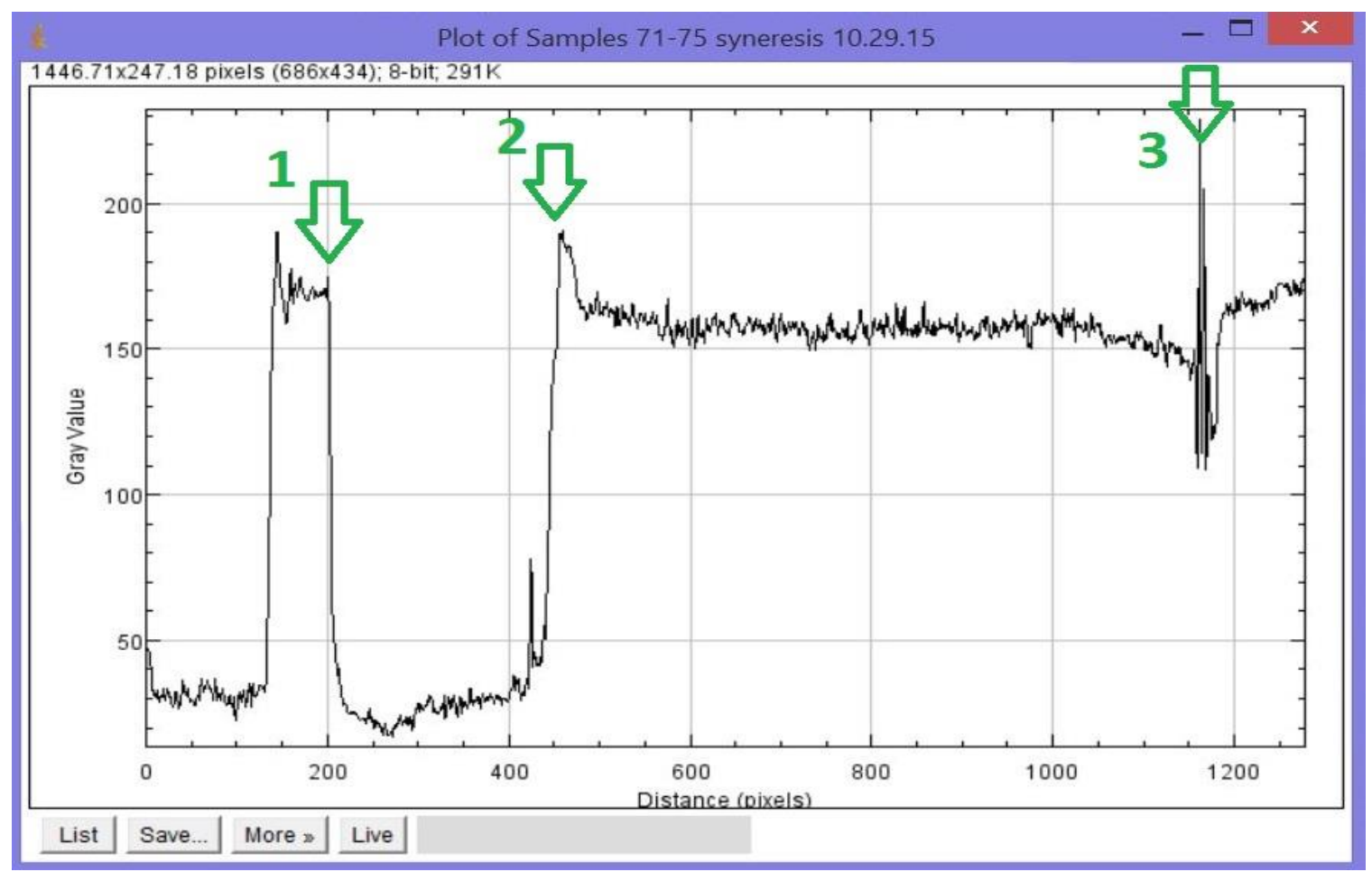

Figure 38. Plot Profile of Sample 73 (day 32), Obtained with ImageJ. The Arrows Correspond to the Pixel Values that Mark the Boundaries of the Top and Bottom Layers of the Waterborne Latex / HEUR Mixture. 